\title{
Assessing the match performance of non-ideal operational facial images using 3D image data.
}

\author{
Rupindrani Aila
}

Follow this and additional works at: https://researchrepository.wvu.edu/etd

\section{Recommended Citation}

Aila, Rupindrani, "Assessing the match performance of non-ideal operational facial images using 3D image data." (2016). Graduate Theses, Dissertations, and Problem Reports. 3994.

https://researchrepository.wvu.edu/etd/3994

This Problem/Project Report is protected by copyright and/or related rights. It has been brought to you by the The Research Repository @WVU with permission from the rights-holder(s). You are free to use this Problem/Project Report in any way that is permitted by the copyright and related rights legislation that applies to your use. For other uses you must obtain permission from the rights-holder(s) directly, unless additional rights are indicated by a Creative Commons license in the record and/ or on the work itself. This Problem/Project Report has been accepted for inclusion in WVU Graduate Theses, Dissertations, and Problem Reports collection by an authorized administrator of The Research Repository @ WVU. For more information, please contact researchrepository@mail.wvu.edu. 


\title{
Assessing the Match Performance of Non- Ideal Operational Facial Images Using 3D Image Data
}

\author{
by \\ Rupindrani Aila \\ Master's Problem report submitted to the \\ Benjamin M. Statler College of Engineering and Mineral Resources \\ At \\ West Virginia University \\ in partial fulfillment of the requirements \\ for the degree, of \\ Master of Science \\ in \\ Electrical Engineering \\ Dr. Jeremy M. Dawson, Ph. D., Chair \\ Dr. Thirimachos Bourlai, Ph. D \\ Dr. Xin Li, Ph. D \\ Lane Department of Computer Science and Electrical Engineering
}

Morgantown, West Virginia

2016

Keywords: Face Recognition, Pose-Varying, Pose-Normalization Copyright 2016 Rupindrani Aila 


\section{Abstract}

\section{Assessing the Match Performance of Non-Ideal Operational Facial Images Using 3D Image Data}

By

\section{Rupindrani Aila}

Biometric attributes are unique characteristics specific to an individual, which can be used in automated identification schemes. There have been considerable advancements in the field of face recognition recently, but challenges still exist. One of these challenges is pose-variation, specifically, roll, pitch, and yaw variations away from a frontal image. The goal of this problem report is to assess the improvement of facial recognition performance obtainable by commercial pose-correction software. This was done using pose-corrected images obtained in two ways: 1) non-frontal images generated and corrected using 3D facial scans (pseudo-pose-correction) and 2) the same non-frontal images corrected using FaceVACs DBScan. Two matchers were used to evaluate matching performance namely Cognitec FaceVACs and MegaMatcher 5.0 SDK. A set of matching experiments were conducted using frontal, non-frontal and pose-corrected images to assess the improvement in matching performance, including: 1. Frontal (probe) to Frontal (gallery) images, to generate the baseline 2. Non-ideal pose-varying (probe) to frontal (gallery) 3. Pseudo-pose-corrected (probe) to frontal (gallery) 4. Auto-pose-corrected (probe) to frontal (gallery). Cumulative match characteristics curves (CMC) are used to evaluate the performance of the match scores generated. These matching results have shown better performance in case of pseudo-pose-corrected images compared to the nonfrontal images, where the rank accuracy is $100 \%$ for the angles which were not detected by the matchers in the non-frontal case. Of the two commercial matchers, Cognitec, which is software optimized for non-frontal models, has shown a better performance in detection of face with angular rotations. MegaMatcher, which is not a pose-correction matcher, was unable to detect greater angles of rotation which are $50^{\circ}$ and $60^{\circ}$ in pitch, greater than $40^{\circ}$ for yaw and for coupled pitch/yaw it was unable to detect 4 out of 8 combinations. The requirements of the facial recognition application will influence the decision to implement pose correction tools. 


\section{Acknowledgements}

First and foremost, I would like express my profound gratitude to my advisor Dr. Jeremy Dawson for providing me an opportunity for research and for his constant support and guidance throughout my masters without whom I could not have made it this long. I would also like to thank Dr. Thirimachos Bourlai, and Dr. Xin Li for accepting to serve on my committee.

I owe my sincere thanks and dedicate this thesis to my mother Aila Vimala Devi, for her support and encouragement. She has been my pillar of support and strength at all the times, without whom this would not have been possible.

I am very much indebted to Raghu Nandan Pasula and my brother Chandra Shekar for their valuable help throughout the work. Special thanks to my friends Lahari Vazrala, Loukhya Kakamanu, Monica Kamireddy, Nikitha Nadiminti and Ajay Krishna Teja for their enormous support.

Above all, I thank lord almighty for everything in my life. 


\section{Table of Contents}

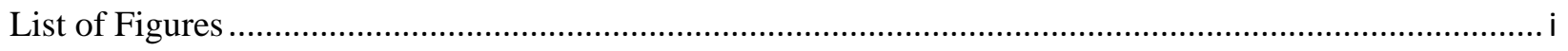

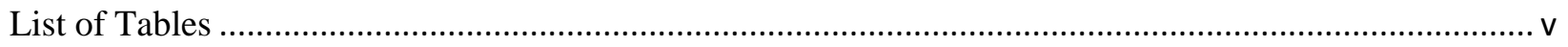

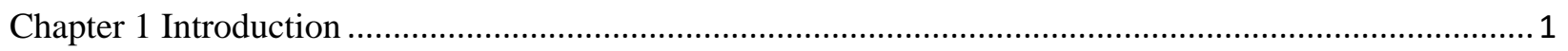

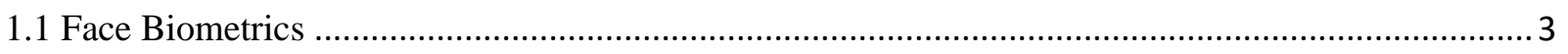

1.1.1 Advantages of Face Recognition Systems …............................................................... 4

1.1.2 Applications of Face Recognition Systems............................................................... 5

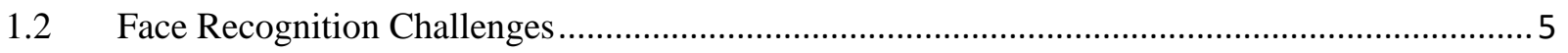

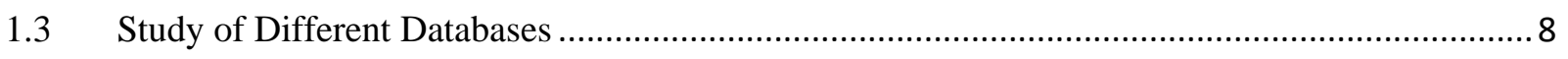

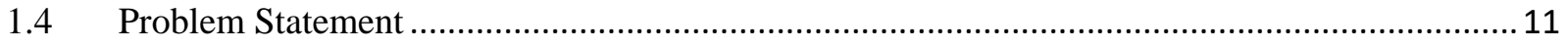

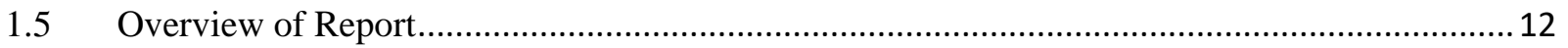

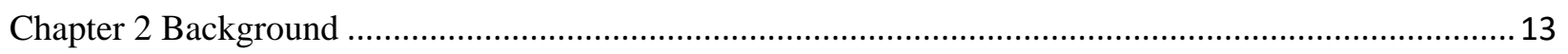

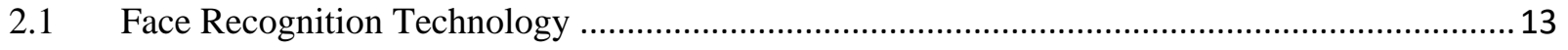

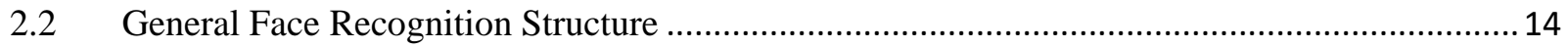

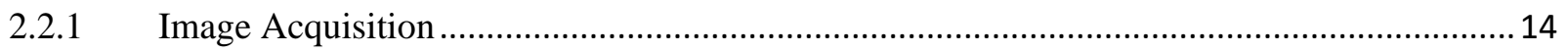

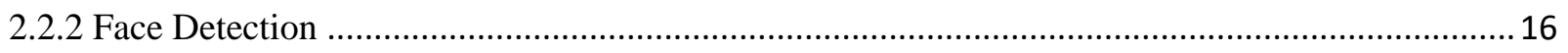

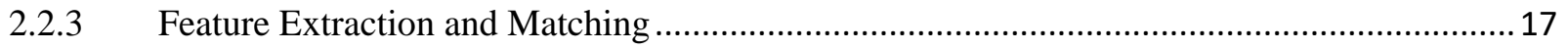

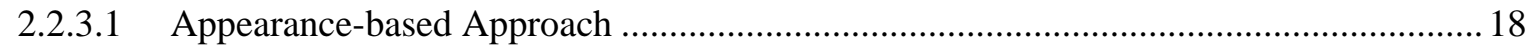

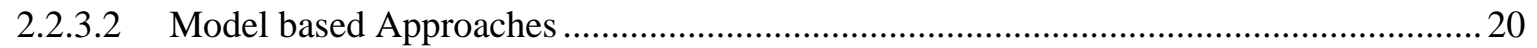

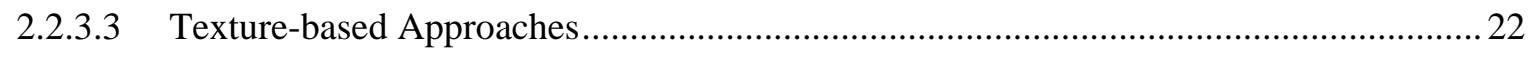

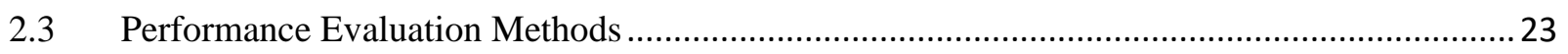

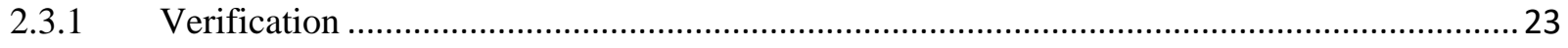

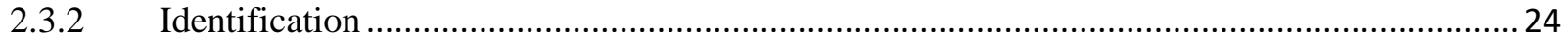

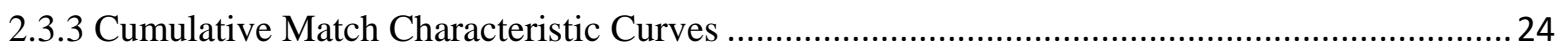

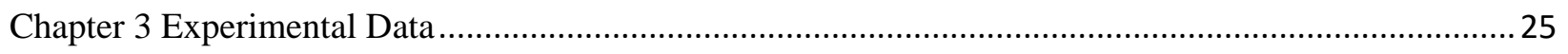

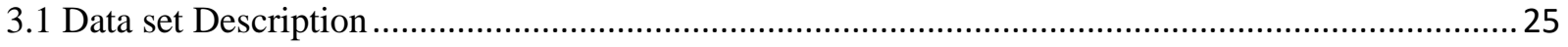

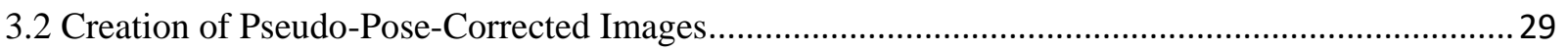

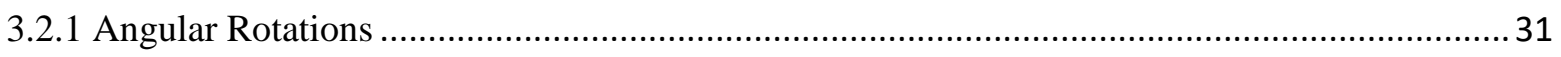

3.3 Creation of Pose-Corrected Images Using Cognitec DB Scan ................................................. 36 


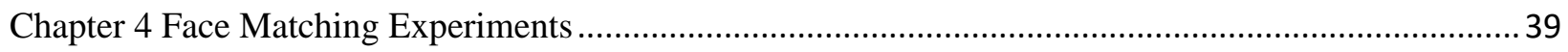

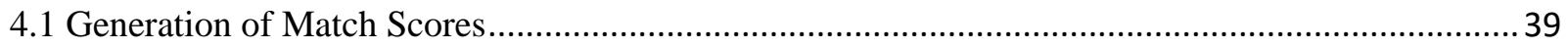

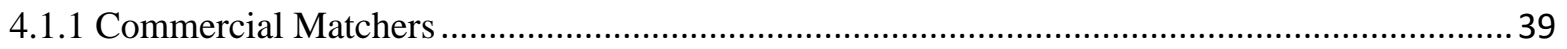

4.1.1.1 MegaMatcher SDK Matching Software..................................................................... 39

4.1.1.2 Cognitec FaceVACs SDK Matching Software …........................................................ 42

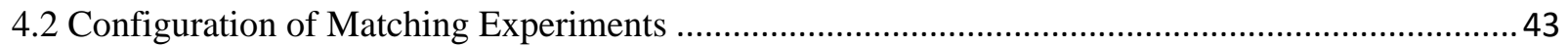

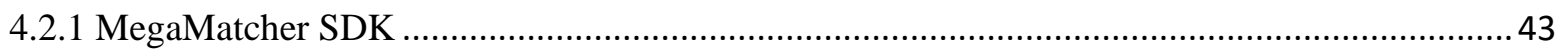

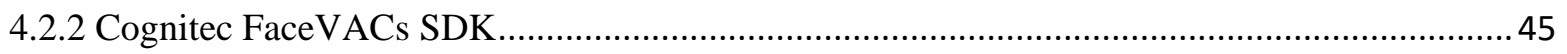

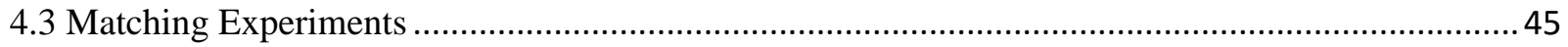

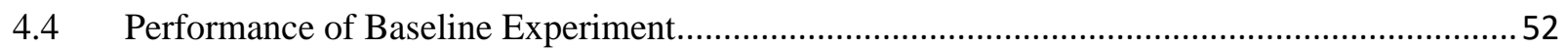

4.5 Performance Evaluation for Pose Varying Images ..........................................................52

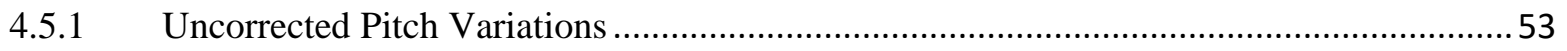

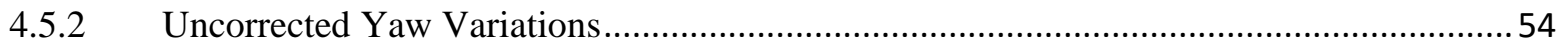

4.5.3 Uncorrected Coupled (Pitch and Yaw) Variations...........................................................56

4.6 Performance Evaluation for Pseudo Pose Corrected Images ................................................. 57

4.6.1 Performance of Pseudo Pose Corrected Pitch Variations Images .....................................5 57

4.6.2 Performance of Pseudo Pose Corrected Yaw Variations Images .................................... 61

4.6.3 Performance of Pseudo Pose Corrected Pitch and Yaw Variations Images.......................64

4.7 Performance of Pose Corrected Images Using Cognitec DB Scan Pose Normalization ............68

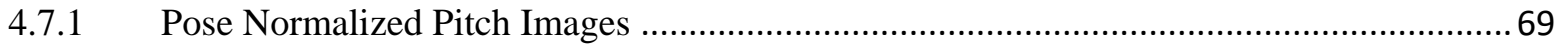

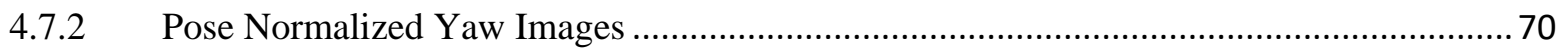

4.7.3 Pose Normalized Coupled Pitch and Yaw Images....................................................... 71

Chapter 5 Conclusions and Future Work ……............................................................................... 74

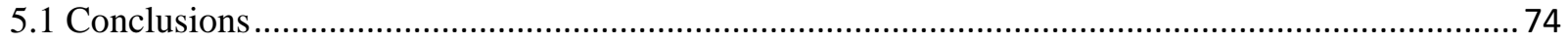

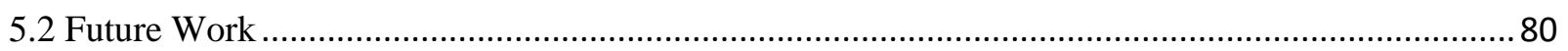

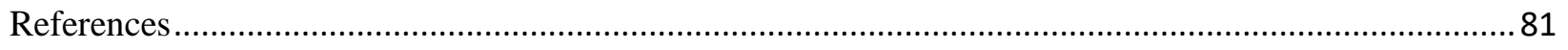




\section{List of Figures}

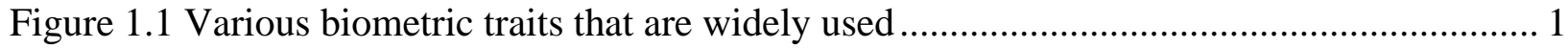

Figure 2.1 General Face Recognition Structure.................................................................. 14

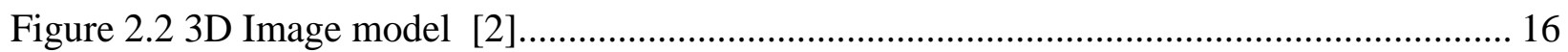

Figure 2.3 The Viola-Jones method of detection using windows of different sizes [2] ............. 17

Figure 2.4 Classification of Feature Extraction Methods ....................................................... 18

Figure 2.5 Eigen Faces................................................................................................... 19

Figure 2.6 Elastic Bunch Graph Matching of a Face.......................................................... 21

Figure 2.7 Illustration of LBP with an example ............................................................ 23

Figure 3.1 Canon EOS 5D Mark III camera and Canon EF 70-200mm f/2.8L IS III USM

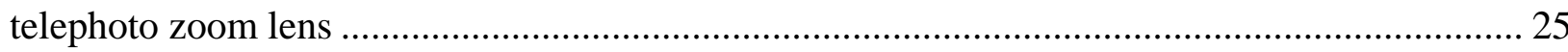

Figure 3.2 3dMD Face System [54] .............................................................................. 26

Figure 3.3 Pie chart Representation of Gender, Age, Ethnicity of the Total Data Collected. (a)

Gender, (b) Age, (c) Ethnicity ......................................................................................... 27

Figure 3.4 Pie chart Representation of Gender, Age, Ethnicity of the Selected Data in the Project.

(a) Gender, (b) Age, (c) Ethnicity .............................................................................. 28

Figure 3.5 Illustration of steps in generation of pseudo pose corrected image from 3D image (a)

Reading 3d files into MeshLab (b), (c) Applying Transform rotate filter (d) Marking the occlusion regions (e), (f) Applying 'Quadric Edge Collapse Decimation' ............................... 31

Figure 3.6 Orientation of head in terms of Pitch, Roll and Yaw angular rotations [56].............. 31

Figure 3.7 Pitch Varying Pose and Pseudo Pose Corrected Images .......................................... 32

Figure 3.8 Yaw Varying Pose and Pseudo Pose Corrected Images.......................................... 33

Figure 3.9 Pitch and Yaw Varying Pose and Pseudo Pose Corrected Images............................ 34

Figure 3.10 Steps in Generation of Pose Normalized Images from DB Scanner (a) Steps to read a probe image into Cognitec DB Examiner, (b) Applying the pose normalization filter in DB

Scanner, (c) Saving the generated normalized image .................................................... 37

Figure 4.1 Schematic representation of NeuroTechnology Biometric SDK [57]..................... 40

Figure 4.2 Flow Diagram of VeriLook Matching Mechanism [57] ....................................... 41

Figure 4.3 FaceVACs architecture: Feature set creation [58] .......................................... 42

Figure 4.4 FaceVACs architecture: Enrollment and Identification [58].................................. 43

Figure 4.5 Pose Varying Pitch angles ................................................................................ 48

Figure 4.6 Pose Varying Yaw Angles............................................................................... 50

Figure 4.7 Pose Varying Coupled Pitch and Yaw Angles ...................................................... 51

Figure 4.8 CMC Curve representation for baseline experiments, (a) CMC Curve for baseline experiment using Cognitec FaceVACs, (b) CMC Curve for baseline experiment using

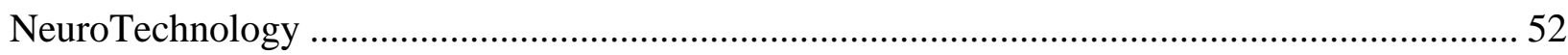


Figure 4.9 CMC curve representation of Uncorrected Pitch Variations Using Cognitec and NeuroTechnology Matchers, (a) CMC for Uncorrected Pitch Variations Using Cognitec FaceVACs, (b) CMC for Uncorrected Pitch Variations Using NeuroTechnology .... 53 Figure 4.10 Match Score Distribution of Uncorrected Pitch Variations, (a) Variation in match score for pitch angles from $10^{\circ}$ to $60^{\circ}$ using Cognitec FaceVACs, (b) Variation in match score for pitch angles from $-10^{\circ}$ to $-60^{\circ}$ using Cognitec FaceVACs .... 54 Figure 4.11 CMC curve representation of Uncorrected Yaw Variations Using Cognitec and NeuroTechnology Matchers, (a) CMC for Uncorrected Yaw Variations Using Cognitec FaceVACs, (b) CMC for Uncorrected Yaw Variations Using NeuroTechnology .... 55 Figure 4.12 Match Score Distribution of Uncorrected Yaw Variation Using Cognitec FaceVACs 55

Figure 4.13 CMC curve representation of Uncorrected Pitch and Yaw Variations Using Cognitec and NeuroTechnology Matchers, (a) CMC for Uncorrected Pitch and Yaw Variations Using Cognitec FaceVACs, (b) CMC for Uncorrected Pitch and Yaw Variations Using NeuroTechnology 56

Figure 4.14 Match Score Distribution of Uncorrected Coupled Pitch and Yaw Variations Using Cognitec FaceVACs, (a) Match Score Variation of Enrolled Positive Coupled Pitch and Yaw variations, (b) Match Score Variation of Enrolled Negative Coupled Pitch and Yaw variations 57 Figure 4.15 Pseudo Pose Corrected Pitch at Varying Polygon Reduction 58 Figure 4.16 CMC of Pseudo Pose Corrected Pitch at 25\% Polygon Reduction Using Cognitec and NeuroTechnology Matchers, (a) CMC of Pseudo Pose Corrected Pitch at 25\% Polygon Reduction Using Cognitec FaceVACs, (b) CMC of Pseudo Pose Corrected Pitch at 25\% Polygon Reduction Using NeuroTechnology 58 Figure 4.17 CMC of Pseudo Pose Corrected Pitch at 50\% Polygon Reduction Using Cognitec and NeuroTechnology Matchers, (a) CMC of Pseudo Pose Corrected Pitch at $50 \%$ Polygon Reduction Using Cognitec FaceVACs, (b) CMC of Pseudo Pose Corrected Pitch at 50\% Polygon Reduction Using NeuroTechnology 59 Figure 4.18 CMC of Pseudo Pose Corrected Pitch at 75\% Polygon Reduction Using Cognitec and NeuroTechnology Matchers, (a) CMC of Pseudo Pose Corrected Pitch at $75 \%$ Polygon Reduction Using Cognitec FaceVACs, (b) CMC of Pseudo Pose Corrected Pitch at $75 \%$ Polygon Reduction Using NeuroTechnology 60

Figure 4.19 Match Score Distribution of Pseudo Pose Corrected Pitch with Varying Polygon Count, (a) Match Score Variations in Pseudo Pose Corrected Pitch Using Cognitec FaceVACs, (b) Match Score Variations in Pseudo Pose Corrected Pitch Using NeuroTechnology ............... 60 Figure 4.20 Pseudo Pose Corrected Yaw at Varying Polygon Reduction 61 Figure 4.21 CMC of Pseudo Pose Corrected Yaw at 25\% Polygon Reduction Using Cognitec and NeuroTechnology Matchers, (a) CMC of Pseudo Pose Corrected Yaw at 25\% Polygon 
Reduction Using Cognitec FaceVACs, (b) CMC of Pseudo Pose Corrected Yaw at 25\% Polygon Reduction Using NeuroTechnology 62

Figure 4.22 CMC of Pseudo Pose Corrected Yaw at 50\% Polygon Reduction Using Cognitec and NeuroTechnology Matchers, (a) CMC of Pseudo Pose Corrected Yaw at 50\% Polygon

Reduction Using Cognitec FaceVACs, (b) CMC of Pseudo Pose Corrected Yaw at 50\% Polygon Reduction Using NeuroTechnology 63

Figure 4.23 CMC of Pseudo Pose Corrected Yaw at 75\% Polygon Reduction Using Cognitec and NeuroTechnology Matchers, (a) CMC of Pseudo Pose Corrected Yaw at 75\% Polygon Reduction Using Cognitec FaceVACs, (b) CMC of Pseudo Pose Corrected Yaw at 75\% Polygon Reduction Using NeuroTechnology 63

Figure 4.24 Match Score Distribution of Pseudo Pose Corrected Yaw with Varying Polygon Count, (a) Match Score Variations in Pseudo Pose Corrected Yaw Using Cognitec FaceVACs, (b) Match Score Variations in Pseudo Pose Corrected Yaw Using NeuroTechnology 64

Figure 4.25 Pseudo Pose Corrected Pitch and Yaw at Varying Polygon Reduction.... 65 Figure 4.26 CMC of Pseudo Pose Corrected Pitch and Yaw at 25\% Polygon Reduction Using Cognitec and NeuroTechnology Matchers, (a) CMC of Pseudo Pose Corrected Pitch and Yaw at 25\% Polygon Reduction Using Cognitec FaceVACs, (b) CMC of Pseudo Pose Corrected Pitch and Yaw at 25\% Polygon Reduction Using NeuroTechnology

Figure 4.27 CMC of Pseudo Pose Corrected Pitch and Yaw at 50\% Polygon Reduction Using Cognitec and NeuroTechnology Matchers, (a) CMC of Pseudo Pose Corrected Pitch and Yaw at $50 \%$ Polygon Reduction Using Cognitec FaceVACs, (b) CMC of Pseudo Pose Corrected Pitch and Yaw at 50\% Polygon Reduction Using NeuroTechnology.....

Figure 4.28 CMC of Pseudo Pose Corrected Pitch and Yaw at 75\% Polygon Reduction Using Cognitec and NeuroTechnology Matchers, (a) CMC of Pseudo Pose Corrected Pitch and Yaw at 75\% Polygon Reduction Using Cognitec FaceVACs, (b) CMC of Pseudo Pose Corrected Pitch and Yaw at $75 \%$ Polygon Reduction Using NeuroTechnology

Figure 4.29 Match Score Distribution of Pseudo Pose Corrected Pitch and Yaw with Varying Polygon Count, (a) Match Score Variations in Pseudo Pose Corrected Pitch and Yaw Using Cognitec FaceVACs, (b) Match Score Variations in Pseudo Pose Corrected Pitch and Yaw Using NeuroTechnology 68

Figure 4.30 Pose Normalized Pitch Images Using Cognitec DB Scanner 69 Figure 4.31 CMC of Pose Normalized Pitch Using Cognitec and NeuroTechnology Matchers, (a) CMC of Pose Normalized Pitch Using Cognitec FaceVACs, (b) CMC of Pose Normalized Pitch Using NeuroTechnology .................................................................................................... 70 Figure 4.32 Pose Normalized Yaw Images Using Cognitec DB Scanner .................................. 70 Figure 4.33 CMC of Pose Normalized Yaw Using Cognitec and NeuroTechnology Matchers, (a) CMC of Pose Normalized Yaw Using Cognitec FaceVACs, (b) CMC of Pose Normalized Yaw Using NeuroTechnology 71 
Figure 4.34 Pose Normalized Pitch and Yaw Images Using Cognitec DB Scanner ....

Figure 4.35 CMC of Pose Normalized Pitch and Yaw Using Cognitec and NeuroTechnology Matchers, (a) CMC of Pose Normalized Pitch and Yaw Using Cognitec FaceVACs, (b) CMC of Pose Normalized Pitch and Yaw Using NeuroTechnology ................................................. 72 


\section{List of Tables}

Table 1.1 Illustration of Various Face Databases .................................................................. 9

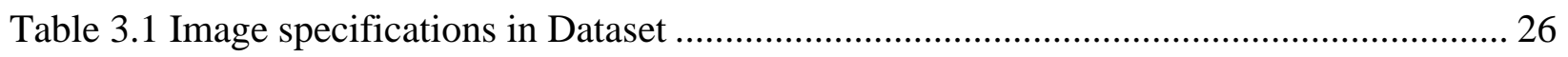

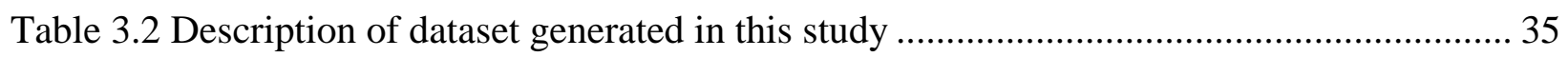

Table 4.1 Attributes of NeuroTechnology SDK used in this work [57] .................................... 44

Table 4.2 Attributes of Cognitec FaceVACs used in this work [58] ...................................... 45

Table 4.3 Number of images in each probe set for pitch rotation.......................................... 48

Table 4.4 Number of images in each probe set for yaw rotation .......................................... 50

Table 4.5 Number of images in each probe set for coupled (pitch and yaw) rotation................ 51

Table 5.1 Rank Accuracies of Various Pitch angle for Pseudo-Pose Corrected and Pose-Varying for Cognitec FaceVACs ..................................................................................................... 76

Table 5.2 Rank Accuracies of Various Pitch angle for Pseudo-Pose Corrected and Pose-Varying for NeuroTechnology ............................................................................................... 76

Table 5.3 Rank Accuracies of Various Yaw angle for Pseudo-Pose Corrected and Pose-Varying for Cognitec FaceVACs ................................................................................................ 77

Table 5.4 Rank Accuracies of Various Yaw angle for Pseudo-Pose Corrected and Pose-Varying for NeuroTechnology ............................................................................................... 78

Table 5.5 Rank Accuracies of Various Coupled Pitch/Yaw angle for Pseudo-Pose Corrected and Pose-Varying for Cognitec FaceVACs ................................................................................. 79

Table 5.6 Rank Accuracies of Various Coupled Pitch/Yaw angle for Pseudo-Pose Corrected and Pose-Varying for NeuroTechnology ..................................................................................... 79 


\section{Chapter 1 Introduction}

A biometric trait is a unique physical characteristic that can be used to identify an individual in an automated or semi-automated fashion. There are different biometric modalities that define the characteristics of an individual thereby defining the identity of that individual. Biometric attributes are often classified as physiological and behavioral [1]. Physiological attributes include face, fingerprints, iris patterns, and voice characteristics. Behavioral attributes include keystroke, and mouse dynamics, gait analysis and signature. Most widely used biometrics are fingerprints, voice, iris and face [2] (as shown in Figure.1.1).

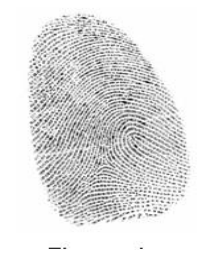

Fingerprint

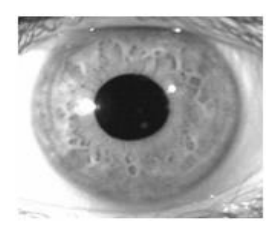

Iris

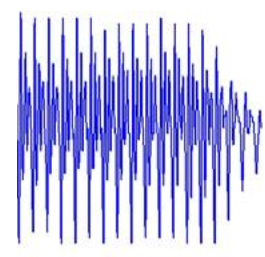

Voice

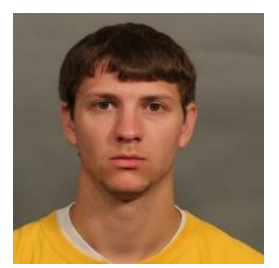

Face

Figure 1.1 Various biometric traits that are widely used

The performance of a biometric for any given application depends on the characteristics of the biometric and requirements of the application. A brief description of the most commonly used modalities (as seen the Figure 1.1) is given below:

Fingerprint: Fingerprint identification is one of the most familiar and commonly used biometrics. Because of their uniqueness and consistency, fingerprints have been used for identification for over a century [3]. Since the late 90's, fingerprint biometric technology is being automated enabled by advancements in computing capabilities. Other factors that have led to an increased use of 
fingerprints are the ease in acquisition, the numerous sources of data available for study and its established use.

Voice: Voice recognition is one of the biometric technology that uses an individual's voice for recognition. It can be classified as speech recognition and speaker recognition. Speech recognition is to detect a speech signal in the data which is either encrypted or recorded with noise. Speaker recognition is to identify an individual based on the voice in the data. It is a combination of both physical and behavioral biometric characteristics [4], where the physical characteristics are inherent to an individual based on the physical morphology of the vocal tract, but the behavioral characteristics of speech changes with age, medical conditions, emotional state, etc. The main issue in voice based recognition is the variation in compression and file types caused by different sources such as different cell phones or carriers and speaker emotion.

Iris: The iris is the circular region of the human eye located between the sclera and pupil of the eye. The iris controls the size of the pupil depending on the level of illumination coming into the eye. The intricate tissue structure of the iris is highly unique among the human population. Iris patterns are effective at differentiating between individuals as the iris pattern differs even for twins. The main challenges of iris recognition include poor illumination, motion blur, occlusion due to eyelashes, use of contact lens, etc. [5].

Face: Face is yet another common biometric that is used to identify people in everyday life. In spite of considerable improvement in recognition performance in recent years, facial recognition still remains challenging in many real-time operational applications. As the face information of an individual can be acquired without ones' knowledge, from a CCTV (Controlled Circuit Television) footage, video, camera, etc., there are many non-idealities that affect the performance of the face recognition [6]. The key concerns of face recognition are varying lightning, different backgrounds, varying pose angles, etc.

The goal of this problem report is to examine the performance of pose varying images using two commercial matchers Cognitec FaceVACs SDK (Software Development Kit), Cognitec FaceVACs DB Scanner and NeuroTechnology MegaMatcher. 
Initially, the pose varying 2D images are generated from 3D models and are pseudo pose corrected by varying the polygon count at the interpolated regions using Mesh Lab. Identification tests are run for these images against a gallery of 1203 2D Mugshot images in both Cognitec and NeuroTechnology matcher and the performance of these tests is evaluated using CMC curves and box plots. Cognitec FaceVACs DB Scanner comes with an inbuilt pose correction algorithm which is used to normalize varying pose images. These pose corrected images are also tested against the gallery of Mugshots in both the matchers and the performance is evaluated using CMC curves.

\subsection{Face Biometrics}

Face is the most common biometric employed for identifying a person. In our normal day-to-day life, we see and recognize many people based on their facial structure. Face as a biometric modality has a lot of applications in identifying an individual from a set of available entries in a database. As discussed earlier, there has been considerable advancement in the field of face biometrics over the past few decades. It is now a modality of biometrics that is automatically implemented in several applications worldwide. Also, advancements in the field of face recognition has made it a more secure method to safe guard information.

The concept of face recognition started in early 1960's when Bledsoe, along with Helen Chan and Charles Bisson started working on a computer to recognize faces [7], but, much of their work did not get published due to limitations imposed by the funding agency. Early face recognition techniques required manual location of features, key landmarks that are used to define a face. In 1970's Goldstein, Harmon and Lesk proposed a method that used a subjective description of facial features, such as long ears, wide-set eyes etc., which were to be manually extracted. Initially, they started using 22 features for a population of $4 \times 10^{6}$ individuals and predicted that only 14 features are required [8] to isolate a face from a population using feature descriptions. In 1973 Kanade proposed the first face recognition algorithm in which the features were automatically extracted [9]. The recognition performance was about $45 \%$ to $75 \%$, thus proving that by rejecting the irrelevant features it is possible to amplify the recognition performance. 
Automated face recognition has gained much attention and is being employed in experimentation in different ways. In 1987, Kirby and Sirovich [10] proposed the principal component analysis (PCA) based method, which has been the benchmark of face recognition for the next generation techniques of facial biometrics. From then on-, different approaches have been proposed which have brought revolutionary changes in the field of face recognition which include Eigen faces [11], LDA [12] (Linear discriminant analysis), LBP [13] (Linear binary patterns) and SVM [14] (Support vector machine) etc.

\subsubsection{Advantages of Face Recognition Systems}

Biometrics has multiple modalities, and each modality has a different set of properties which benefits a specific application as per the requirements. The use of face as a biometric has unique characteristics which provides advantages compared to other modalities which are discussed below.

- Face is the most common mode to recognize a person. In general, we remember a person by remembering his face. In biometrics, the machine learning algorithms are trained to recognize a person based on his/her features.

- The face modality is non-intrusive. This is one of the main reasons face is being chosen for many applications. The data acquisition for face recognition can be done from a distance without the knowledge of the subject and through a non-contact process. However, this can be a disadvantage at times as it affects the performance.

- Face databases are one of the most important property of face modality. In comparison to other biometric modalities face has many open sources of databases available. Face data acquisition and storage requires lot of efforts and is most important section in implementing any face recognition systems. With the availability of large datasets proves to be highly advantageous and aids in fast implementing of many applications.

- Face recognition systems provides fast and reliable results. In the recent times, many commercial firms are integrating a face recognition model into their security applications to obtain better results. 


\subsubsection{Applications of Face Recognition Systems}

Face recognition is a growing research area in the present world where many new applications emerge every day for different needs. Across the world variations in applications of face recognition techniques are shown as follows [15].

- Face recognition systems are frequently observed being used for surveillance applications. Varying lighting conditions, face orientations and factors make the distribution of face recognition system for large scale surveillance challenging. A case in Newham Borough of London town center surveillance has 300 cameras linked to closed circuit TV (CCTV) controller room. The authority claims that there is about $34 \%$ drop in crime with the use of this technology.

- All over the world many airports, commercial complex and social gathering events have started implementing face recognition systems as part of their protection systems. In October 2001 Fresno Yosemite International (FYI) airport in California has implemented Viisage's face recognition technology for airport security purposes which informs the airport public safety officers if features of an individual matches with a known terrorist at airport checkpoint.

- Access control is another common application of face recognition systems. In access control systems group of individuals who are to be recognized are relatively small. Data in such a system is gathered under controlled conditions, uniform background and frontal faces.

- Image database investigation is another application of face recognition systems which includes searching image databases of licensed drivers, missing children, immigrants and police bookings.

\subsection{Face Recognition Challenges}

Face recognition is mostly required in cases where an individual's data cannot be gathered with his/her will. At many public places, we do not have access to an individual from a distance that we can collect the data from them. In such situations, we look for characteristics like face or gait of 
the individual. In general, at data collection centers we take pictures of a subject at controlled lightning conditions, fixed background and with desired pose angles. But when we want to take pictures of a subject outside the collection laboratory there are many conditions that affect the quality of the picture, such as varying lightning, different backgrounds and varying pose angles. Other challenges that affect the performance of a face recognition system include scale, age, occlusions, blur, spoofing and noise. As of today, we have systems that work for images taken under constrained conditions. Identifying the individual in an unconstrained scenario is a much more desirable outcome for the law enforcement firms, authentication purpose and intelligence agencies. Work is being done to generate a model that can work robustly against these challenges. The various face recognition challenges are discussed below.

\section{Illumination:}

The variations in intensity of light and its direction can affect the illumination in the images captured. Performance variations of images of same individual due to illumination is sometimes larger than the variation due to the facial features. Same individual with the same facial expression would appear different with varying lighting directions and intensity [16]. In such cases the system would recognize images with similar illumination than due to similar facial features. Wang, Li and Yang sheng [17] has proposed a method using self-quotient image (SQI) for better performance under varying illumination conditions.

\section{Scale:}

Scale is an important factor to be considered while working with unconstrained images. In surveillance scenarios, capturing an image from 5 feet distance has a better scale/resolution than an image captured at a 50 feet distance. Scale reduction would result in loss of information which in turn affects the performance of the system. The problem of scale variation can be handled using feature-based detection algorithms as stated in [18] by Yow and Cipolla. Boom and Beumer [19] also worked on the low resolution/scale images to improve the performance while using such images. 


\section{Age:}

Human faces undergo a lot of changes over time which include facial texture, facial hair, presence of glasses, shape, etc. As face recognition has various applications like passports, license and surveillance videos it is a major challenge for a face recognition system to generate better results with varying age of the subject [20]. Work done on face recognition across ages is relatively less because of the lack of data sets. Narayanan Ramanathan and Rama Chellappa [21] have proposed a Bayesian age classifier, that classifies the face images of the subjects as per the difference in age and performs verification test across age progression.

\section{Occlusions:}

Face recognition collects the features in the human face to generate the test of recognizing the individual. Due to occlusions, desired features of facial data cannot be acquired as, people use accessories such as sunglasses, scarfs, and hats which partially obstruct the face region. In the process of data acquisition, anything that obstructs the subjects face in the projection of camera would result in an occlusion and this could be as simple as the hand movement of the subject. This results in loss of facial information at that location. Ekenel and Stiefelhagen [22] have implemented a local appearance-based algorithm to study the performance of images with occlusions.

\section{Pose:}

Pose is one of the most prominent challenges in face recognition systems. The problem of pose occurs due to the 3D construction of human faces. Pose variations refers to the rotation of facial image from the planar angle. Greater the angle of rotation greater is the drop-in performance of face recognition systems. Variations in pose results in change in the appearance of human face and even the most sophisticated systems fail in detecting this without a proper method of pose correction technique.

Lot of study has been done and is still going on in finding a proper solution for images with arbitrary poses. Feature extraction techniques can be implemented to generate a frontal pose image 
from pose varying images [23]. 3D face recognition techniques are gaining mare attention these days within the area of pose varying face recognition. Construction of 3D Morphable models to aid in the recognition of image with arbitrary pose was implemented by Blanz and Vetter in [24], where they have presented the results of identification test over 4,488 images from CMU-PI dataset and 1,940 images from FERET dataset which are publicly available.

\section{Blur:}

Working with unconstrained images taken at random conditions would result in image degradation due to blur. Images acquired with blur results in loss of information of facial features. Blur mainly occurs because of unwanted motion of the subject or due to the atmospheric conditions where data is collected from surveillance source or a motion camera. To achieve better performance with the images having blur, Heflin et al [25] proposed methods for estimating motion blur and atmospheric blur which are fully automated and easily merge with a real-time face recognition system.

\section{Face Image Spoofing:}

Unlike other biometric modalities, face image can be easily obtained and so it is more susceptible to spoofing attacks. With the growth of social media and use of advance mobile devices, it has become easy to obtain the images of a person making way for spoofing attacks. However, there has been attempts to understand and solve the problem of spoofing where Di Wen, Anil K. Jain et al [26] have proposed a robust face spoof detection algorithm based on image distortion analysis (IDA). They stated that the proposed method on two publicly available databases has outperformed the existing spoof detection methods.

\subsection{Study of Different Databases}

As discussed earlier one of the advantages of considering face recognition study is the availability of several pre-acquired databases. Some of the most used databases and their characteristics are discussed as follows in Table 1.1 [27] 
Table 1.1 Illustration of Various Face Databases

\begin{tabular}{|c|c|c|}
\hline Dat & Description & Characteristics \\
\hline $\begin{array}{c}\text { AT\&T Database } \\
\text { (ORL Database) } \\
{[28]}\end{array}$ & $\begin{array}{l}\text { AT\&T database of faces contains a set of } \\
\text { face images taken from April } 1992 \text { and April } \\
1994 \text { with } 10 \text { different images for each of } 40 \\
\text { distinct images with a homogenous black } \\
\text { background. Of this some of the subjects' } \\
\text { images are collected at different times, } \\
\text { varying lighting, facial expression and facial } \\
\text { details (glasses/no glasses) with fixed } \\
\text { upright, frontal position. }\end{array}$ & $\begin{array}{l}\text { This database contains } \\
\text { images with varying } \\
\text { illumination and } \\
\text { expression which aids in } \\
\text { the study of } \\
\text { unconstrained images } \\
\text { with these characteristics. } \\
\text { This database does not } \\
\text { possess any data with } \\
\text { varying poses. }\end{array}$ \\
\hline $\begin{array}{r}\text { FERET Da } \\
\text { [29] }\end{array}$ & $\begin{array}{l}\text { The database is created by the FERET } \\
\text { program from } 1993 \text { to } 1997 \text { having about } \\
1000 \text { subjects to aid government monitored } \\
\text { testing and evaluation of face recognition } \\
\text { algorithms. The final set of database consists } \\
\text { of around } 14051 \text { grayscale images of human } \\
\text { face with views that include frontal views, } \\
\text { right and left profiles views and quarter left } \\
\text { and right views. It also has images of same } \\
\text { subjects which are obtained with a time } \\
\text { interval of one or more years to observe the } \\
\text { change in facial features with age } \\
\text { progression. }\end{array}$ & $\begin{array}{l}\text { This data set does not } \\
\text { have wide range of pose } \\
\text { varying images and has } \\
\text { no clear details about the } \\
\text { illumination of the } \\
\text { images in the database. }\end{array}$ \\
\hline $\begin{array}{l}\text { CMU Pose, } \\
\text { Illumination and }\end{array}$ & $\begin{array}{l}\text { PIE database is collected in } 2000 \text { with } 68 \\
\text { subjects. This collection has taken place at } \\
\text { CMU with facial images of each person }\end{array}$ & $\begin{array}{l}\text { This dataset does not } \\
\text { discuss about background }\end{array}$ \\
\hline
\end{tabular}




\begin{tabular}{|c|c|c|}
\hline $\begin{array}{c}\text { Expression (PIE) } \\
\text { Database [30] }\end{array}$ & $\begin{array}{l}\text { having } 13 \text { different poses, } 43 \text { different } \\
\text { illuminations and } 4 \text { different facial } \\
\text { expressions with over } 40,000 \text { facial images. } \\
\text { In this database two sets of images were } \\
\text { captured, one with ambient light and the } \\
\text { other in the absence of ambient light. }\end{array}$ & $\begin{array}{l}\text { conditions and the } \\
\text { unspecified pose angles. }\end{array}$ \\
\hline $\begin{array}{c}\text { Yale Face Database } \\
\qquad[31]\end{array}$ & $\begin{array}{l}\text { Yale Database has a total of } 165 \text { facial } \\
\text { grayscale images of } 15 \text { individuals in GIF } \\
\text { format. Each subject in this data set has } 11 \\
\text { images, each image with different facial } \\
\text { expression (happy, sad, sleepy, surprise and } \\
\text { wink), facial detail (w, w/o glasses) and } \\
\text { varying lighting conditions (center, left and } \\
\text { right). }\end{array}$ & $\begin{array}{l}\text { Very small number of } \\
\text { subjects and has no } \\
\text { information about pose } \\
\text { variations. }\end{array}$ \\
\hline $\begin{array}{c}\text { Yale Face Database } \\
\text { B [32] }\end{array}$ & $\begin{array}{l}\text { The database contains images of } 10 \text { subjects } \\
\text { with } 64 \text { different lighting angles and } 9 \\
\text { different pose angles. For every subject in } \\
\text { particular pose an image with ambient light } \\
\text { was also generated making a total of } 5850 \\
\text { images. In the } 9 \text { different poses } 0 \text { degrees is } \\
\text { the frontal view and for poses } 1 \text { to } 5 \text { the } \\
\text { subject views at } 5 \text { points on a semicircle with } \\
12^{\circ} \text { from the camera lens in the left visual } \\
\text { field and for the rest the subject views at } 3 \\
\text { points on a semicircle at } 24^{\circ} \text { from the camera } \\
\text { lens, again in the left visual field. To capture } \\
\text { the images an overhead lighting structure } \\
\text { fitted with } 24 \text { computer controlled xenon }\end{array}$ & $\begin{array}{l}\text { Very small number of } \\
\text { subjects. Pose angles in } \\
\text { this data are not precisely } \\
\text { controlled. }\end{array}$ \\
\hline
\end{tabular}




\begin{tabular}{|c|c|c|}
\hline & $\begin{array}{l}\text { strobe lights was used at a rate of } 30 \\
\text { frames } / \mathrm{sec} \text {. }\end{array}$ & \\
\hline $\begin{array}{c}\text { XM2VTS Database } \\
\text { [33] }\end{array}$ & $\begin{array}{l}\text { This database provides synchronized video } \\
\text { and speech data along with image sequences } \\
\text { allowing multiple views of faces. It consists } \\
\text { of digital video records of } 295 \text { subjects } \\
\text { collected over a period of } 5 \text { months with an } \\
\text { interval of one month. Significant changes in } \\
\text { facial hair, hairstyle and accessories can be } \\
\text { observed in this data. }\end{array}$ & $\begin{array}{l}\text { Details of pose variations } \\
\text { are not discussed; no } \\
\text { information is provided } \\
\text { regarding the illumination } \\
\text { and background. }\end{array}$ \\
\hline
\end{tabular}

\subsection{Problem Statement}

As discussed earlier face as a biometric is one of the most emerging areas in biometrics. Ideal face recognition systems must be robust and should deliver optimal results for any mode of operation. But the present-day face recognition systems are influenced by various factors as seen earlier. Of all the factors the main concern is pose varying images. In general, when images are collected from a video or a camera footage, those 2D images occur at different facial poses and correcting such images is highly difficult. Trying to correct the varying pose images cannot produce the original image, because those 2D images with non-frontal views do not have the complete facial information [34]. In such cases adopting 3D models can help generate the frontal face. 3D models contain information of all the three dimensions and so they can produce frontal face from any pose.

Identifying a person with varying pose would help identifying people from crowds and many other situations. In real world scenarios, the available information to identify a person may not be a perfect image taken under desired conditions, there may be pose variations, occlusions, illumination variations and many other factors. Therefore, the uncontrolled nature of the nonidealities in the face data will impact the performance of the face recognition system. 
The goal of this work is to evaluate the effectiveness of commercial pose-correction software using $2 \mathrm{D}$ non-frontal pose images generated using 3D facial scans. The performance is evaluated based on the match scores generated from two matchers. Identification mode of face recognition is adopted for this process to generate the match scores. The evaluation of match scores is done using the cumulative match characteristic (CMC) curves.

\subsection{Overview of Report}

This chapter gives a brief overview of face biometrics, face recognition scenarios and challenges of face recognition systems. The main idea of this work is also discussed in this chapter. The remainder of this report is organized as follows,

\section{- Chapter 2}

This chapter gives a summary of the work done in the areas of face recognition on unconstrained facial images. Work related to 3D models, face recognition using 3D models and about pose correction of images using 3D models is discussed here.

\section{- Chapter 3}

Describes about the generation of a data set of pose varying $2 \mathrm{D}$ images generated from 3D data and method implemented to generate pseudo pose corrected images in the data set.

\section{- Chapter 4}

Describes the experimental procedure with the two commercial matchers NeuroTechnology MegaMatcher and the Cognitec FaceVACs Matcher and their performance. Summary of the experimental results generated from the commercial matcher using the biometric data.

\section{- Chapter 5}

This chapter gives the overview of the contributions of the work and discusses the best possible developments for this work in the future. 


\section{Chapter 2 Background}

\subsection{Face Recognition Technology}

Face recognition is a well-established field of research with many open challenges remaining. Face recognition is a subfield of pattern recognition and computer vision which uses statistical methods to perform detection and extraction of features from image data to match with the patterns stored in a database. Face recognition systems operating on high quality image data can identify an individual based on the pattern of features that comprise the face. As discussed in the introductory chapter, recognition performance degrades when non-idealities are present in the image, including: inadequate or non-uniform lightning conditions, low sensor resolution, and non-frontal pose angle.

Face recognition processes are performed in sequential order. The initial step is to acquire an image for testing (the 'probe' image), and this acquisition can be done using a camera or a video sequence. The probe image then undergoes face detection and feature extraction where the face is detected within the area of probe image which may contain backgrounds, other faces and even more complex patterns. After this, the face image is normalized to the extent possible to retain the information of the face required to recognize the individual There are different normalization techniques that are implemented such as histogram equalization to improve the image contrast, intensity normalization to equalize the intensity of images and many other types. Then the normalized image is passed on to the software which creates a template to compare against the available set of gallery image to perform a verification or identification match. 


\subsection{General Face Recognition Structure}

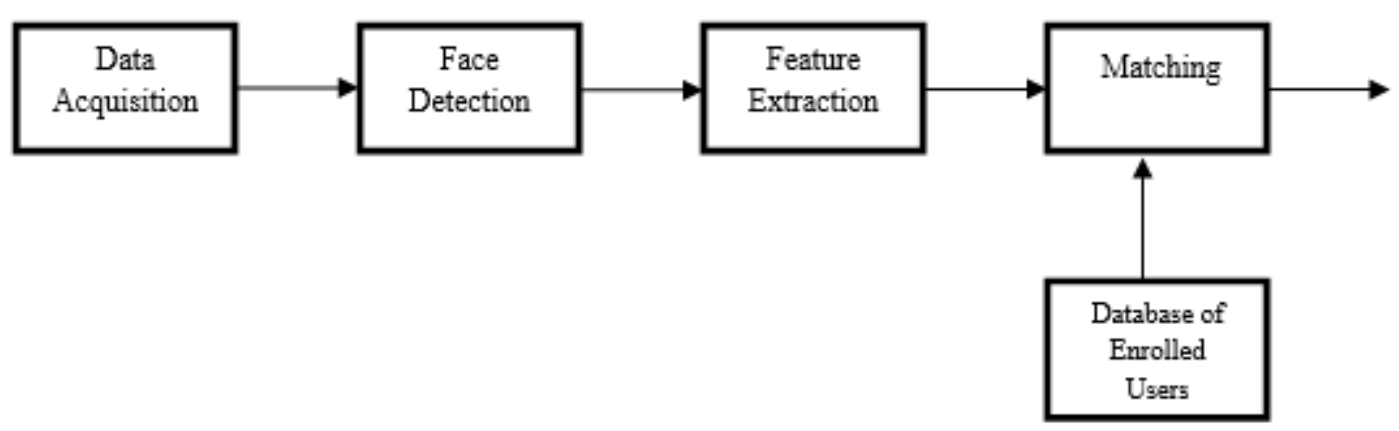

Figure 2.1 General Face Recognition Structure

A general face recognition system consists of 4 major steps which are: image acquisition, face detection, face alignment, and feature extraction. These steps are performed prior to the face recognition on the query image resulting in the final decision. In the following sections, these modules of the face recognition system are discussed in brief.

\subsubsection{Image Acquisition}

To perform a face recognition test, a set of images are required over which the test image is compared with the existing database, and image acquisition is performed using a still frame or video camera. The matching performance of a facial recognition system depends on the quality of the images used in the gallery and probe. The presence of non-uniform illumination, variations in background, non-frontal pose angles, and non-neutral facial expressions can decrease the match performance of the system. In general, collection of gallery and probe images under constrained conditions will give the best matching performance for any given application. Working with facial images collected in unconstrained conditions is more challenging for a system than the images which are acquired under controlled conditions

Image acquisition involves use of different sensors according to which the resulting data would be an ordinary $2 \mathrm{D}$ image or a $3 \mathrm{D}$ model or a sequence of images [2]. 


\section{D Image Sensors:}

2D images have been, and still are, a prominent source for the face recognition systems. The sensors designed for face recognition systems use visible spectrum 2D mugshot images for recognition. With two-dimensional images, all the data of a face image cannot be captured and there occurs loss of data due to occlusion and 3D facial geometry. In order to eliminate the effect of pose and illumination, multi cameras are used to capture the face images at different angles.

Video sequences can be defined as a part of 2D images. Surveillance cameras are often employed for continuous recording of human activity. In such scenarios, frames of video sequences containing facial images are used as probe images to ascertain the identity of an individual. In the input of the video, the system should also be able to track the faces apart from detecting the faces, which is essentially a motion evaluation problem. In [35] [36] Zhao \& Chellapa et al, have stated that face tracking can be done as feature tracking, head racking, and model-based tracking. In face tracking, initially the location of face is estimated by calculating the variations in frames. This process may be affected by changes in illumination, pose variation and computation of frame rate etc.

\section{D Image Sensors:}

Recent developments have shown that 2D images could be replaced by 3D models. The image captured using a 3D sensor cover about $120^{\circ}$ of human head and is referred as $2.5 \mathrm{D}$ scan and a full view of a face can be constructed by combining 2-3 2.5D scans from multiple views [2] [35]. In general, a 3D model is represented as a polygonal mesh (triangular or rectangular form) as shown in the Figure 2.2. The 3D mesh structure can be altered based on the preprocessing (e.g., smoothing, filling holes, etc.), mesh construction, and imaging process (scanning with laser sensor) [24]. 

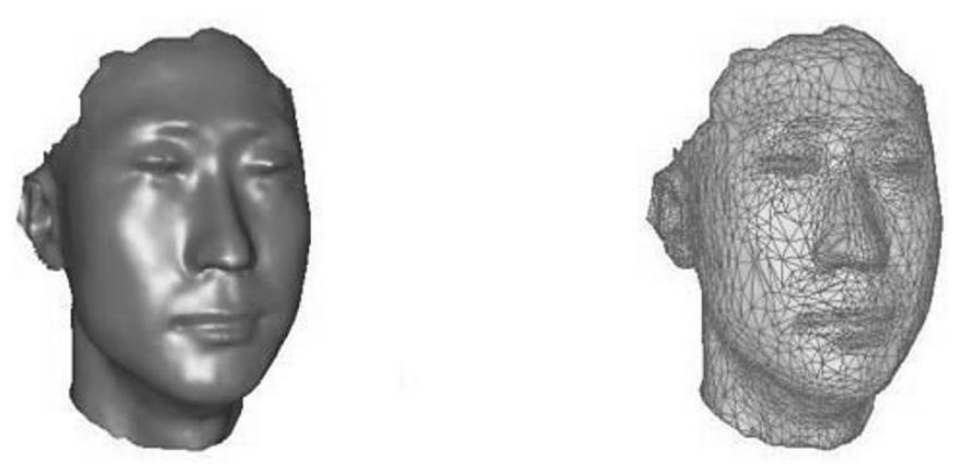

Figure 2.2 3D Image model [2]

\subsubsection{Face Detection}

Face detection is a software-implemented module in which it detects the facial features and ignores all other data in the image. Face detection is regarded as a specific case of object-class recognition, a significant task in computer vision. It is challenging for face detection software to extract the features of a face in conditions of varying pose with partial or complete occlusion of facial features, with cluttered background, and non-uniform illumination. Many approaches have been proposed for face detection and normalization [37]. In the present day, most detection algorithms use local texture-based features and a binary classifier to differentiate face and a non-face [2] [38] [39]. In the real-time object detection, the work done by Viola and Jones is widely used because of high accuracy and availability as an open source tool.

\section{Viola Jones Face Detector:}

Viola and Jones proposed the face detection algorithm in 2003 [40]. This method scans the input image with detection windows of different sizes to identify if there is a face in the image. In this process, the existence of a face is determined by applying a classifier of simple local features for each window using rectangular filters. The rectangular filters can be classified as two-rectangle, three-rectangle and four-rectangle. The process of scanning a facial image with detector windows using Viola Jones algorithm is shown in Figure 2.4. 


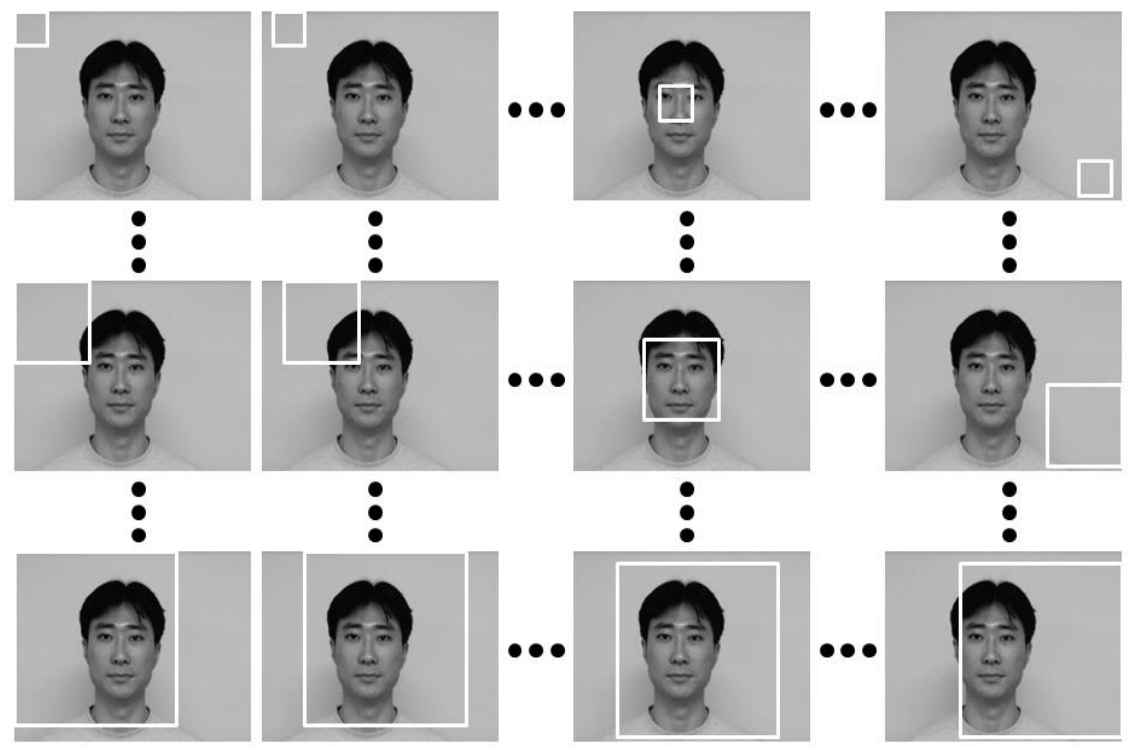

Figure 2.3 The Viola-Jones method of detection using windows of different sizes [2]

The rectangular filters applied in this method are two dimensional, and are similar to one dimensional Haar Wavelets which are used in signal processing domain and are also called as Haar-like filters [2] [41]. The Haar-like filters are applied to each window and the weighted sum is calculated such that if the sum is greater than a certain threshold then the window is considered to have a face image.

\subsubsection{Feature Extraction and Matching}

Features are essential in classifying and recognition of images. They define the desired pattern in an image, making it easy to work within an automated environment. In pattern recognition and image processing, feature extraction is considered a special form of dimensionality reduction. Converting the information in an image into a set of features is defined as feature extraction. Many algorithms are available for this feature extraction. State of the art feature extraction algorithms are updated versions of the ones developed years ago. Principal Component Analysis (PCA) is one such algorithm. It was invented a century ago, was used for pattern recognition in 1965 [42], and was applied to facial recognition in the early 90's [10] [11]. There are three main approaches, or 
classes, to extract features and match images. These feature extraction classes are illustrated in the image below, and discussed in the following sections.

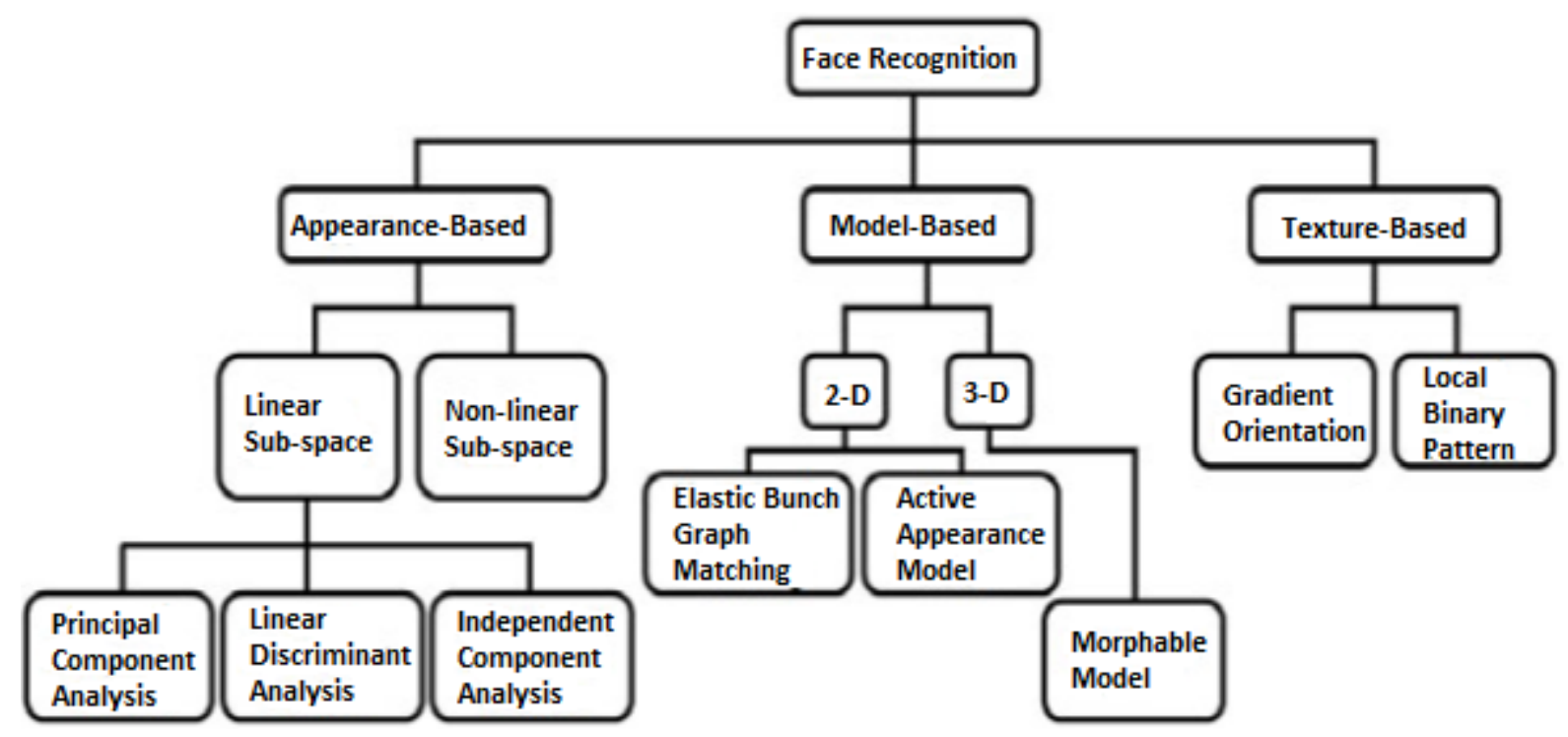

Figure 2.4 Classification of Feature Extraction Methods

\subsubsection{Appearance-based Approach}

This approach is based on two-dimensional images. Appearance-based methods can be categorized into linear and nonlinear types. The linear appearance-based method implements linear dimensional reduction. In comparison, the non-linear appearance-based method is highly complex. Commonly used mapping techniques are Principal Component Analysis (PCA), Linear Discriminant Analysis (LDA) and Independent Component Analysis (ICA). In an appearancebased approach, the whole face region from an image is used to generate a compact structure by mapping a high-dimensional image into a low-dimensional subspace [2]. A subspace is the representation of basic vectors which are derived using a training set of images. In this method, the face is represented as a set of raw intensity images and each image reflects a high-dimensional vector. Then, statistical techniques are applied to extract a feature space from the image distribution, and the sampling image is compared against the training data set. 


\section{Principal Component Analysis}

Principal Component analysis is a subspace projection technique [43] which is one of the most widely-used facial recognition techniques. It is generally referred to as the use of Eigenfaces, and is based on a mathematical procedure which performs dimensionality reduction of data using data compression basics, and extracts the principal components of multi-dimensional data.

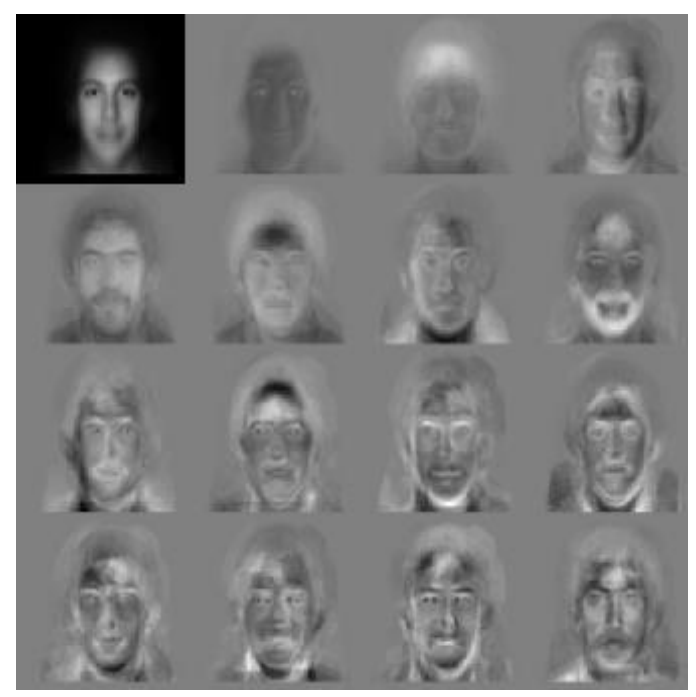

Figure 2.5 Eigen Faces

This approach involving reduction in dimensions gets rid of the information that is not useful and precisely decomposes the face structure into orthogonal/uncorrelated components known as Eigen faces. A probe image is compared against a gallery image by measuring the distance between their respective feature vectors [43]. The PCA approach generally requires the full-frontal face to be presented each time, which is one of its drawbacks. Another disadvantage is that this method is not resistant to variation in lighting and expressions. The primary advantage of this technique is that the data can be reduced up to 1/1000th of the original size.

\section{Linear Discriminant Analysis}

LDA involves a statistical approach for classifying samples of an unknown class based on samples of a known class. It aims at increasing between-class variance and reducing within-class variance [43]. 
This method is largely used when accelerated matching process is required. Standard LDA is designed considering only two classes, but multi-class projection vectors are used more commonly. Different projection vectors are generated for different classes. This technique needs larger databases, because when high-dimensional face data is present, the available sample size might be less than that compared to the dimensionality of the sample space. This method, like PCA, can deal only with frontal images. The advantage of LDA is that it is resistant to changes in lighting and expressions. Various experiments requiring small training sets proved that results obtained using PCA are better than those obtained due to LDA [44].

\section{Independent Component Analysis}

Independent component analysis (ICA) of a random vector includes searching for a linear transformation which minimizes the statistical dependence between its components [45]. This method can be seen as an extension of PCA, where PCA is operated only on models up to second order statistics whereas ICA can be operated on higher order statistics [46].

\subsubsection{Model based Approaches}

Recognition of facial expression is necessary for designing any human interface systems. The previously stated approach works only when the person faces the camera i.e., the approach works only with frontal images [47]. This method tries to model a face adequate to recognize an image. This method deals with all kinds of images and uses multiple landmark points on face such as tip of the nose, corners of eyes and mouth, etc. making it a more reliable matching technique than appearance-based approaches. The model-based approach is based on three-dimensional face models along with two-dimensional images [24]. Realistic animation of faces can also be generated with this approach [2]. Some examples of this approach include face bunch graphs (FBG) and active appearance model (AAM) [48].

\section{Elastic Bunch Graphs (EBGM)}

EBGM is a model-based approach that depends on the concept that real face images have many nonlinear characteristics which are not addressed by the linear analysis methods discussed earlier, 
such as variations in illumination, pose and expression. This method uses the convolution of an image along with a Gabor filter, which is used to detect shapes and to extract features using image processing. A Gabor jet is a node on the elastic grid which describes the image behavior around a given pixel [2] [49]. Recognition is based on the similarity of the Gabor filter response at each Gabor node. The difficulty with this method is the requirement of accurate landmark localization, which can sometimes be achieved by combining PCA and LDA methods [43].

The structural knowledge of faces and their variants are represented as a bunch graph. A face bunch graph can be modeled in two phases. In the first phase, the user manually marks the required landmarks and defines the geometric structure of the image graph of the first image. This graph helps the remaining images in the training set to semi-automatically develop their respective image graphs. The second phase includes acquiring the face bunch graph from a set of corresponding image graphs. [2] [4].

An edge between two nodes of the face bunch graph (FBG) is assigned based on the average distance between corresponding nodes in the training set [2]. Landmark points for a new image are obtained by increasing the similarity between the FBGs of alike poses [4]. This process is known as elastic bunch graph matching (EBGM). The precise marking of landmarks plays an important role in this matching process.

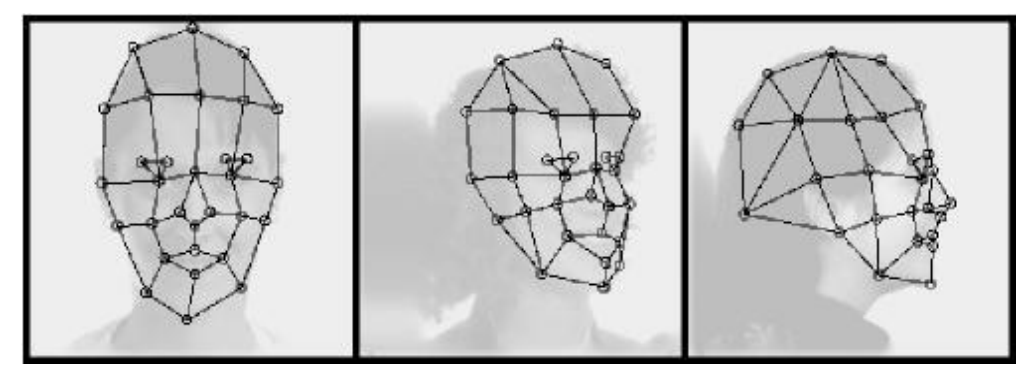

Figure 2.6 Elastic Bunch Graph Matching of a Face

\section{Morphable Model}

In most operational scenarios, the face images acquired would be of arbitrary angles and varying illumination and other non-ideality factors. In such cases, reconstruction of frontal faces from the arbitrary pose variations would result in loss of information due to self-occlusions caused by largeangle, non-frontal pose variations. This results in a poor-quality frontal image which effects the 
match performance. A different approach to this problem is the use of 3D facial models created from 2D images which can be used to generate non-frontal images from frontal views for performance evaluation [50]. Another approach is generating a morphable model of 3D faces that represents face-specific information extracted from datasets of 3D scans [51].

\subsubsection{Texture-based Approaches}

Texture plays an important role for analysis of many types of images. In an image, the textured area includes many features like variations in distribution of intensity, noise, surface illumination, frequency response, and position of the camera. Texture-based region descriptors can be very useful in recognizing faces and facial expressions, detecting faces and different facial components. The local binary pattern (LBP) texture method has proved to be an excellent technique can be utilized in real-time applications [52]. This is because of its computational simplicity and robustness to illumination changes, unlike other methods. Thus, it has been shown to be advantageous over appearance-based approaches.

\section{Local Binary Patterns (LBP)}

The LBP texture analysis operator is defined as a gray-scale invariant texture measure. For each pixel in an image, a binary code is produced by thresholding its value with the value of the center pixel. A facial image is divided into numerous small windows and the histogram of LBP in each window is calculated. Local primitives codified by these bins include different types of curved edges, spots, flat areas etc. [52]. The image pixels of a 3x3 neighborhood or region are used in extracting these LBP features [2]. The basic version of the LBP operator considers only the 8 neighbors of a pixel. Later, the operator has been extended to consider different neighborhood sizes. Multiscale LBP is an extension to basic LBP. In general, for the operator represented as LBP $\mathrm{P}, \mathrm{R}$ refers to a neighborhood size of $\mathrm{P}$ equally spaced pixels on a circle of radius $\mathrm{R}$ that form a circularly symmetric neighbor set [52]. The number of bins in the histogram include 8 and $2 \mathrm{P}$ for LBP and MLBP respectively. 


\begin{tabular}{|l|l|l|}
\hline 180 & 176 & 168 \\
\hline 179 & 175 & 170 \\
\hline 169 & 174 & 170 \\
\hline
\end{tabular}

Example

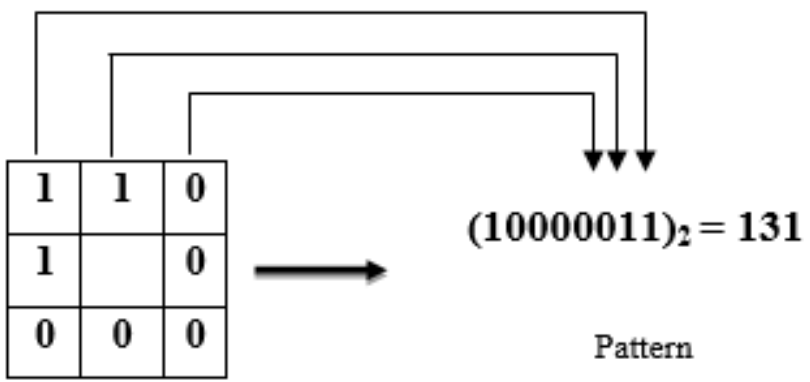

Threshold

Figure 2.7 Illustration of LBP with an example

\subsection{Performance Evaluation Methods}

In general, face recognition is a process that is performed using two different sets of images gallery and probe. In biometric face recognition systems, there are different modes of operations to test the identity of an individual. Major functionalities of face recognition systems are discussed in the following sections.

\subsubsection{Verification}

Verification is a one-to-one matching scenario in which the identity of an individual is verified over each record in the data set. Verification can otherwise be defined as authentication, which answers the question "Is the person who she/he claims to be?". In the process of verification, the identity of an individual is defined based on the threshold value, i.e. if the match score value of the image is greater than the threshold the image is authenticated and is considered genuine otherwise it is considered an imposter.

A desired facial recognition application possesses a low false accept rate (FAR) and high genuine accept rate (GAR) to achieve a small number of subjects which are falsely accepted as genuine and maximum number of genuine cases. 


\subsubsection{Identification}

Identification is a one-to-many matching scenario in which the identity of an individual is determined. Identification is quite different than verification, in which the test image is matched over the set of $\mathrm{n}$ images in the gallery, and generates similarity scores which determines the best match for the image. Identification is further classified into two different cases as: -

(I) Closed set identification, in which the probe image is matched over the available gallery set where there is at least one best match in the gallery.

(II) Open set identification, in which all the probe images may not have true matches in the gallery. This is also determined as the watch-list case of identification.

The identification performance of a facial recognition system is estimated by the percentage of rank 1 accuracy of the match score values of the subjects.

\subsubsection{Cumulative Match Characteristic Curves}

For a biometric system, as discussed earlier, the two major tasks are identification and verification. Verification is a one-to-one matching problem whereas identification is a one-to-many matching problem which is much harder when compared to verification. Cumulative Match Characteristic Curves (CMC) can be defined as a rank based metric. CMC curves serve as a significant performance evaluation measure of identification in face recognition.

CMC curve gives probabilities of recognizing an individual depending on how similar their biometric measurements are to another individual's biometric measurements [53]. In CMC curves, the plot includes all the possible ranks and the probability of identification is $100 \%$ at the highest rank. This occurs in a closed set identification where every input is in the database otherwise it would be an open set identification test. 


\section{Chapter 3 Experimental Data}

Experimental data is essential to biometrics research. As mentioned earlier, there are different types of facial image datasets. For this research effort, non-frontal 2D face images are created using 3D image files, with a range of angular variations in pitch, yaw, and coupled pitch/yaw direction. These non-frontal images are then pose-corrected using two techniques:

1. An open source software called MeshLab is used to create manually pose-corrected images with artifacts (occlusions, lower-resolution regions) like automated pose-corrected images

2. Pose-corrected images created using commercial software, Cognitec FaceVACs DB Scan.

The process of generation of these images is explained in the following sections of this chapter.

\subsection{Data set Description}

West Virginia University has conducted several biometric data collection projects since 2008. The facial images used in this project were part of the 2013 BioCOP data collection acquired using a Canon EOS 5D Mark III digital camera with a Canon Electro-Focus (EF) 70-200mm f/2.8L Image Stabilization (IS) III Ultrasonic Motor (USM) telephoto zoom lens. The 3D images were acquired using 3dMDface system manufactured by 3dMD [54].
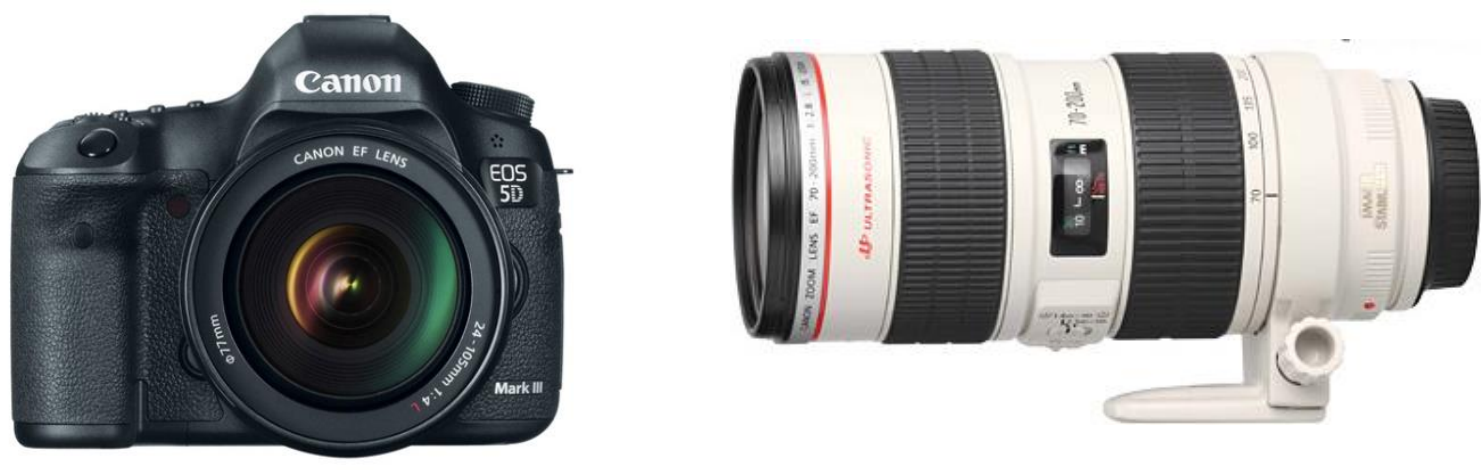

Figure 3.1 Canon EOS 5D Mark III camera and Canon EF 70-200mm f/2.8L IS III USM telephoto zoom lens 
Table 3.1 Image specifications in Dataset

\begin{tabular}{|c|c|}
\hline Image Format & JPG \\
\hline Back Ground & Neutral Gray \\
\hline Spatial Resolution & $72 \mathrm{dpi}$ \\
\hline Exposure Time & $1 / 60 \mathrm{sec}$ \\
\hline Focal Length & $135 \mathrm{~mm}$ \\
\hline Color Representation & sRGB \\
\hline Raw Image Resolution & $5616 \times 3744$ \\
\hline Cropped SAP 50 Image Resolution & $3300 \times 4400$ \\
\hline Cropped SAP 51 Image Resolution & $2400 \times 3200$ \\
\hline
\end{tabular}

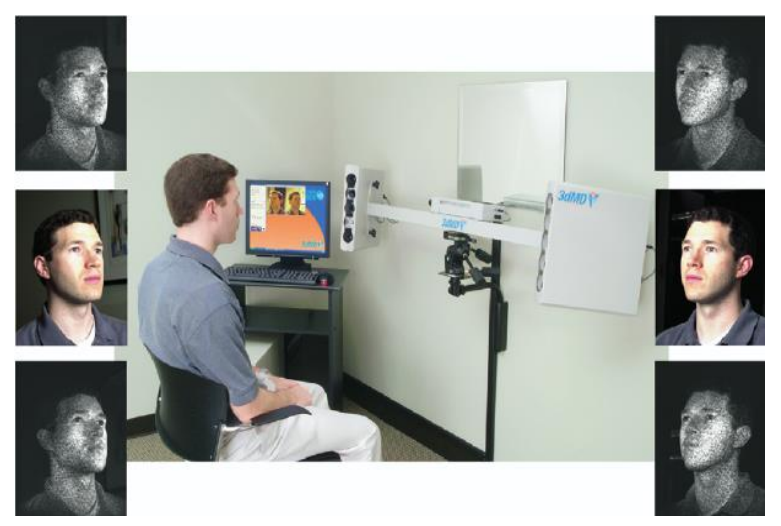

Figure 3.2 3dMD Face System [54]

The data collection has been performed in two sessions with a minimum separation of two months in which the images are captured twice for each participant in one session. The captured images in the collection include digital camera images with angular variations of $0^{\circ}, \pm 45^{\circ}$, and $\pm 90^{\circ}$ along with two frontal images each with color card and random identification number respectively. Images in the data collection are acquired at 2 meters against a neutral gray background with threepoint tungsten lighting to eliminate shadows and hot spotting. In this experiment, only the frontal images of subjects are used to perform the identification test which are 8 or 16 images per subject depending on the sessions the subject has participated in. Parameters such as spatial resolution, 
focal length and exposure time etc. are maintained constant throughout the process of acquisition for the 1079 subjects belonging to a wide range of demographics. In this project of the 1079 subjects who have participated in the collection for one or two sessions, 100 subjects were selected from different ethnicities and age groups.

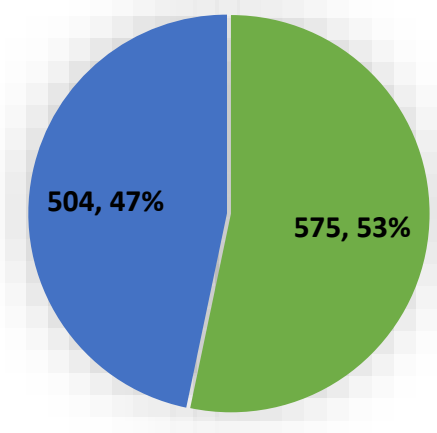

(a)

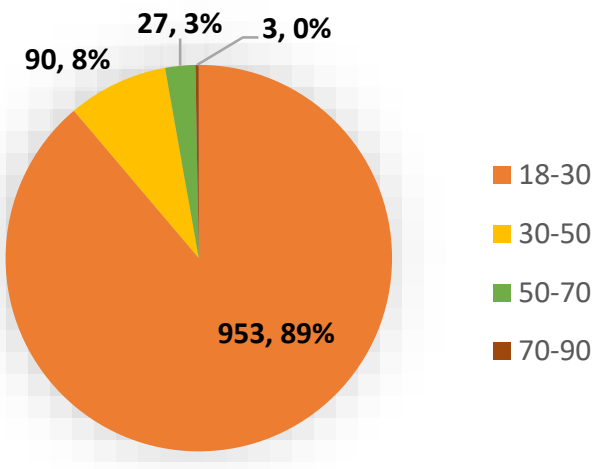

(b)

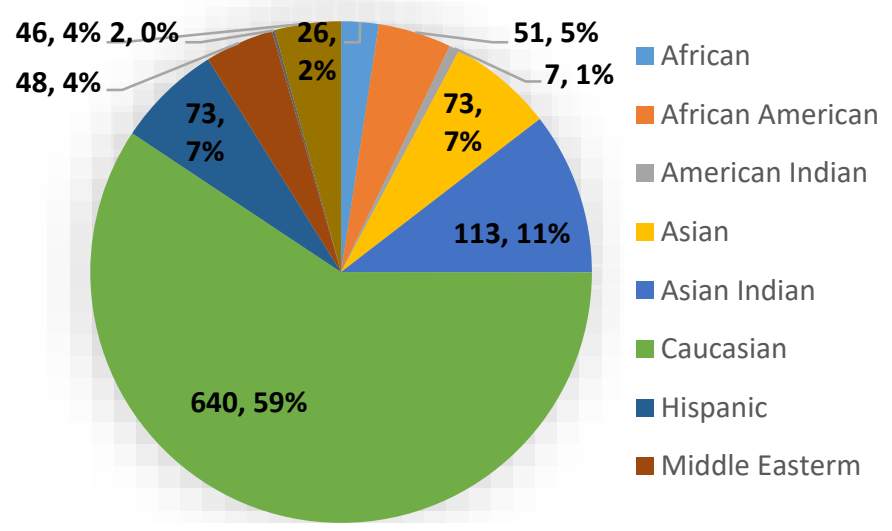

(c)

Figure 3.3 Pie chart Representation of Gender, Age, Ethnicity of the Total Data Collected. (a) Gender, (b) Age, (c) Ethnicity 


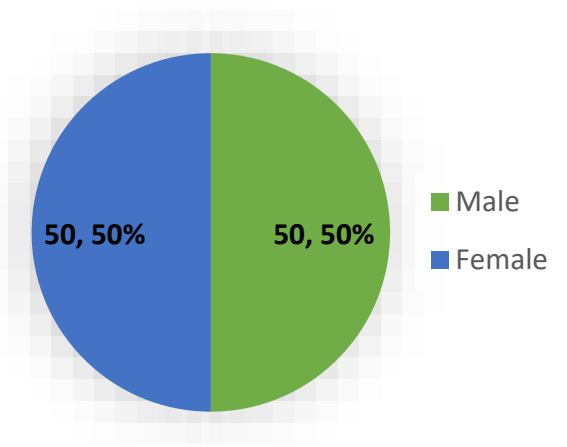

(a)

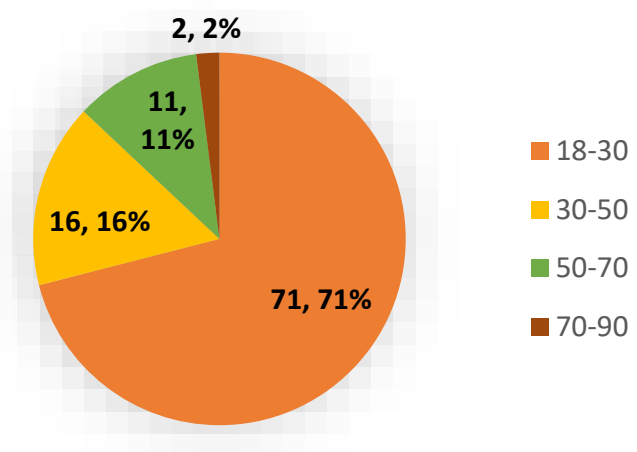

(b)

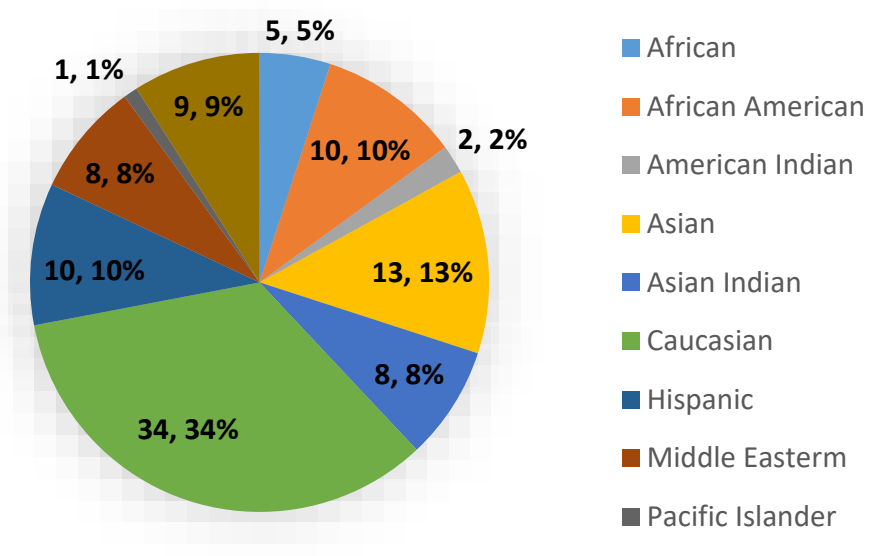

(c)

Figure 3.4 Pie chart Representation of Gender, Age, Ethnicity of the Selected Data in the Project. (a) Gender, (b) Age, (c) Ethnicity

The 2D images are post processed to generate cropped images compatible with ANSI/NIST SAP 50/51 mugshot composition standards [55]. The 3D images captured using the 3dMD system are post processed to generate 3D data files compatible with a variety of rendering tools, including: .OBJ, .PLY, .STL, .TSB and .X3D. In this project, .OBJ and. PLY files have been used to generate non-frontal pose images using 3D models, and then pseudo-pose corrected images were generated using MeshLab, which is an open source software. Using NeuroTechnology MegaMatcher SDK (Software Development Kit) v5.0 and Cognitec FaceVACs Matcher the matching performance of 
the non-ideal images has been evaluated and compared to the matching performance of frontal images and non-pose-corrected images.

As already mentioned, 100 subjects (50 Male and 50 Female) have been selected out of the original database of 1079 participants. Of these 100, some subjects have participated only in one session whereas some have participated in both the sessions. Subjects who have participated only in one session have 8 images while subjects participating in both the sessions have 16 images which forms a frontal gallery of 1203 images.

\subsection{Creation of Pseudo-Pose-Corrected Images}

The subset of facial images taken from the 2013 dataset was pre-processed prior to matching experiments. The files associated with 3D images have 5 sub-folders with different file formats. For this effort, only the '.OBJ', '.PLY' files are used due to their compatibility with open-source software tools. The 3D files are converted to 2D files with varying roll and yaw angles, as well as regions with varied polygon count, using MeshLab.

The processes in which the 3D files are pre-processed to build a dataset of non-frontal 2D images is explained as follows:

- First .OBJ files of the image in the 3D files of the data set are read into Mesh lab and next the PLY files are read into Mesh lab. These two put together resemble a mesh overlap as shown in Figure 3.5 (a).

- Next the image is set to normal by adjusting the Field of View (FOV) to 'Ortho' in Mesh lab.

- To find the performance of pose corrected images, images with varying pose angles are then generated as shown in Figure 3.5 (b). In this project the poses are varied from $\mathbf{1 0}^{\circ}$ to $\mathbf{6 0}^{\circ}$ with an interval of $10^{\circ}$.

- The rotated images possess occluded regions which are purposefully distorted by reducing the polygon count at those areas from $\mathbf{2 5 \%}$ to $\mathbf{5 0 \%}$ with a $\mathbf{2 5 \%}$ increase for each operation. 
- The polygon count of the images is reduced using Quad Edge Collapse Decimation Filter in Mesh lab as shown in Figure 3.5 (e).

- This polygon count reduced images are pose corrected images in $3 \mathrm{D}$, which are further exported from Mesh lab as 2D images for match performance evaluation.

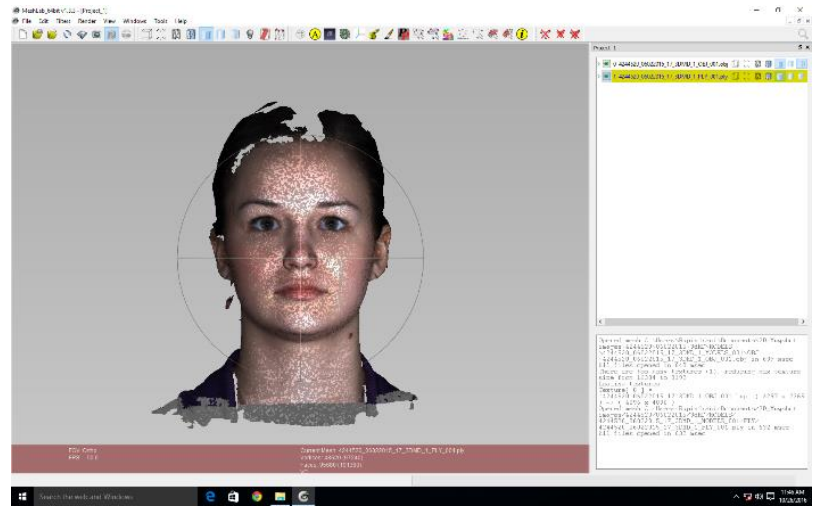

(a)

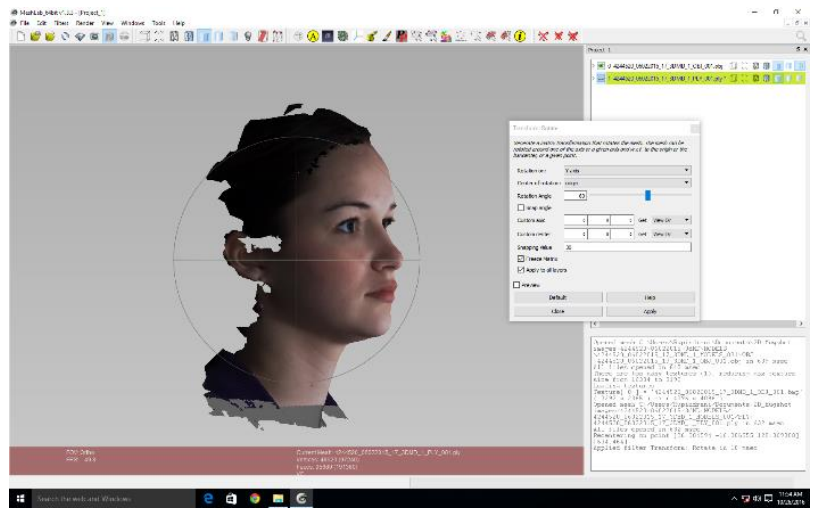

(c)

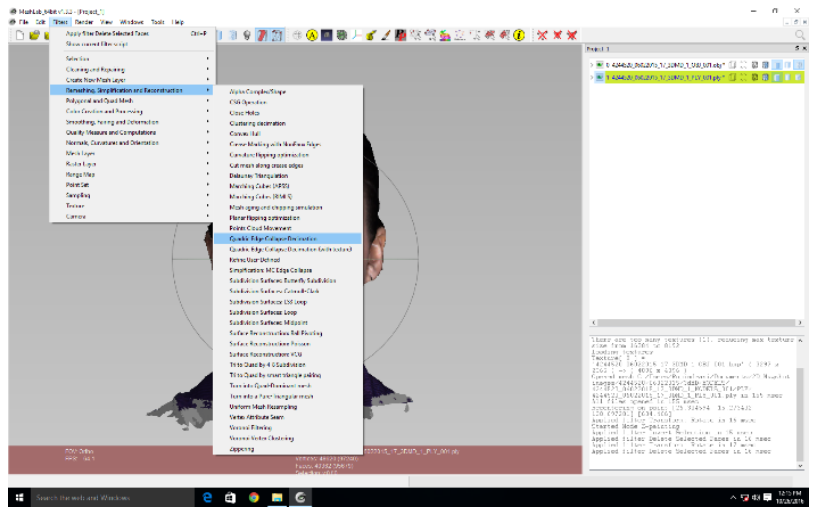

(e)

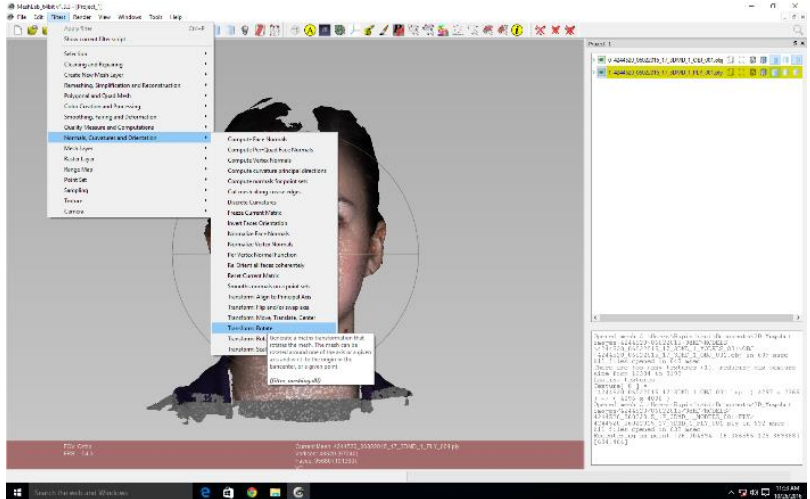

(b)

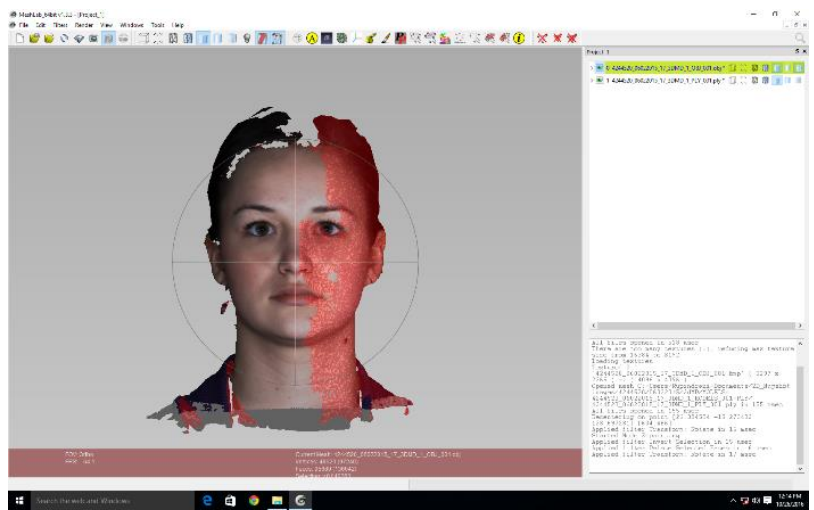

(d)

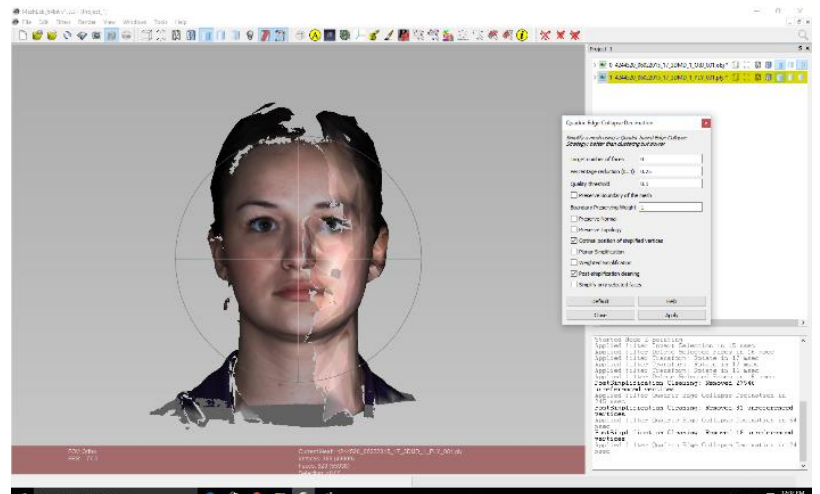

(f) 
Figure 3.5 Illustration of steps in generation of pseudo pose corrected image from 3D image (a) Reading 3d files into MeshLab (b), (c) Applying Transform rotate filter (d) Marking the occlusion regions (e), (f) Applying 'Quadric Edge Collapse Decimation'

\subsubsection{Angular Rotations}

In this project, three different types of non-frontal images are generated which include either pitch rotations, yaw rotations, or both pitch and yaw rotations. As discussed earlier, pose variation is one of the major challenges in face recognition which affects the performance of a face recognition system. In this work, match performance of non-frontal images with varying poses and pseudo pose images generated using $3 \mathrm{D}$ image scans is evaluated to assess the performance of recognition using non-ideal images.

The rotations of pitch, roll, and yaw angles are as shown in the Figure 3.6

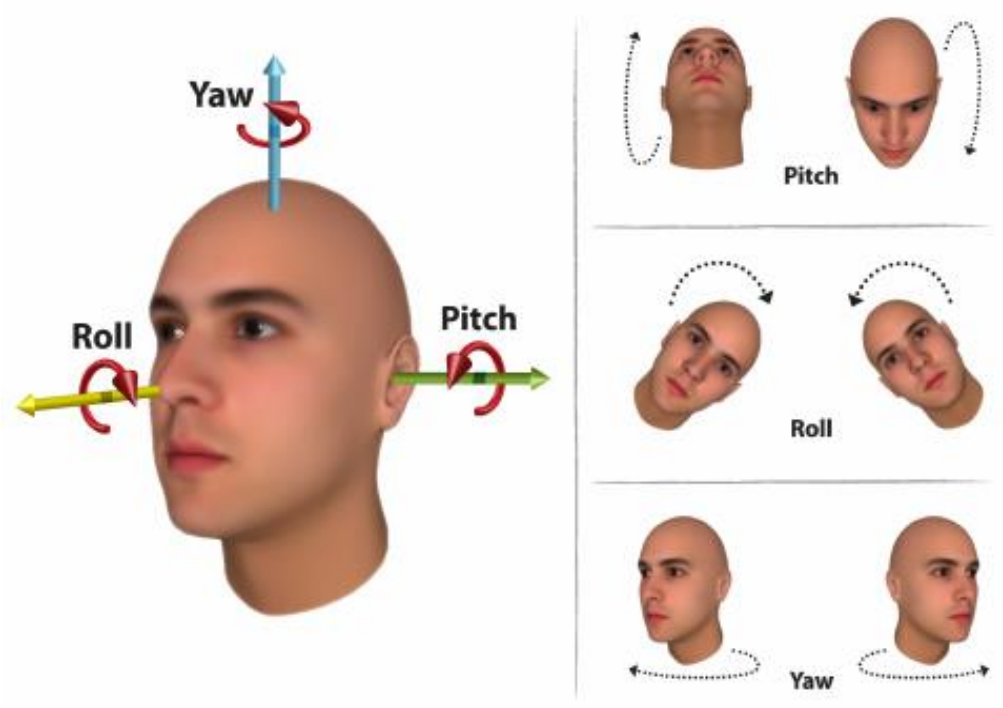

Figure 3.6 Orientation of head in terms of Pitch, Roll and Yaw angular rotations [56]

\section{Pitch Images}

Pitch angles are rotated above or below the horizontal axis in a 3-dimensional plane. The rotations of human head in pitch can be seen in Figure 3.6. Examples of pose-varying and pseudo-pose- 
corrected pitch images generated from 3D scans using the method described in section 3.2 are shown in Figure 3.7. Pitch angle was varied from $10^{\circ}$ to $60^{\circ}$ and from $-10^{\circ}$ to $-60^{\circ}$ with an increment of $10^{\circ}$ in each case.

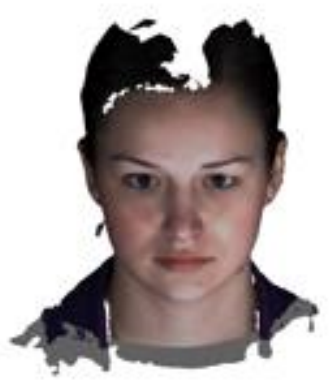

$20^{\circ}$ Pitch

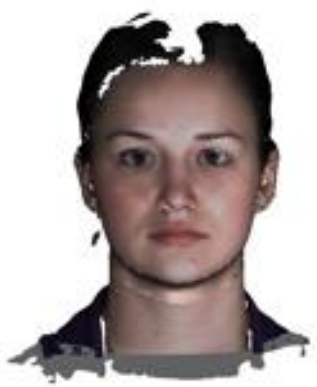

Pseudo-pose-corrected $20^{\circ} \mathrm{Pitch}$ at $25 \%$ Resolution Reduction

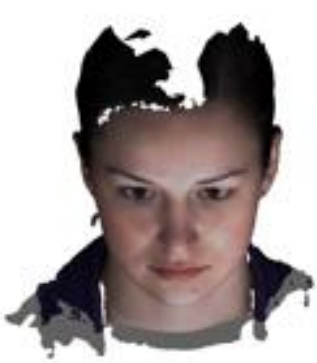

$30^{\circ}$ Pitch

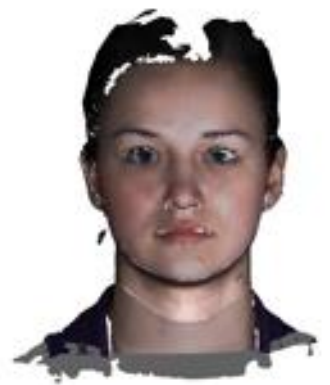

Pseudo-pose-corrected $30^{\circ}$ Pitch at $25 \%$ Resolution Reduction

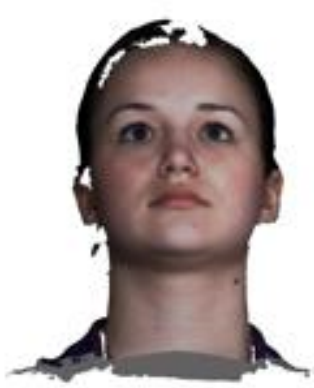

$-20^{\circ}$ Pitch
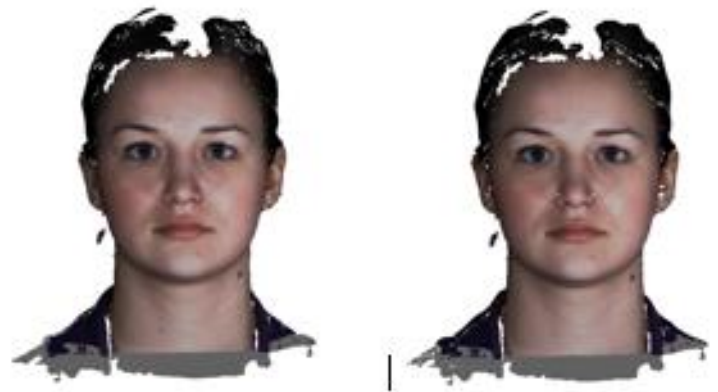

Pseudo-pose-corrected - Pseudo-pose-corrected $20^{\circ}$ Pitch at $25 \%$ Resolution Reduction

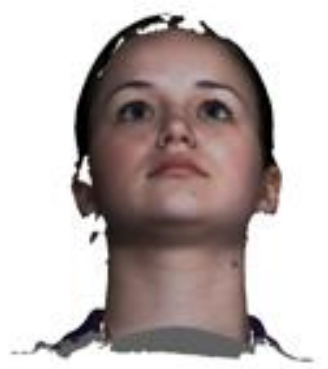

$-30^{\circ}$ Pitch $30^{\circ}$ Pitch at $25 \%$

Resolution Reduction

Figure 3.7 Pitch Varying Pose and Pseudo Pose Corrected Images

\section{Yaw Images}

Yaw angles are rotated left or right about the vertical axis in a 3-dimensional plane. Considering the bilateral symmetry of human face, yaw rotations only in positive variation were generated in this work. Examples of yaw rotations applied in this work are as shown in Figure 3.8. Yaw angle variations are from $10^{\circ}$ to $60^{\circ}$ with $10^{\circ}$ increment in each case. 


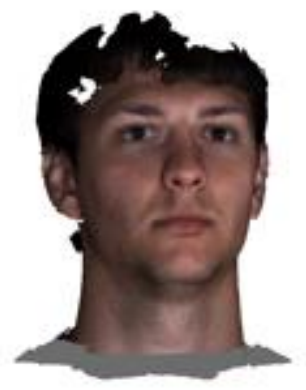

$10^{\circ}$ Yaw

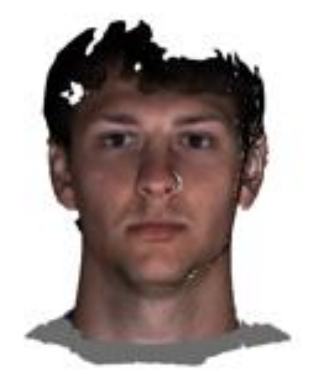

Pseudo-pose-corrected $10^{\circ}$ Yaw at $25 \%$ Resolution Reduction

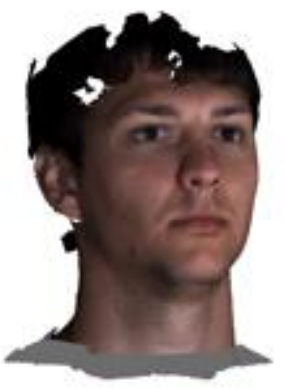

$20^{\circ}$ Yaw

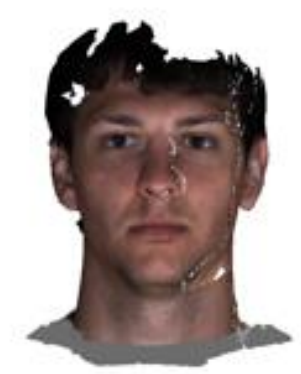

Pseudo-pose-corrected $10^{\circ}$ Yaw at $25 \%$ Resolution Reduction

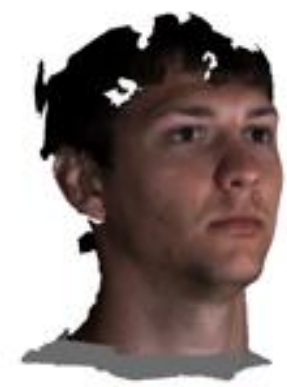

$30^{\circ}$ Yaw

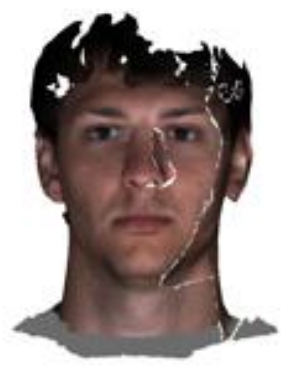

Pseudo-pose-corrected $10^{\circ}$ Yaw at $25 \%$ Resolution Reduction

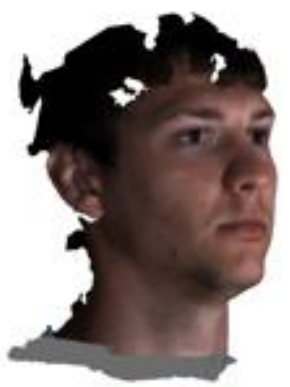

$40^{\circ}$ Yaw

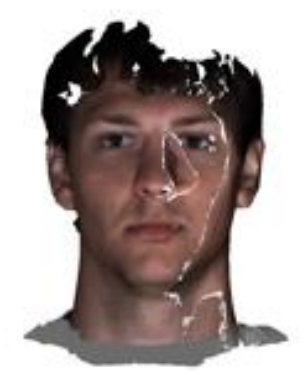

Pseudo-pose-corrected $10^{\circ}$ Yaw at $25 \%$ Resolution Reduction

Figure 3.8 Yaw Varying Pose and Pseudo Pose Corrected Images

\section{Pitch and Coupled}

A combination of pitch and yaw angle rotation is implemented in this project. Four different angular variations are applied to images, which are Pitch $30^{\circ}$ Yaw $15^{\circ}$, Pitch $30^{\circ}$ Yaw $30^{\circ}$, Pitch $30^{\circ}$ Yaw $45^{\circ}$ and Pitch $30^{\circ}$ Yaw $60^{\circ}$. Similarly Pitch $-30^{\circ}$ Yaw $-15^{\circ}$, Pitch $-30^{\circ}$ Yaw $-30^{\circ}$, Pitch $30^{\circ} \mathrm{Yaw}-45^{\circ}$ and Pitch $-30^{\circ} \mathrm{Yaw}-60^{\circ}$ are also applied. Sample images with variations in pitch and yaw angles are shown in Figure 3.9. 


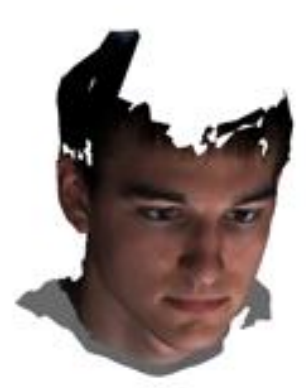

$30^{\circ}$ Pitch, $15^{\circ}$ Yaw

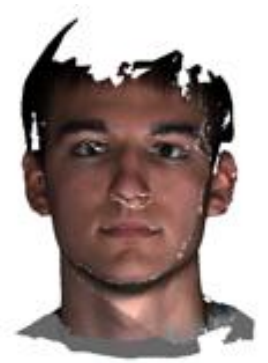

Pseudo-pose-corrected $30^{\circ}$ Pitch, $15^{\circ}$ Yaw at $25 \%$ Resolution Reduction

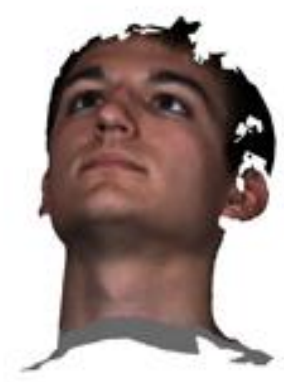

$-30^{\circ}$ Pitch, $-15^{\circ}$ Yaw

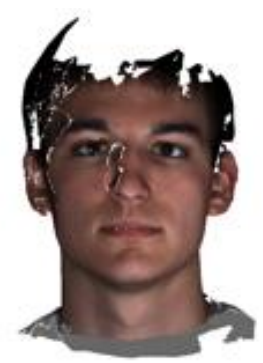

Pseudo-pose-corrected Resolution Reduction

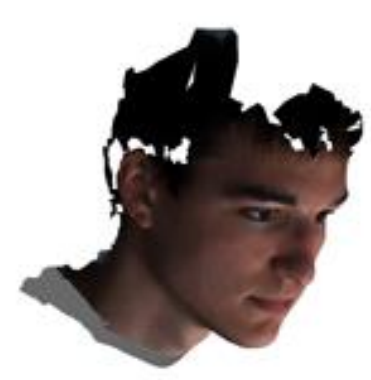

$30^{\circ}$ Pitch, $45^{\circ}$ Yaw

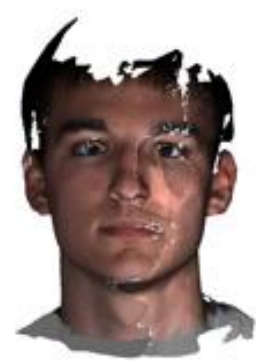

Pseudo-pose-corrected Pseudo-pose-corrected $30^{\circ} \mathrm{Pitch}, \mathbf{4 5}^{\circ} \mathrm{Yaw}$ at $25 \%-30^{\circ} \mathrm{Pitch},-\mathbf{4 5}^{\circ} \mathrm{Y}$ aw at $25 \%$

Resolution Reduction

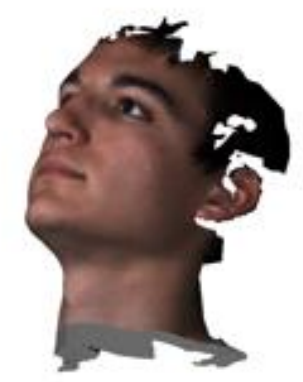

$-30^{\circ}$ Pitch, $-45^{\circ}$ Yaw

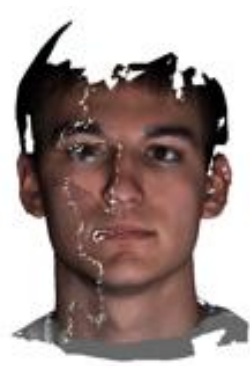

Resolution Reduction

Figure 3.9 Pitch and Yaw Varying Pose and Pseudo Pose Corrected Images

These non-frontal images together form a non-ideal image data-base with a total of 10,400 images, which includes:

- 1200 pose varying pitch rotation images $\left(10^{\circ}\right.$ to $60^{\circ},-10^{\circ}$ to $60^{\circ}$ with $10^{\circ}$ increment $)$

- 600 pose varying yaw rotation images $\left(10^{\circ}\right.$ to $60^{\circ}$ with $10^{\circ}$ increment)

- 800 pose varying pitch and yaw rotation images (Pitch $30^{\circ}$ Yaw $15^{\circ}$, Pitch $30^{\circ}$ Yaw $30^{\circ}$, Pitch $30^{\circ}$ Yaw $45^{\circ}$ and Pitch $30^{\circ}$ Yaw $60^{\circ}$, Pitch $-30^{\circ}$ Yaw $-15^{\circ}$, Pitch $-30^{\circ}$ Yaw $-30^{\circ}$, Pitch $30^{\circ}$ Yaw $-45^{\circ}$ and Pitch $-30^{\circ}$ Yaw $-60^{\circ}$ ).

It also consists of pseudo pose corrected images generated in MeshLab with varying polygon count $(25 \%, 50 \%$ and $75 \%)$ at the occluded regions. In total, there are 3600 pseudo pose corrected pitch 
images, 1800 pseudo pose corrected yaw images and 2400 pseudo pose corrected pitch and yaw rotation images. The dataset description is given in the Table 3.2.

Table 3.2 Description of dataset generated in this study

\begin{tabular}{|c|c|}
\hline \multicolumn{2}{|c|}{$\begin{array}{l}2013 \text { FBI Biometric Collection Project } \\
\text { NIST SAP 50/51 Face images captured from } 1079 \text { participants (444 repeat visits) }\end{array}$} \\
\hline Sessions & 2 \\
\hline Frontal Gallery Images & $\begin{array}{c}\mathbf{1 2 0 3} \\
\text { (Subjects participating in } 2 \text { sessions have } 16 \text { images } \\
\text { each. Subjects participating in only one session have } 8 \\
\text { images) }\end{array}$ \\
\hline $\begin{array}{l}\text { Pitch Rotation Pose } \\
\text { Varying Images }\end{array}$ & $\begin{array}{c}\mathbf{1 2 0 0} \\
-60 \text { to }+60 \text { degrees of rotation in } 10 \text { degree increments }\end{array}$ \\
\hline $\begin{array}{l}\text { Yaw Rotation Pose } \\
\text { Varying Images }\end{array}$ & $\begin{array}{c}\mathbf{6 0 0} \\
10 \text { to } 60 \text { degrees in } 10 \text { degree increments (negative } \\
\text { angles not considered due to bilateral facial symmetry) }\end{array}$ \\
\hline $\begin{array}{l}\text { Coupled Pitch/Yaw } \\
\text { Rotation Pose Varying } \\
\text { Images }\end{array}$ & $\begin{array}{c}\mathbf{8 0 0} \\
\left(30^{\circ} / 30^{\circ}, 30^{\circ} / 15^{\circ}, 30^{\circ} / 45^{\circ}, 30^{\circ} / 60^{\circ},-30^{\circ} /-30^{\circ},-30^{\circ} /-15^{\circ}\right. \\
\left.-30^{\circ} /-45^{\circ},-30^{\circ} /-60^{\circ}\right)\end{array}$ \\
\hline $\begin{array}{l}\text { Pitch Rotation Pseudo } \\
\text { Pose Corrected Images }\end{array}$ & $\begin{array}{c}\mathbf{3 6 0 0} \\
-60 \text { to }+60 \text { degrees of rotation in } 10 \text { degree increments }\end{array}$ \\
\hline $\begin{array}{l}\text { Yaw Rotation Pseudo } \\
\text { Pose Corrected Images }\end{array}$ & $\begin{array}{c}\mathbf{1 8 0 0} \\
10 \text { to } 60 \text { degrees in } 10 \text { degree increments (negative } \\
\text { angles not considered due to bilateral facial symmetry) }\end{array}$ \\
\hline $\begin{array}{l}\text { Coupled Pitch/Yaw } \\
\text { Pseudo Pose Corrected } \\
\text { Images }\end{array}$ & $\begin{array}{c}\mathbf{2 4 0 0} \\
\left(30^{\circ} / 30^{\circ}, 30^{\circ} / 15^{\circ}, 30^{\circ} / 45^{\circ}, 30^{\circ} / 60^{\circ},-30^{\circ} /-30^{\circ},-30^{\circ} /-15^{\circ},\right. \\
\left.-30^{\circ} /-45^{\circ},-30^{\circ} /-60^{\circ}\right)\end{array}$ \\
\hline
\end{tabular}




\subsection{Creation of Pose-Corrected Images Using Cognitec DB Scan}

Cognitec DB Scan v5.0 is a commercial software from Cognitec and is designed for face biometric applications. DBScan is an automated software for face enrollment, identification, and verification. The components of FaceVACs DBScan include: database Enrollment, database identification, database verification, database examiner, and case management. Each has a unique functionality which purportedly aids in better performance of the face recognition process. DBScan also has an algorithm designed for pose normalization of 3D models which generates a frontal face image from a non-frontal, arbitrary pose image.

Pose varying images generated from 3D models are read into the DBScan Examiner and then the $3 \mathrm{D}$ pose normalization algorithm is applied to produce the pose corrected images. The process of pose normalization in DBScanner is as shown in the Figure 3.10

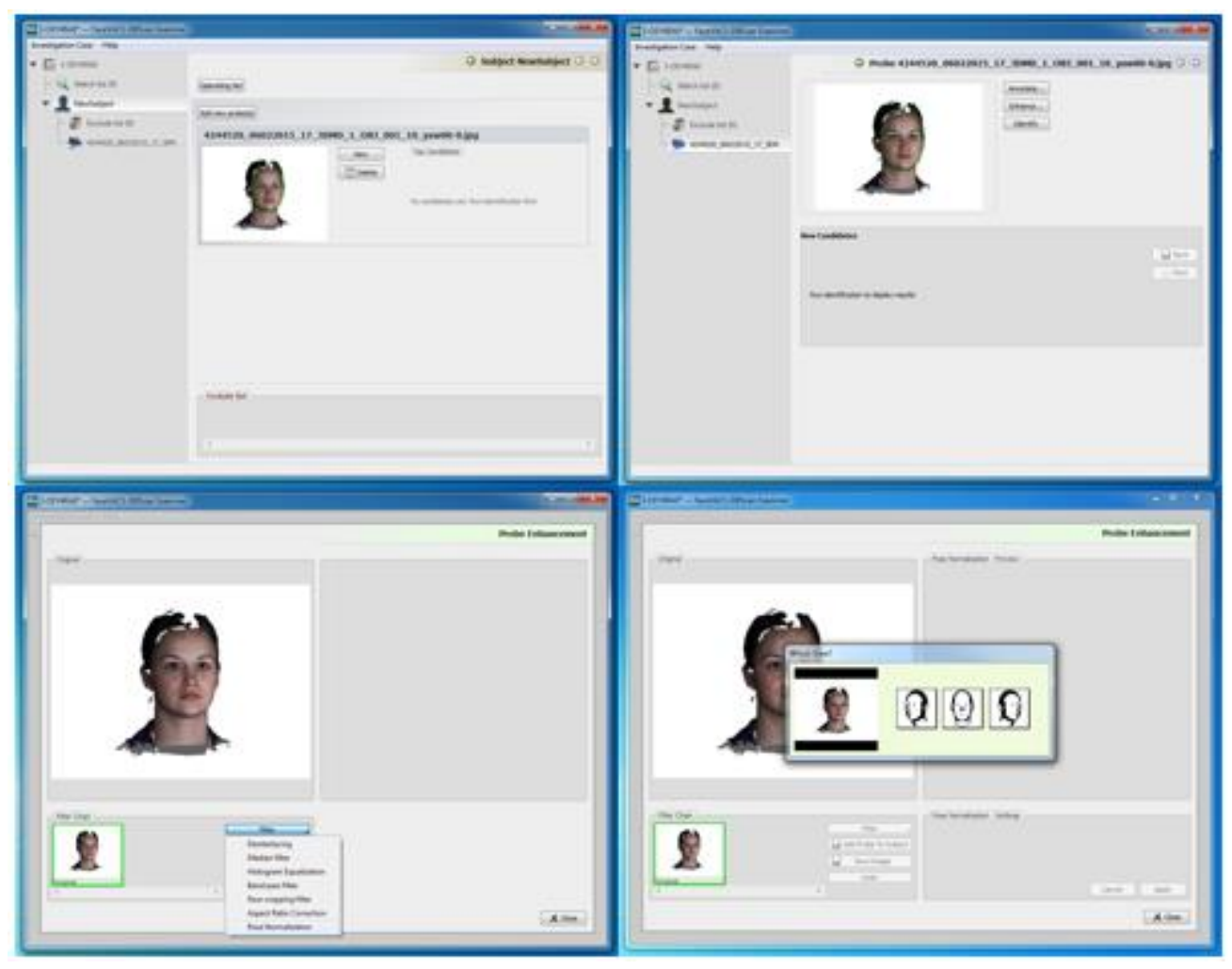

(a) 


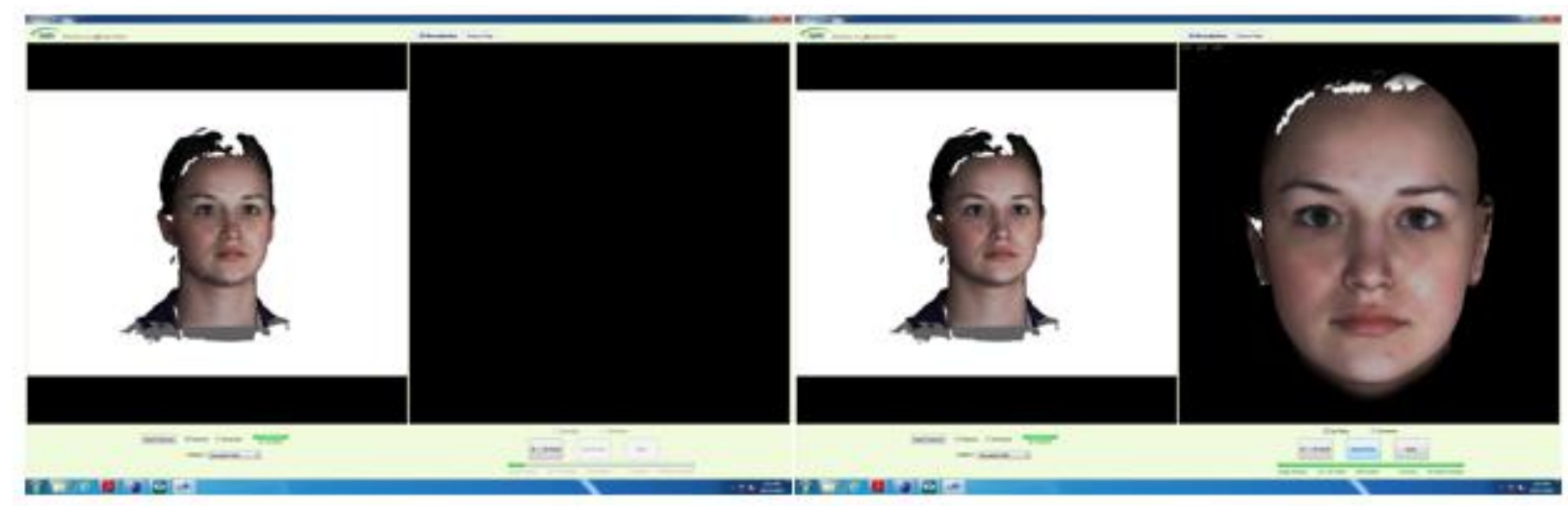

(b)

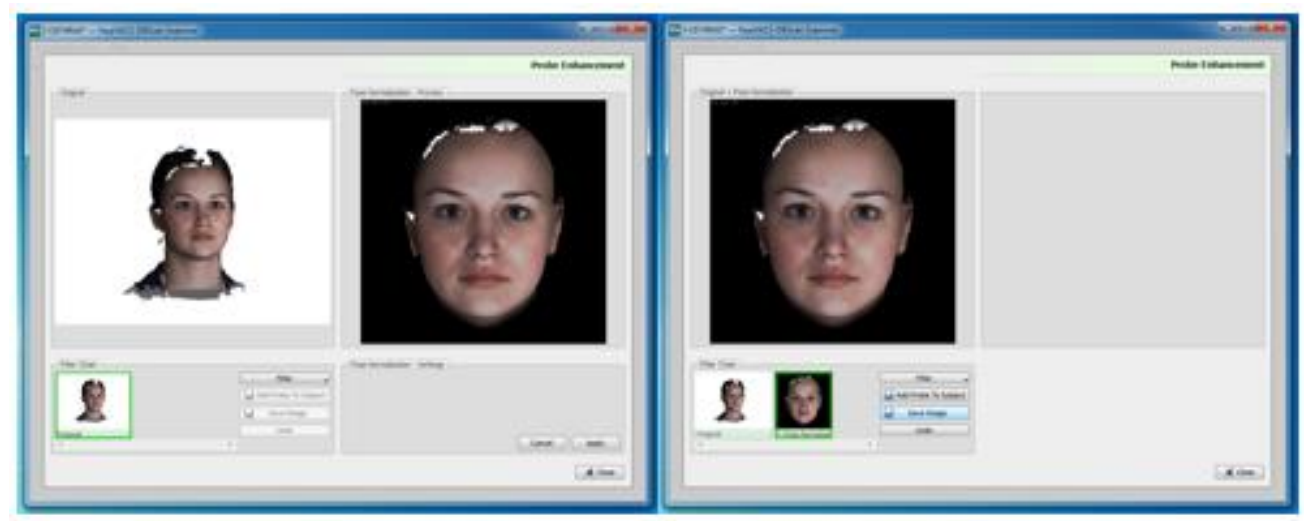

(c)

Figure 3.10 Steps in Generation of Pose Normalized Images from DB Scanner (a) Steps to read a probe image into Cognitec DB Examiner, (b) Applying the pose normalization filter in DB Scanner, (c) Saving the generated normalized image.

- Initially pose-varying images are enrolled into the DB Scan using DB Scan Examiner tool. The desired probe image is selected and is enhanced using the Enhance option.

- In enhancement, there are different filters like Median filter, Histogram Equalization, Band pass filter and Pose Normalization. To perform pose correction, Pose Normalization filter is applied on pose-varying probe images.

- After selecting the pose normalization filter the desired pose of the final image is chosen as shown in Figure 3.10 (a). 
- Pose Normalization filter extracts the features of the face and converts the $2 \mathrm{D}$ image to a 3D model to generate a neutral pose of the probe image as shown in Figure 3.10 (b).

- After applying the filter the resultant image can be saved as shown in Figure 3.10 (c). 


\section{Chapter 4 Face Matching Experiments}

The main goal of this project is to evaluate the matching performance of $2 \mathrm{D}$ non-ideal posecorrected and pseudo-pose-corrected images generated using 3D models using commercial matchers with and without pose correction toolboxes. The process of configuring the matchers and running iterations of match score generation for probe inputs of frontal images, non-pose-corrected images, and pose-corrected images (both with manually generated and auto-generated pose correction) against a gallery of frontal images are explained in detail in this chapter.

\subsection{Generation of Match Scores}

A similarity score is a mathematical metric used to determine the similarity between images. During the matching, the probe set of images, which are the query images, are compared with the gallery set of images, which are the available images in the dataset, and a similarity score is generated. The extent of similarity between two images can be determined by comparing the features of the probe image against all the images in the gallery set. In general, the image feature information is extracted and stored in the form of a template, which is later used for identification of an individual from the available set of data. Here, two commercial matchers, NeuroTechnology MegaMatcher SDK (Software Development Kit) v5.0 and Cognitec FaceVACs SDK v9.0 were used in matching experiments to generate the similarity scores.

\subsubsection{Commercial Matchers}

This section describes the configuration of the commercial matchers used to generate the match score statistics. NeuroTechnology MegaMatcher SDK v5.0 with a JAVA user interface and Cognitec FaceVACs v9.0 with an API (Application Program Interface) implemented in a JAVA interface.

\subsubsection{MegaMatcher SDK Matching Software}

NeuroTechnology offers a multimodal SDK that includes various applications to meet the rising demand for biometric authentication. This SDK includes various components for individual biometric modalities, including VeriFinger, VeriEye, VeriLook and VeriSpeak for fingerprint, iris, 
facial and voice identification respectively. The relationship of these components within MegaMatcher is illustrated in Figure 4.1.

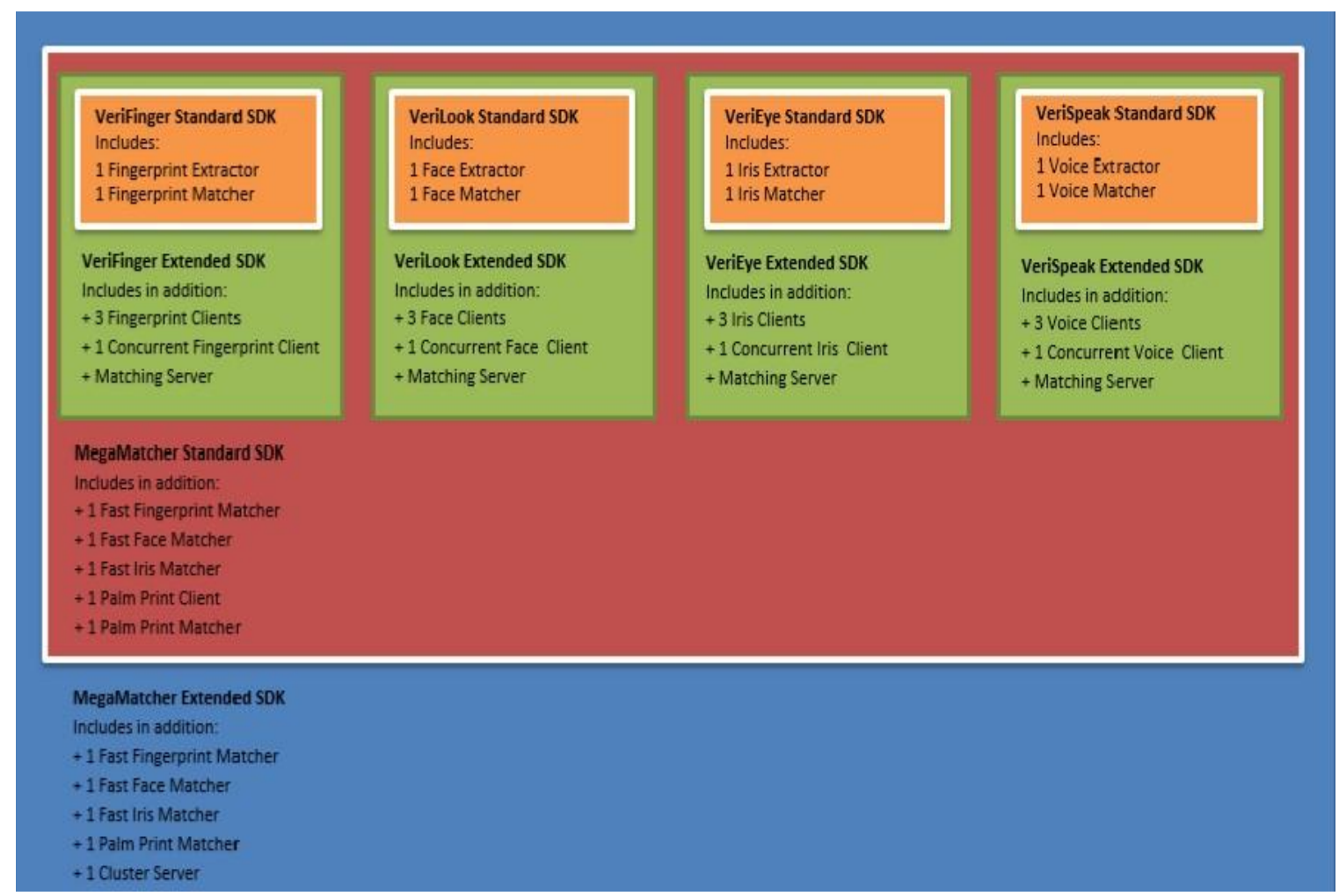

Figure 4.1 Schematic representation of NeuroTechnology Biometric SDK [57]

The SDK can be customized by the client as per the requirements of the application, with JAVA and .NET software platforms for the user to generate customized scripts using different programming environments.

The process of face matching in the NeuroTechnology Verilook SDK is illustrated in the flow diagram shown in Figure 4.2. It consists of two phases, extraction and identification. 


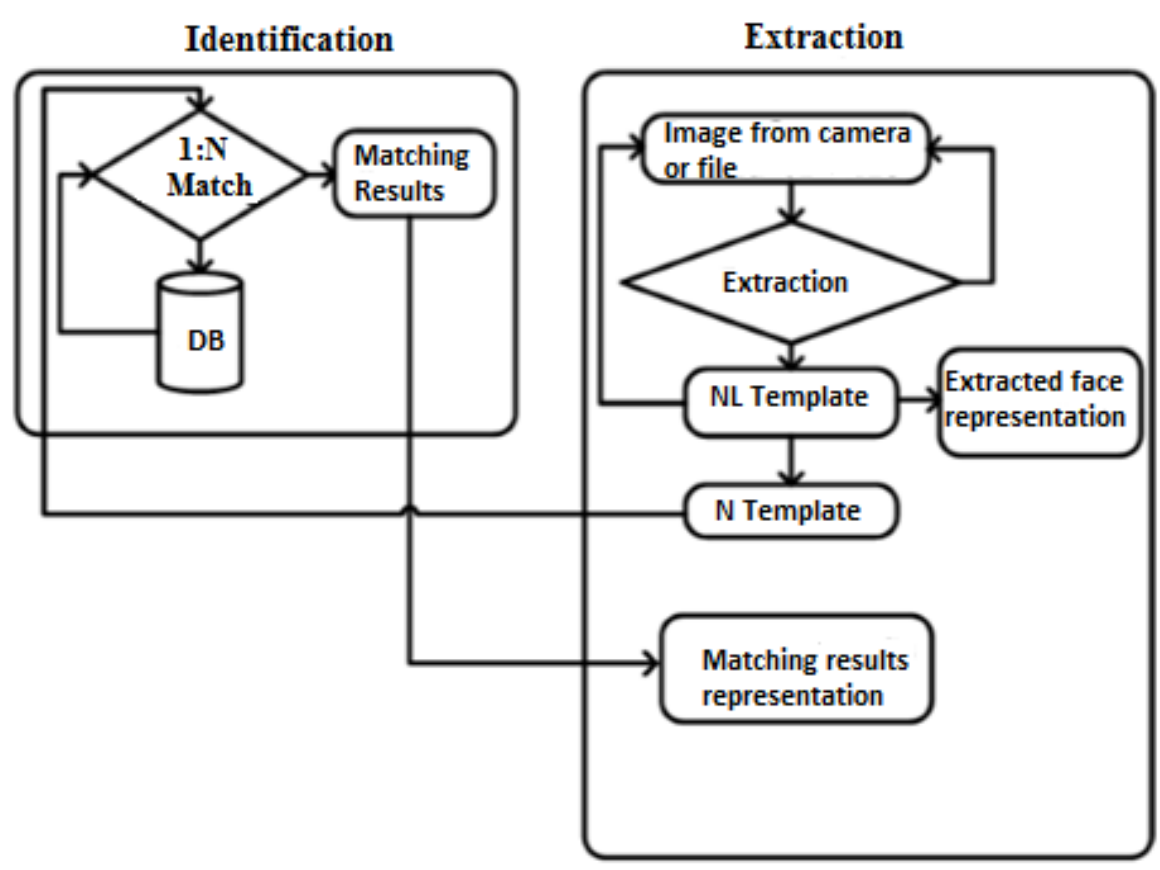

Figure 4.2 Flow Diagram of VeriLook Matching Mechanism [57]

An overview of the algorithm is as follows:

- Step 1: Raw images from the camera or pre-existing images files from a database can be given as input.

- Step 2: Feature extraction is performed in the extraction step and the template is created. 'NL template' is the terminology used by the software to denote a face template.

- Step 3: Extracted facial data for each facial image is extracted, and the set of templates created as NL templates are represented as N templates.

- Step 4: The testing procedure can be verification or identification, as per the requirement of the user application, and this is done on the NL templates created.

- Step 5: Matching results in this test are the similarity scores of the query image and the image in the data set. These results can be saved in desired formats. 


\subsubsection{Cognitec FaceVACs SDK Matching Software}

The Cognitec FaceVACs SDK is a software designed mainly for face biometric, and possessed functionalities such as face enrollment, identification, verification and face image quality assessment. The user has the freedom to customize in the software using different API's multiple programming languages such as:- C, C++, JAVA, .NET.

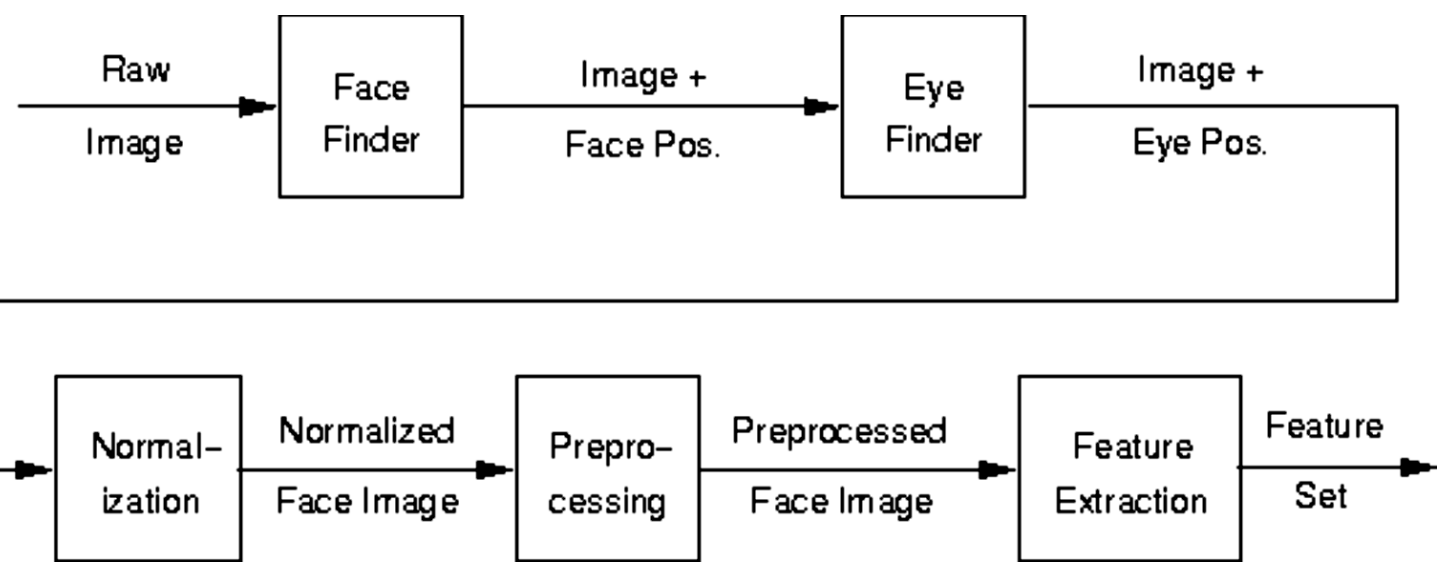

Figure 4.3 FaceVACs architecture: Feature set creation [58]

Figures 4.3 and 4.4 illustrate the mechanism of the feature set creation and the process of enrollment and verification using Cognitec FaceVACs Technology. The essential steps needed to create a feature set from raw images involve: Face Finder, Eye Finder, Normalization, Preprocessing, Feature Extraction. After this procedure, the software attains the feature set with information of the image and then follows the enrollment process. 


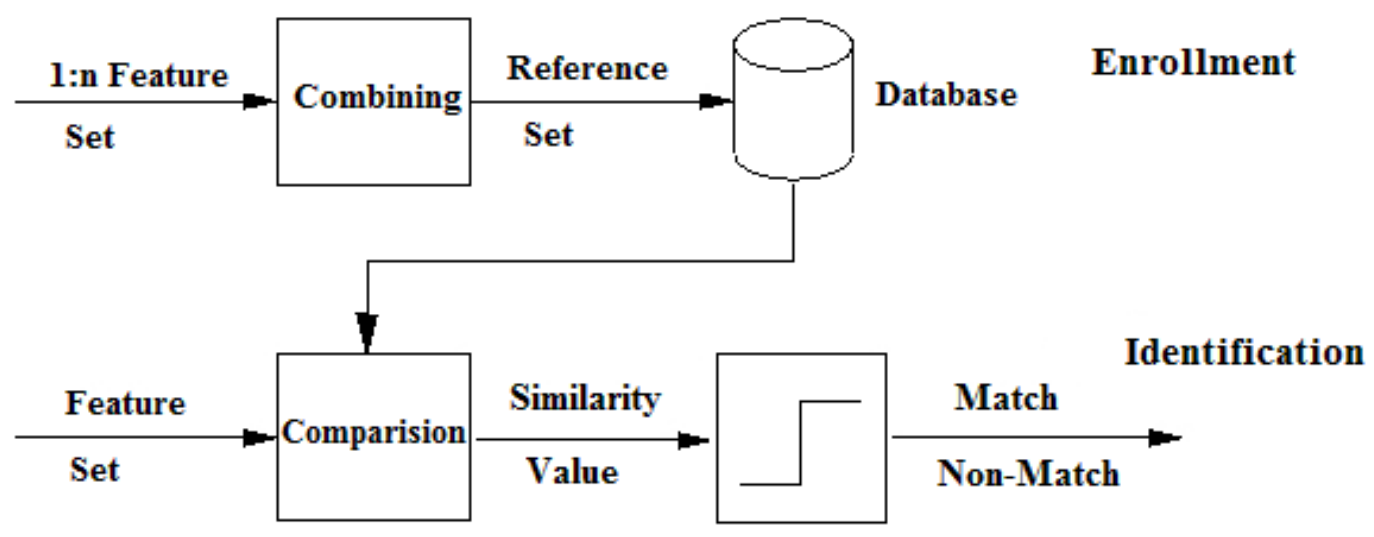

Figure 4.4 FaceVACs architecture: Enrollment and Identification [58]

In the enrollment process, the feature information of each image from the feature set is processed, which is used to generate a Face Identification Record (FIR) for each image. The set of FIR's generated are then compared over the reference set, and a score is produced in an order which defines the similarity between the images compared, and thus, a match or a non-match is determined. Both verification and identification tests can be performed using this software, and the threshold can be estimated based on the targeted FAR/FRR rates.

\subsection{Configuration of Matching Experiments}

\subsubsection{MegaMatcher SDK}

In this project, MegaMatcher is implemented using customized JAVA scripts to perform matching tasks to generate match scores. Table 4.1 lists the various attributes that have been used in this process. 
Table 4.1 Attributes of NeuroTechnology SDK used in this work [57]

\begin{tabular}{|c|c|}
\hline Attribute & Description \\
\hline NBiometricOperation & $\begin{array}{l}\text { A part of NBiometricEngine used in providing high level } \\
\text { operations such as template creation, enrollment, } \\
\text { identification or verification. }\end{array}$ \\
\hline NBiometricStatus & Enumerates biometric status values \\
\hline NBiometricTask & $\begin{array}{l}\text { Contains functions which create and modify biometric } \\
\text { engine tasks such as adding new subject or deleting existing } \\
\text { subject, retrieving biometric operation and calculating } \\
\text { statistics of a specific task etc. }\end{array}$ \\
\hline NFace & $\begin{array}{l}\text { A function for the biometric engine to deal with face } \\
\text { templates. These functions include creating a new face } \\
\text { object, retrieve the face image of a specified NFace object. }\end{array}$ \\
\hline NMatchingResult & $\begin{array}{l}\text { Used to retrieve information such as biometric connection, } \\
\text { ID, match score, subject etc. of a specific matching result. }\end{array}$ \\
\hline NMatchingSpeed & Defines rate of matching. \\
\hline NSubject & $\begin{array}{l}\text { Represents biometric information such as template, } \\
\text { matching results etc. related to a person. }\end{array}$ \\
\hline NBiometricClient & $\begin{array}{l}\text { Provides biometric connection function for the biometric } \\
\text { engine. Also provides the function for biometric data } \\
\text { capture and its transfer through various connections. }\end{array}$ \\
\hline NLicense & $\begin{array}{l}\text { Provides functionality for obtaining and releasing license. } \\
\text { Serves as an internal licensing service. }\end{array}$ \\
\hline
\end{tabular}




\subsubsection{Cognitec FaceVACs SDK}

The JAVA interface in Cognitec FaceVACs is utilized to generate a customized design for the identification tests in this project. Different attributes for face applications which have been used in this work are described in Table 4.2.

Table 4.2 Attributes of Cognitec FaceVACs used in this work [58]

\begin{tabular}{|c|l|}
\hline Attribute & \\
\hline AnnotatedImage & $\begin{array}{l}\text { One of the key components in the process of identification of } \\
\text { the images. The method AnnotatedImage includes functions } \\
\text { like NoFaceFound, MultipleFaceFound, LeftEyeNotFound, } \\
\text { RightEyeNotFound, BothEyesNotFound. }\end{array}$ \\
\hline FaceFinder & $\begin{array}{l}\text { As determined by the face, FaceFinder identifies the face } \\
\text { location based on the face center position, face width, face } \\
\text { rotation angle and confidence level. }\end{array}$ \\
\hline FIRFactory & $\begin{array}{l}\text { A tool which creates FIR (Face Identification Record) } \\
\text { instances and saves them to a file or byte array. }\end{array}$ \\
\hline Population & $\begin{array}{l}\text { This attribute is an ordered set of named FIR's which } \\
\text { represents the population used for identifications. }\end{array}$ \\
\hline FaceMatcher & $\begin{array}{l}\text { A tool for matching the query FIR against other FIR's in the } \\
\text { gallery. }\end{array}$ \\
\hline
\end{tabular}

\subsection{Matching Experiments}

While working with the NeuroTechnology face matcher, the matching is accomplished by comparison of templates. In comparison, when using Cognitec FaceVACs Technology, the facial 
information is read as a FIR (Facial Information Record) file in the enrollment process, which is further processed for identification to generate the similarity score. The greater the similarity score, the greater the chance that the matching of features from the probe image are from the same individual in the gallery set or the available dataset.

The image comparison can be assessed by the match score generated, which indicates the degree of similarity of a probe image compared with a gallery image in a dataset.

The following list of matching experiments are performed in this project.

1. Frontal (probe) to Frontal (gallery) images, to get a sense of the baseline match score distribution for ideal matching circumstances

2. Non-ideal pose-varying (probe) to frontal (gallery)

3. Pseudo-pose-corrected (probe) to frontal (gallery)

4. Auto-pose-corrected (probe) to frontal (gallery)

In order to generate the match scores, the images need to be enrolled by the system for each of the 4 matching experiments listed above. Due to the challenges imposed by non-frontal images, the matchers were not able to enroll images within the full range of pose angles generated using Mesh Lab The number of subjects enrolled for each angle of pitch, yaw, and coupled pitch/yaw rotations for each of the matchers are shown in Table 4.3, 4.4, and 4.5. In reviewing this data, it can be observed that, as the degree of rotation increases the rate of enrollment has decreased. The NeuroTechnology matcher has been able to detect faces up to $40^{\circ}$ and $-50^{\circ}$ pitch rotation, up to $30^{\circ}$ yaw rotation and only two angles of coupled pitch $30^{\circ}$ yaw $15^{\circ}$ and coupled pitch $-30^{\circ}$ yaw $15^{\circ}$. Cognitec FaceVACs has been able to detect faces up to $50^{\circ}$ and $-60^{\circ}$ pitch rotation, up to $50^{\circ}$ yaw rotation and Pitch $30^{\circ}$ Yaw $15^{\circ}$, Pitch $30^{\circ}$ Yaw $30^{\circ}$, Pitch $-30^{\circ}$ Yaw $-15^{\circ}$, Pitch $-30^{\circ}$ Yaw $30^{\circ}$. 


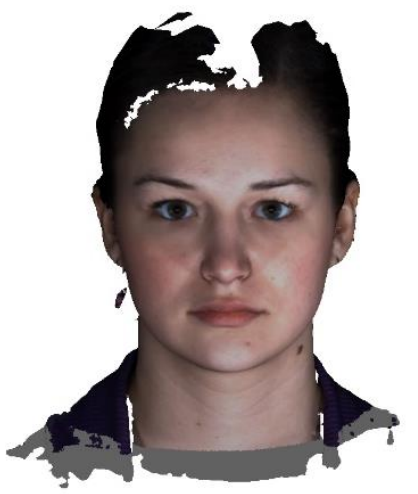

$10^{\circ}$ Pitch

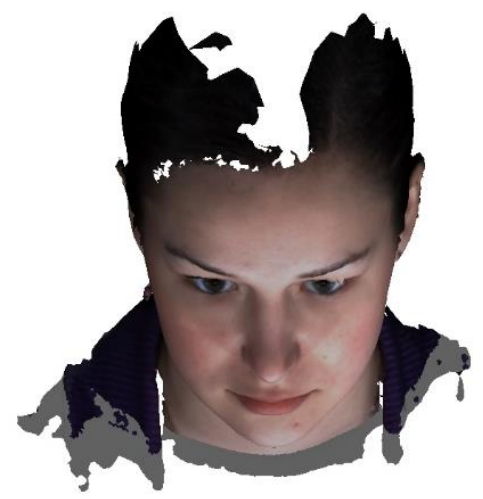

$40^{\circ}$ Pitch

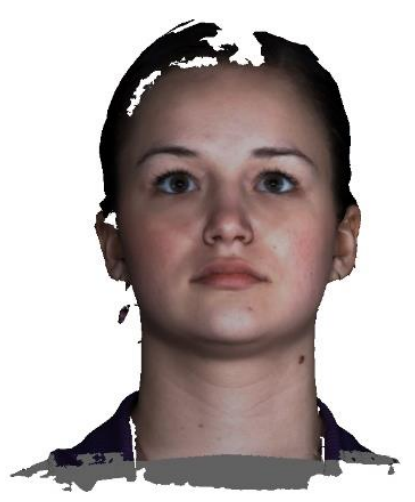

$-10^{\circ}$ Pitch

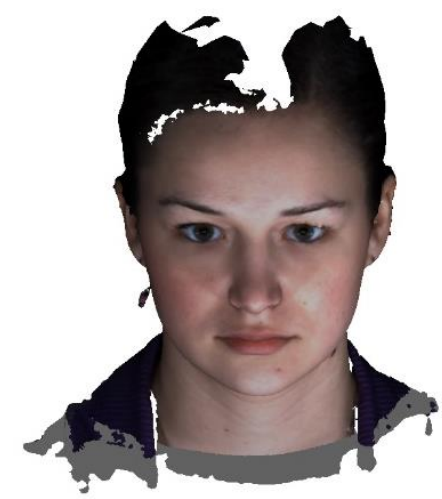

$20^{\circ}$ Pitch

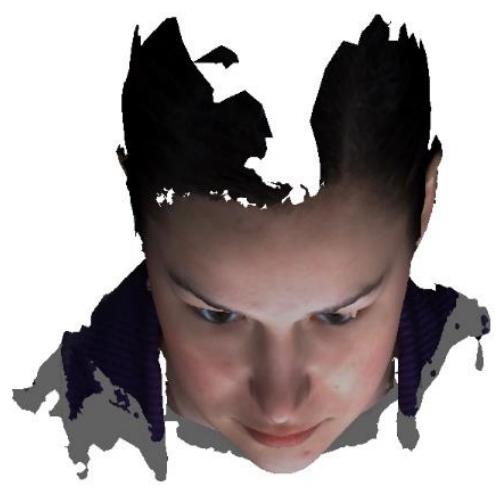

$50^{\circ}$ Pitch

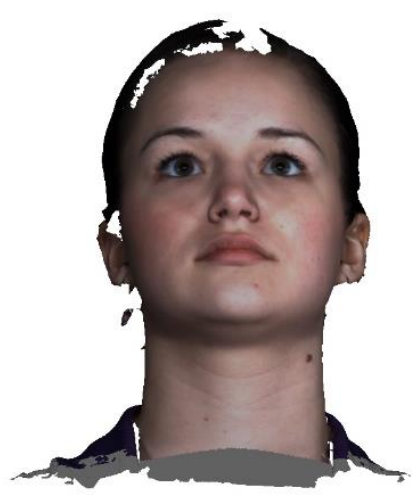

$-20^{\circ}$ Pitch

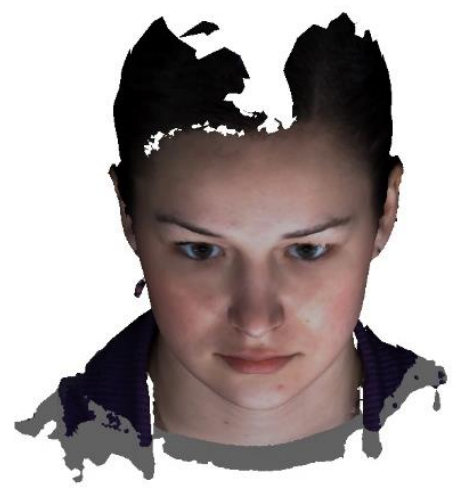

$30^{\circ}$ Pitch

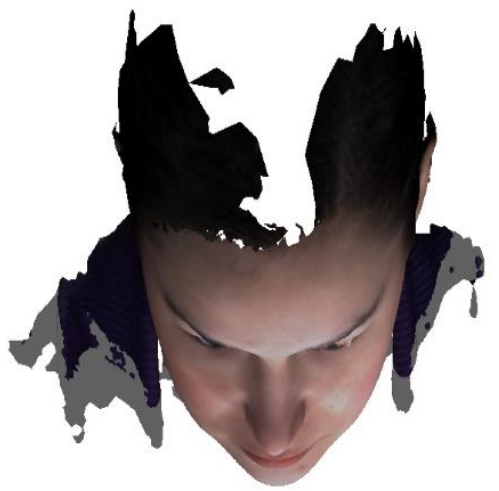

$60^{\circ}$ Pitch

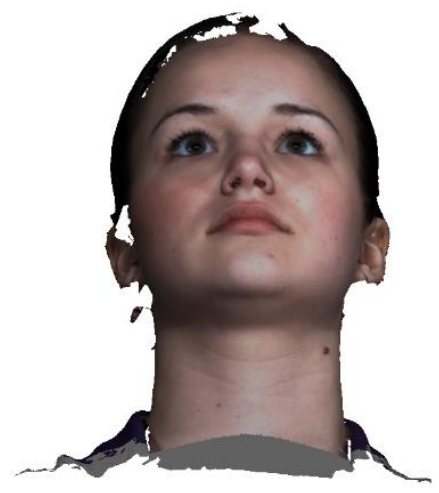

$-30^{\circ}$ Pitch 


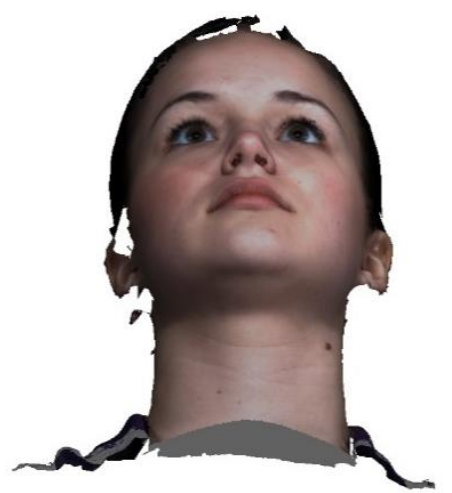

$-40^{\circ}$ Pitch

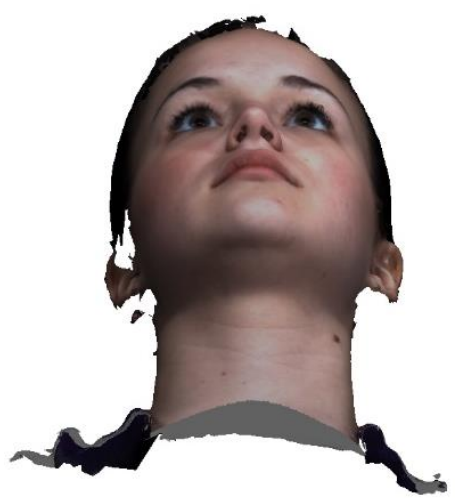

$-50^{\circ}$ Pitch

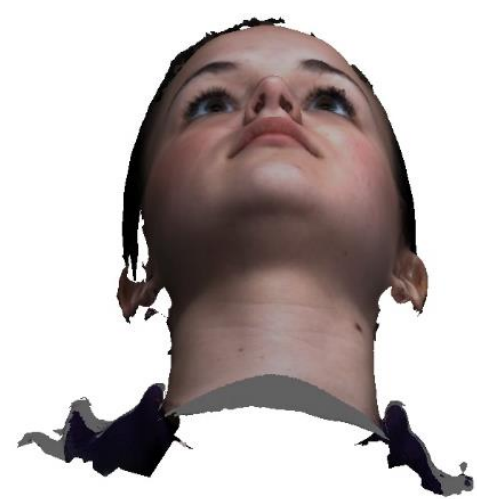

$-60^{\circ}$ Pitch

Figure 4.5 Pose Varying Pitch angles

The non-frontal images generated with pitch variations can be observed from Figure 4.5 from $-10^{\circ}$ to $-60^{\circ}$ and $10^{\circ}$ to $60^{\circ}$. It can also be observed from Table 4.3 that the enrollment of variation in angle is different for both SDK's. Cognitec FaceVACs can enroll non-frontal poses with up to $60^{\circ}$ and $60^{\circ}$ of pitch rotation, while NeuroTechnology failed to enroll images with rotations greater than $-50^{\circ}$ and $40^{\circ}$ of pitch.

Table 4.3 Number of images in each probe set for pitch rotation

\begin{tabular}{|c|c|c|}
\hline \multirow{4}{*}{ Matcher } & Image Group & $\begin{array}{c}\text { Number of Images } \\
\text { Enrolled }\end{array}$ \\
\hline \multirow{4}{*}{ Cognitec FaceVACs } & Pitch $10^{\circ}$ & 100 \\
\cline { 2 - 3 } SDK & Pitch $20^{\circ}$ & 96 \\
\cline { 2 - 3 } & Pitch $30^{\circ}$ & 96 \\
\cline { 2 - 3 } & Pitch $40^{\circ}$ & 85 \\
\cline { 2 - 3 } & Pitch $50^{\circ}$ & 38 \\
\cline { 2 - 3 } & Pitch $60^{\circ}$ & 4 \\
\cline { 2 - 3 } & Pitch $-10^{\circ}$ & 99 \\
\cline { 2 - 3 } & Pitch $-20^{\circ}$ & 99 \\
\cline { 2 - 3 } & Pitch $-30^{\circ}$ & 99 \\
\hline
\end{tabular}




\begin{tabular}{|c|c|c|}
\hline \multirow{4}{*}{} & Pitch $-50^{\circ}$ & 78 \\
\cline { 2 - 3 } & Pitch $-60^{\circ}$ & 27 \\
\hline \multirow{4}{*}{$\begin{array}{c}\text { NeuroTechnology } \\
\text { MegaMatcher }\end{array}$} & Pitch $10^{\circ}$ & 95 \\
\cline { 2 - 3 } & Pitch $20^{\circ}$ & 84 \\
\cline { 2 - 3 } & Pitch $30^{\circ}$ & 40 \\
\cline { 2 - 3 } & Pitch $40^{\circ}$ & 7 \\
\cline { 2 - 3 } & Pitch $-10^{\circ}$ & 95 \\
\cline { 2 - 3 } & Pitch $-20^{\circ}$ & 70 \\
\cline { 2 - 3 } & Pitch $-30^{\circ}$ & 30 \\
\cline { 2 - 3 } & Pitch $-40^{\circ}$ & 7 \\
\hline
\end{tabular}

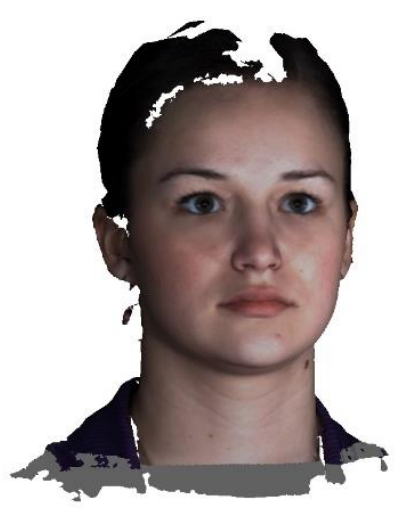

$10^{\circ}$ Yaw

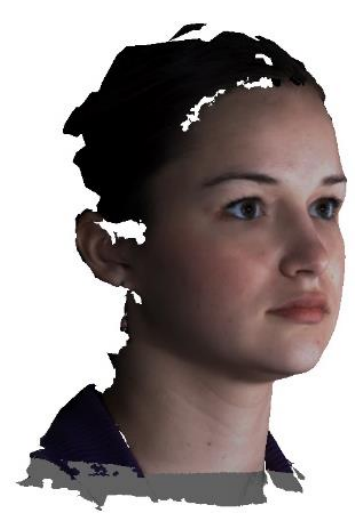

$40^{\circ} \mathrm{Yaw}$

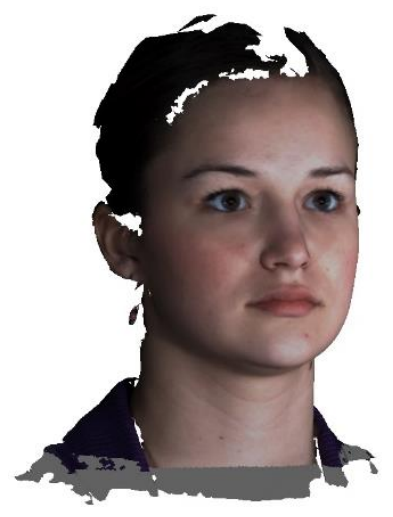

$20^{\circ}$ Yaw

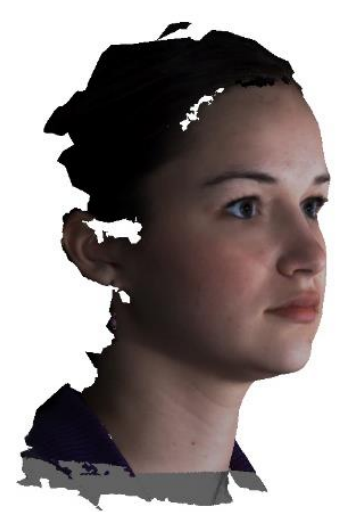

$50^{\circ}$ Yaw

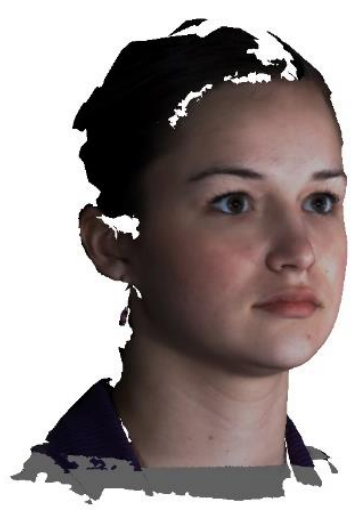

$30^{\circ} \mathrm{Yaw}$

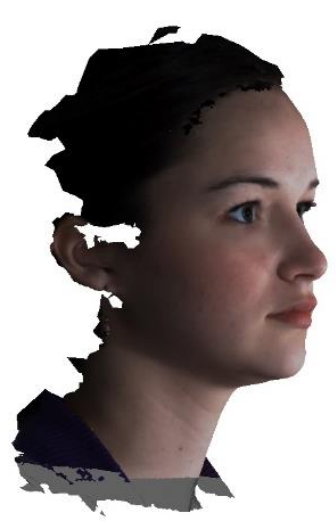

$60^{\circ}$ Yaw 
Figure 4.6 Pose Varying Yaw Angles

The non-frontal images with yaw angle variations are generated for 6 different angles from $10^{\circ}$ to $60^{\circ}$ can be seen in Figure 4.6. The number of images enrolled for each angle in both matchers is as shown in Table 4.4. For yaw variations, NeuroTechnology matcher could enroll only up to $30^{\circ}$ angle rotation whereas Cognitec FaceVACs could detect up to $50^{\circ}$ angle rotation which means Cognitec matcher has a better rate of enrollment of images with greater angle of rotation.

Table 4.4 Number of images in each probe set for yaw rotation

\begin{tabular}{|c|c|c|}
\hline Matcher & Image Group & $\begin{array}{c}\text { Number of Images } \\
\text { Enrolled }\end{array}$ \\
\hline \multirow{5}{*}{$\begin{array}{c}\text { Cognitec FaceVACs } \\
\text { SDK }\end{array}$} & Yaw $10^{\circ}$ & 100 \\
\hline & Yaw $20^{\circ}$ & 100 \\
\hline & Yaw $30^{\circ}$ & 98 \\
\hline & Yaw $40^{\circ}$ & 68 \\
\hline & Yaw $50^{\circ}$ & 10 \\
\hline \multirow{3}{*}{$\begin{array}{c}\text { NeuroTechnology } \\
\text { MegaMatcher }\end{array}$} & Yaw $10^{\circ}$ & 87 \\
\hline & Yaw $20^{\circ}$ & 32 \\
\hline & Yaw $30^{\circ}$ & 5 \\
\hline
\end{tabular}

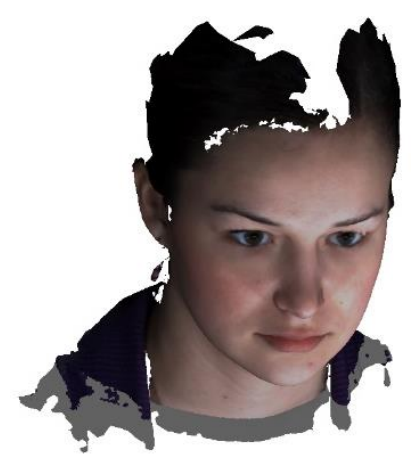

Pitch $30^{\circ}$ Yaw $15^{\circ}$

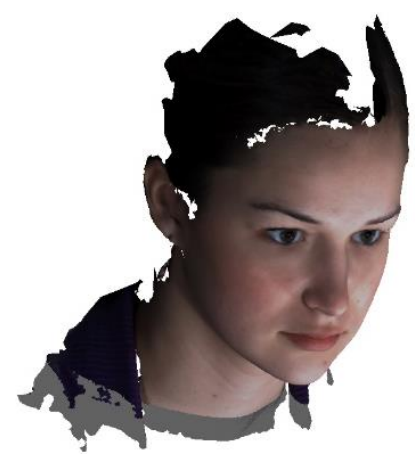

Pitch $30^{\circ}$ Yaw $30^{\circ}$

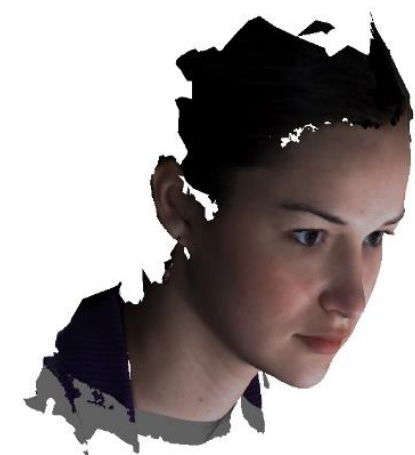

Pitch $30^{\circ}$ Yaw $45^{\circ}$

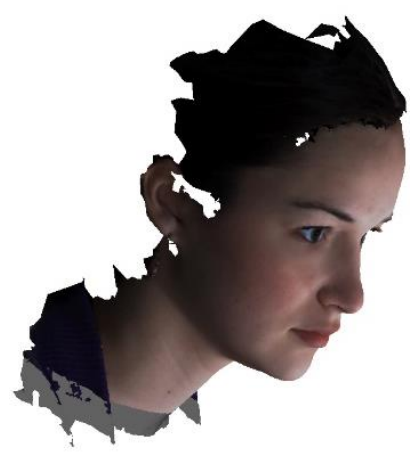

Pitch $30^{\circ}$ Yaw $60^{\circ}$ 


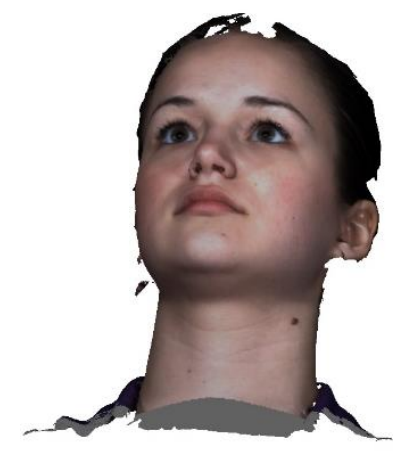

Pitch $-30^{\circ}$ Yaw $-15^{\circ}$

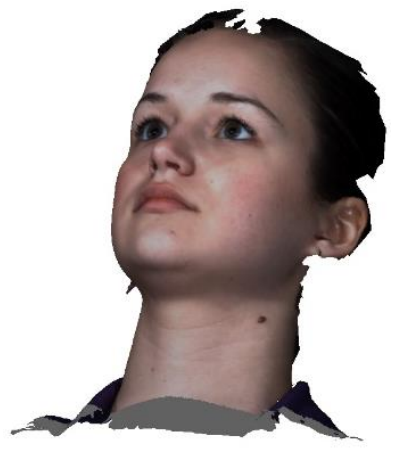

Pitch $-30^{\circ}$ Yaw $-30^{\circ}$

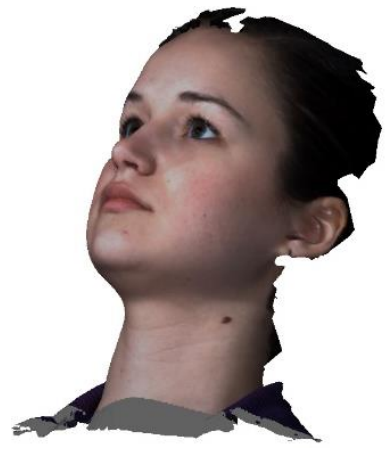

Pitch $-30^{\circ}$ Yaw $-45^{\circ}$

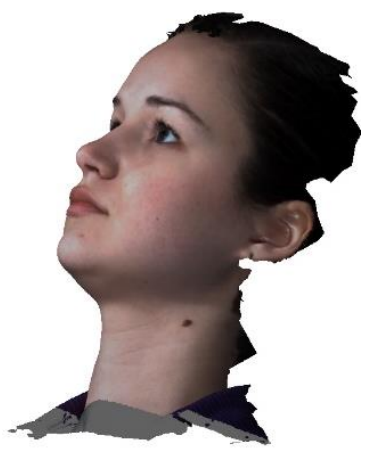

Pitch $-30^{\circ}$ Yaw $-60^{\circ}$

Figure 4.7 Pose Varying Coupled Pitch and Yaw Angles

In this experiment, both pitch and yaw angles were given to the $3 \mathrm{D}$ facial images, as shown in Figure 4.7 and are generated in 8 different combinations. The non-frontal coupled pitch and yaw images which are detected and enrolled by the two matchers are as shown in Table 4.5, where Cognitec can detect and enroll 6 combinations of pitch and yaw out of 8 combinations whereas NeuroTechnology could detect and enroll only 3 angular rotations.

Table 4.5 Number of images in each probe set for coupled (pitch and yaw) rotation

\begin{tabular}{|c|c|c|}
\hline \multirow{4}{*}{ Matcher } & Image Group & $\begin{array}{c}\text { Number of Images } \\
\text { Enrolled }\end{array}$ \\
\hline \multirow{4}{*}{$\begin{array}{c}\text { Cognitec FaceVACs } \\
\text { SDK }\end{array}$} & Coupled $30^{\circ} 15^{\circ}$ & 96 \\
\cline { 2 - 3 } & Coupled $30^{\circ} 30^{\circ}$ & 74 \\
\cline { 2 - 3 } & Coupled $30^{\circ} 45^{\circ}$ & 9 \\
\cline { 2 - 3 } & Coupled $-30^{\circ}-15^{\circ}$ & 98 \\
\cline { 2 - 3 } & Coupled $-30^{\circ}-30^{\circ}$ & 91 \\
\hline \multirow{2}{*}{$\begin{array}{c}\text { NeuroTechnology } \\
\text { MegaMatcher }\end{array}$} & Coupled $-30^{\circ}-45^{\circ}$ & 32 \\
\cline { 2 - 3 } & Coupled $30^{\circ} 15^{\circ}$ & 21 \\
\cline { 2 - 3 } & Coupled $-30^{\circ}-15^{\circ}$ & 50 \\
\hline
\end{tabular}




\subsection{Performance of Baseline Experiment}

The baseline experiment is conducted for frontal (probe) to frontal (gallery) to get the match score distribution for ideal matching circumstances. The baseline performance is tested on both Cognitec FaceVACs and NeuroTechnology matchers. The rank accuracy is $100 \%$ in both the matchers and is presented in the CMC curves as shown in Figure 4.8 (a), (b). The reason for attaining a 100\% rank accuracy is due to the controlled nature of the data collection and the ideal value will provide a good baseline to evaluate the match performance for increasing non-ideality (in this case, it is non-frontal pose angle).

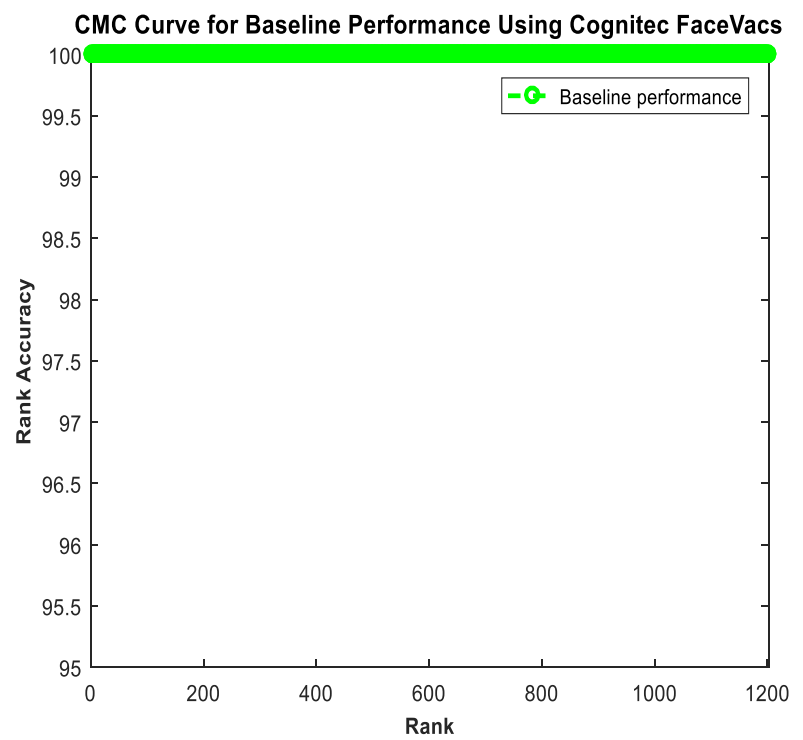

(a)

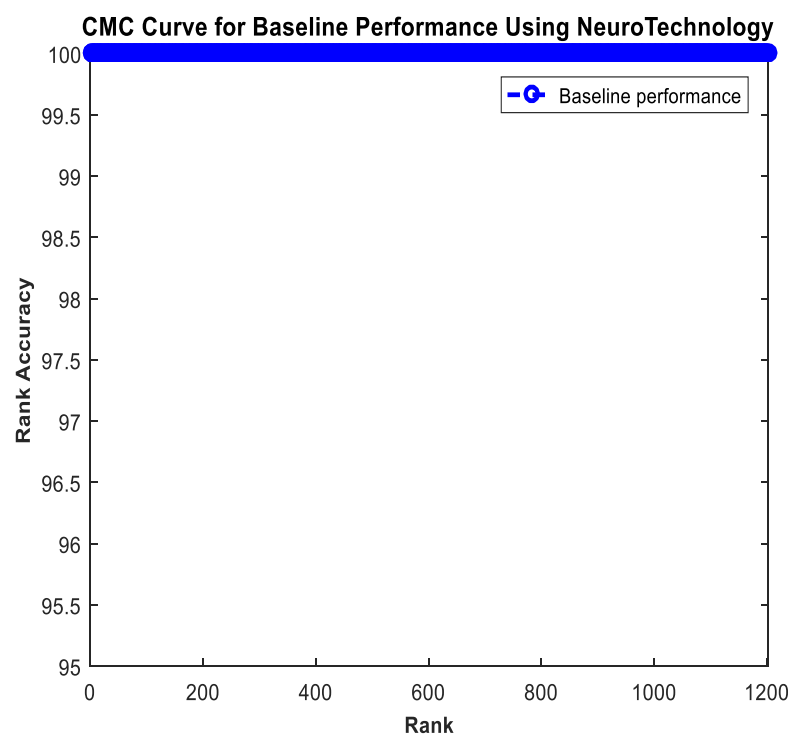

(b)

Figure 4.8 CMC Curve representation for baseline experiments, (a) CMC Curve for baseline experiment using Cognitec FaceVACs, (b) CMC Curve for baseline experiment using NeuroTechnology

\subsection{Performance Evaluation for Pose Varying Images}

As discussed earlier in this report, identification tests are performed in this project to assess the match performance of pose-corrected non-frontal face images using commercial matchers. CMC (Cumulative Match Characteristics) are one of the most widely used metrics considered to evaluate 
the results of facial matching experiment. The CMC's generated for different probe sets against a fixed gallery as listed in section 4.4 set are discussed in the following sections.

\subsubsection{Uncorrected Pitch Variations}

The performance of the pose varying images was evaluated using both the NeuroTechnology and Cognitec FaceVACs matchers. The rank accuracies of varying angle rotations are shown in the CMC curves in Figure 4.9 (a), (b).

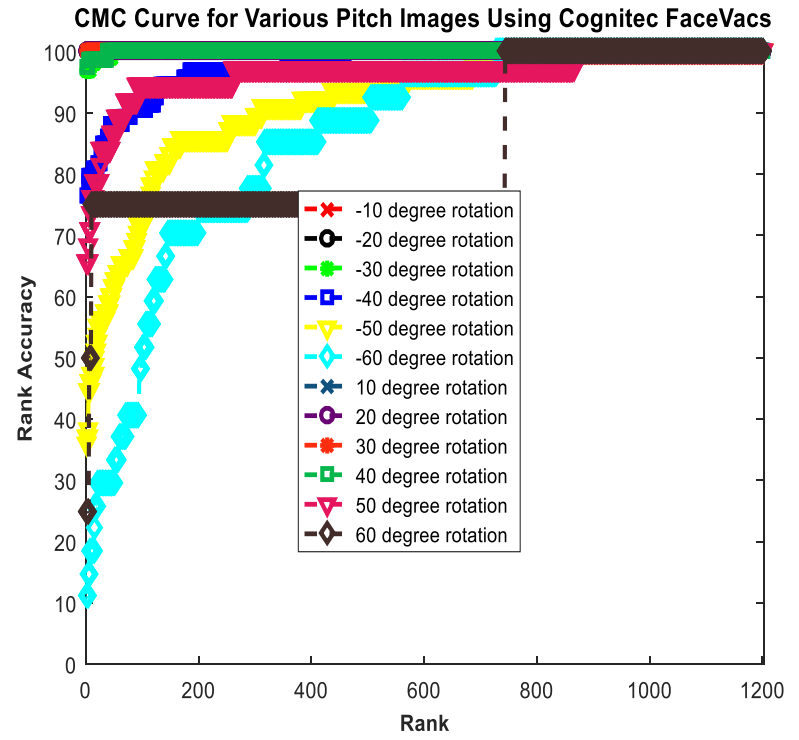

(a)

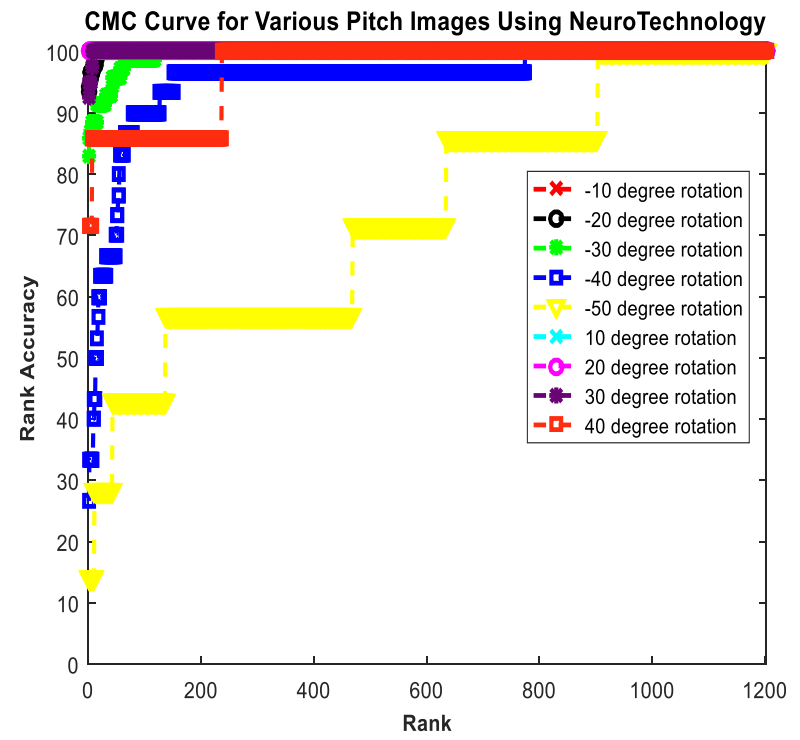

(b)

Figure 4.9 CMC curve representation of Uncorrected Pitch Variations Using Cognitec and NeuroTechnology Matchers, (a) CMC for Uncorrected Pitch Variations Using Cognitec FaceVACs, (b) CMC for Uncorrected Pitch Variations Using NeuroTechnology

Observing the results, as the angle of rotation increases, the rank-1 accuracy decreased for both matchers. 


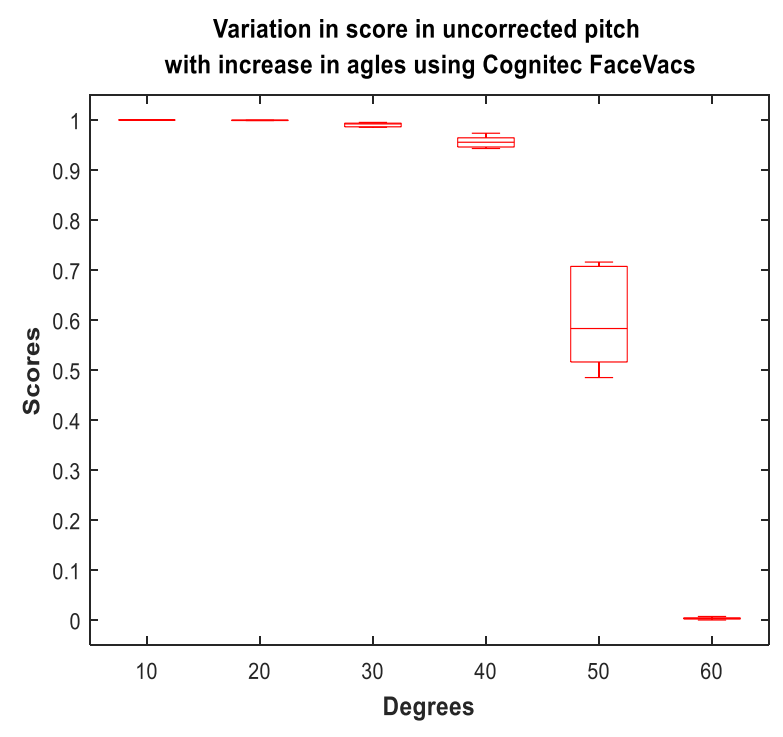

(a)

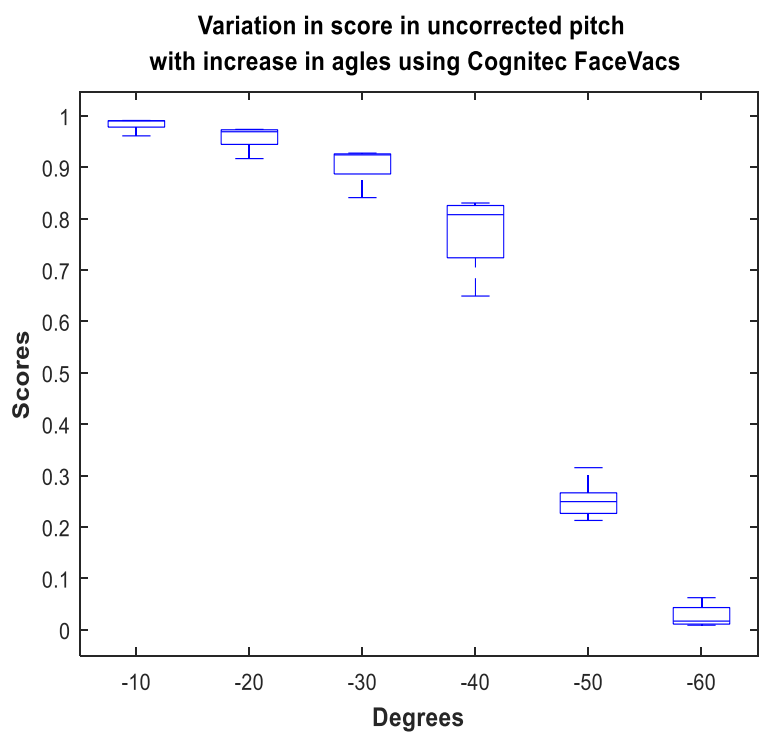

(b)

Figure 4.10 Match Score Distribution of Uncorrected Pitch Variations, (a) Variation in match score for pitch angles from $10^{\circ}$ to $60^{\circ}$ using Cognitec FaceVACs, (b) Variation in match score for pitch angles from $-10^{\circ}$ to $-60^{\circ}$ using Cognitec FaceVACs

The variation in match scores for pitch rotations with increasing angle is represented using box plots, as shown in Figure 4.10 (a), (b). As the angle of rotation increases there is noticible decrease in match score in both 4.10 (a) and 4.10 (b) where the match score dropped from around 0.99 to 0.001 for $10^{\circ}$ to $60^{\circ}$ similarly for $-10^{\circ}$ to $-60^{\circ}$. As discussed earlier, the size of these datasets are different because the number of images enrolled decreased with increase in angle of rotation for both matchers.

\subsubsection{Uncorrected Yaw Variations}

The performance evaluation of varying yaw rotations is shown in the CMC curves in Figure 4.11. The rank accuracy of yaw rotations for which enrollment was completed in the Cognitec FaceVACs software is $100 \%$, while the NeuroTechnology tool presented a decrease in rank accuracy for increase in yaw angle. The most likely reason for the high accuracy of the Cognitec tool is the small size of the data set used for testing and the high quality of images (which were obtained under controlled conditions with uniform background and lighting). Although there is 
$100 \%$ rank accuracy for the non-frontal yaw images there a decrease in match scores with increase in angle of rotation.

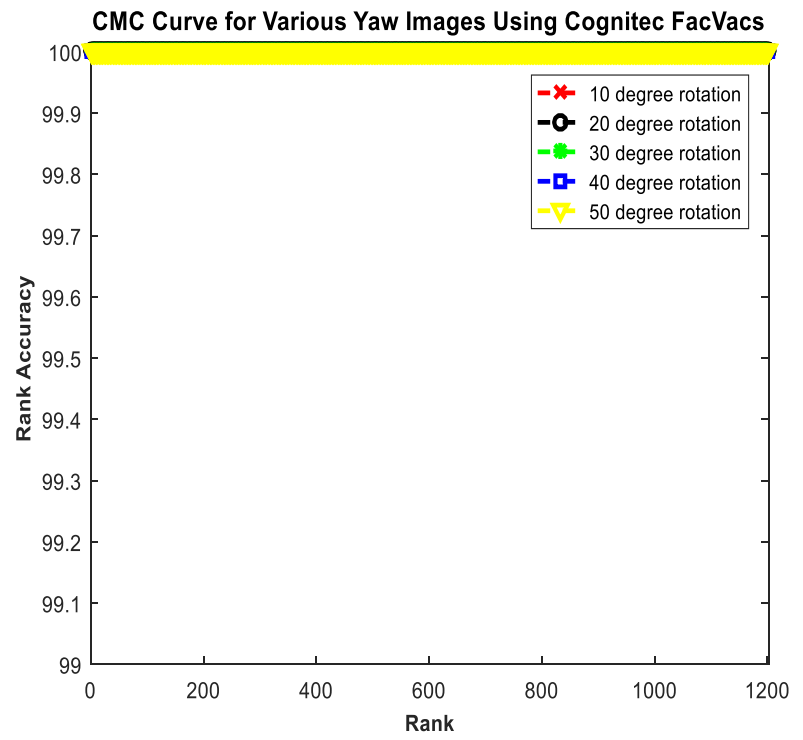

(a)

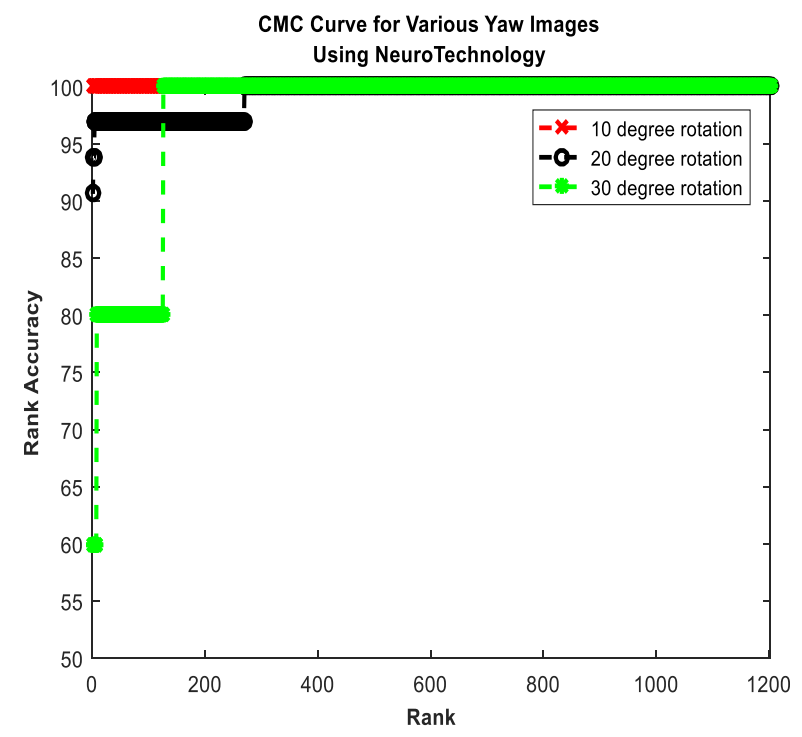

(b)

Figure 4.11 CMC curve representation of Uncorrected Yaw Variations Using Cognitec and NeuroTechnology Matchers, (a) CMC for Uncorrected Yaw Variations Using Cognitec FaceVACs, (b) CMC for Uncorrected Yaw Variations Using NeuroTechnology

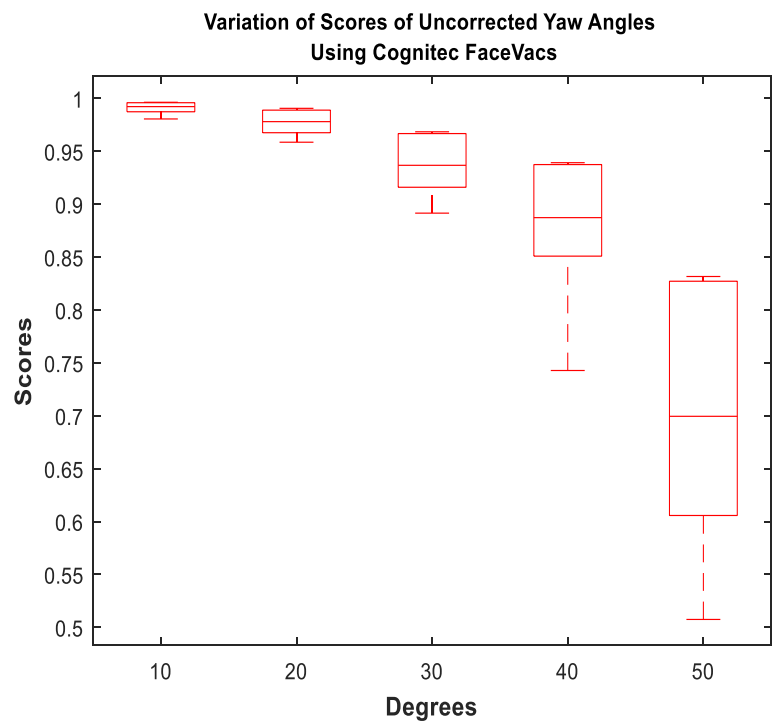

Figure 4.12 Match Score Distribution of Uncorrected Yaw Variation Using Cognitec FaceVACs 
The box plot in Figure 4.12 illustrates clearly the reduction in match score with the increase in angle of rotation for the Cognitec FaceVACs. It can be observed from the boxplot that there is a progressive drop in match score with the increase in angle of rotation from around 0.99 to 0.13 . Whereas NeuroTechnology could enroll only 3 angular variations in each case and the corresponding rank accuracy can be seen from Figure 4.11 (b) in which the rank-1 accuracy decreased with increase in angle of rotation.

\subsubsection{Uncorrected Coupled (Pitch and Yaw) Variations}

The variations in the rate of recognition of pitch and yaw angles is shown in the CMC curves in Figure 4.13. The rank accuracies of coupled pitch and yaw angles have decreased with the increase in angle of rotation. This result was consistent for both matchers tested. The distribution of match scores for different rotations can be observed from the box plots in Figure 4.14, showing a gradual decrease in the scores for increasing angle for both matchers.

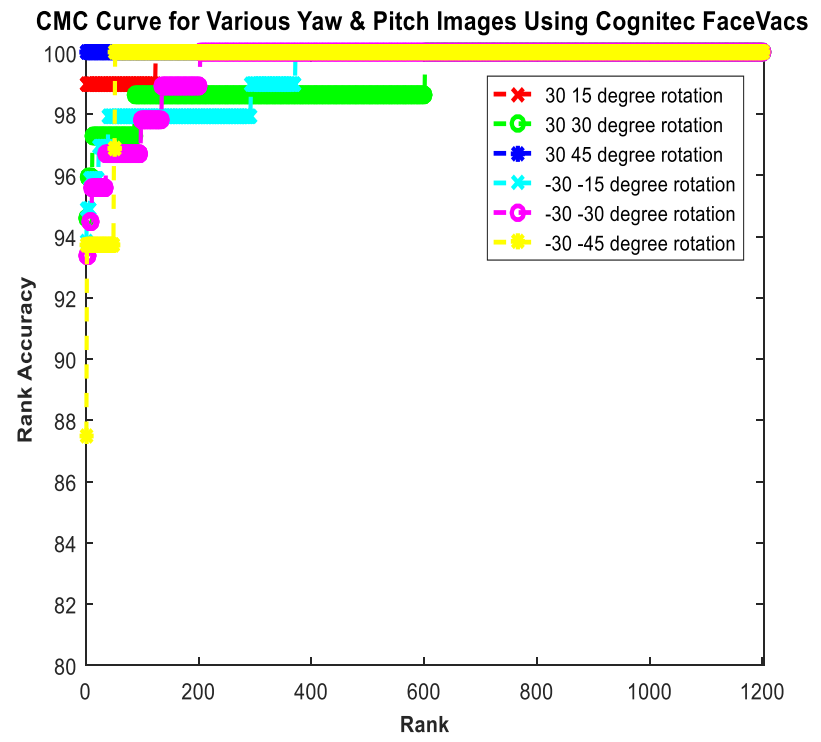

(a)

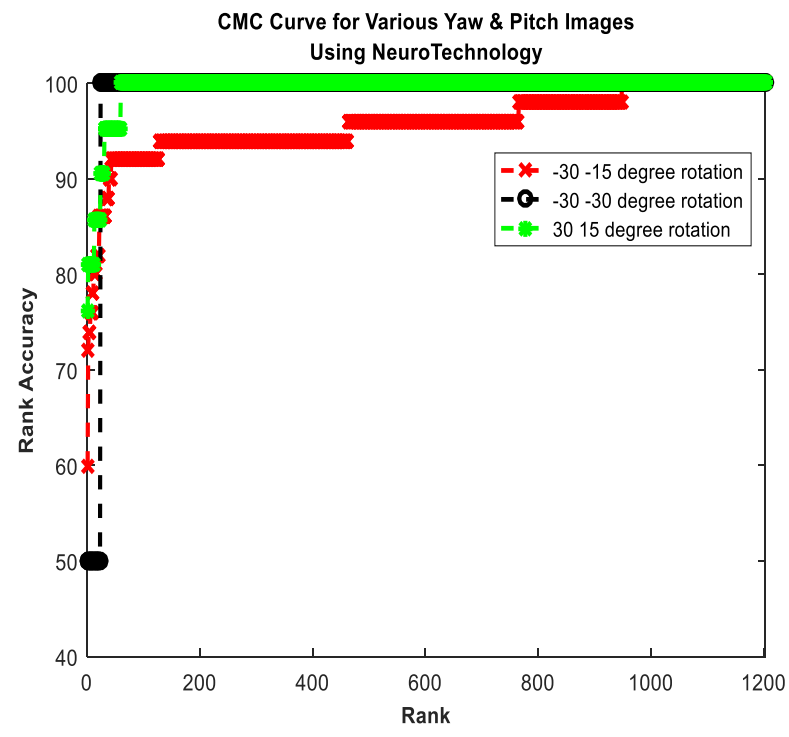

(b)

Figure 4.13 CMC curve representation of Uncorrected Pitch and Yaw Variations Using Cognitec and NeuroTechnology Matchers, (a) CMC for Uncorrected Pitch and Yaw Variations Using Cognitec FaceVACs, (b) CMC for Uncorrected Pitch and Yaw Variations Using NeuroTechnology 
The match score is varied from 0.92 to 0.5 for coupled pitch and yaw variations as shown in Figure 4.14 (a) and from 0.81 to 0.13 for coupled pitch and yaw variations in Figure 4.14 (b). Thus, with increase in pitch and yaw angles the match score has gradually decreased for each angle.

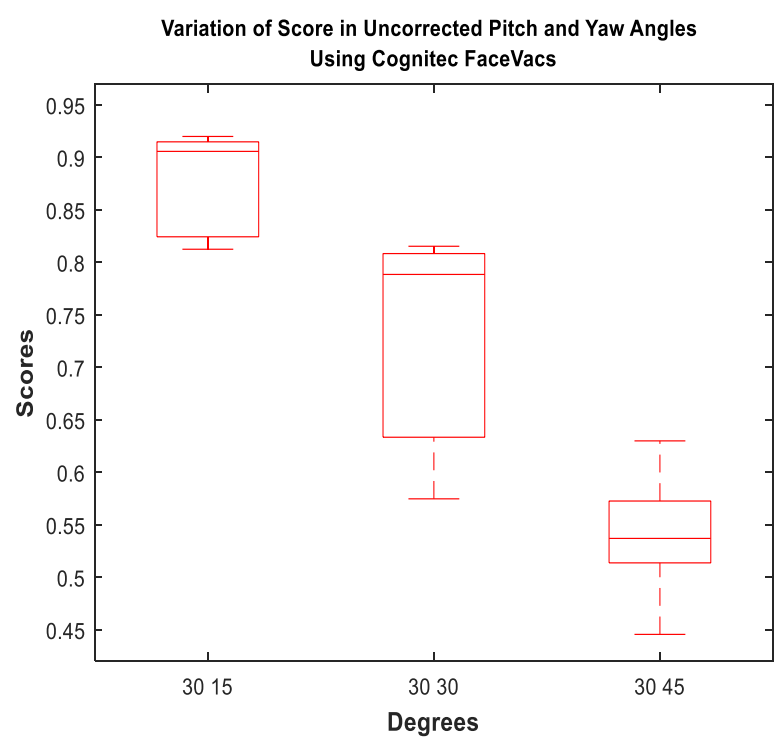

(a)

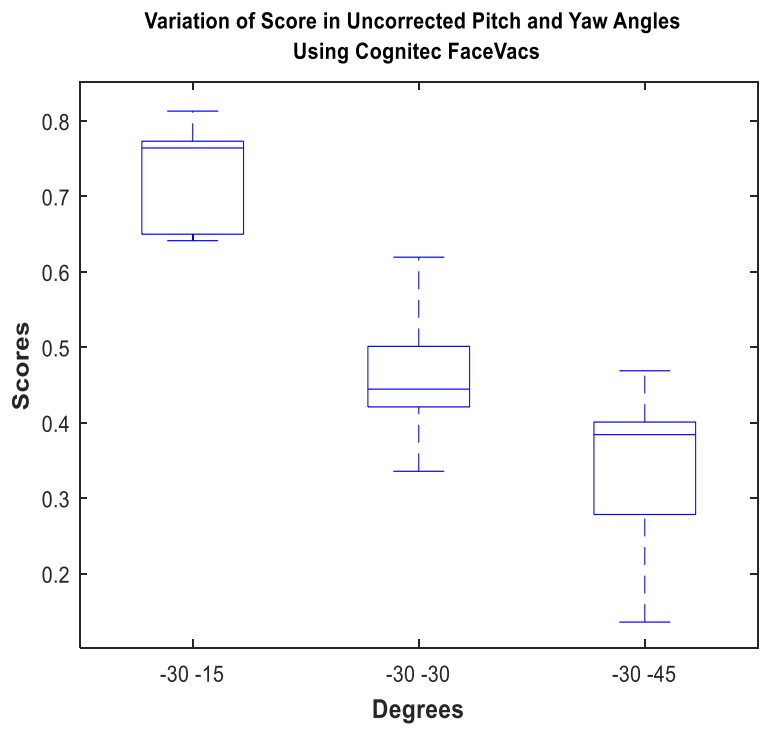

(b)

Figure 4.14 Match Score Distribution of Uncorrected Coupled Pitch and Yaw Variations Using Cognitec FaceVACs, (a) Match Score Variation of Enrolled Positive Coupled Pitch and Yaw variations, (b) Match Score Variation of Enrolled Negative Coupled Pitch and Yaw variations

\subsection{Performance Evaluation for Pseudo Pose Corrected Images}

\subsubsection{Performance of Pseudo Pose Corrected Pitch Variations Images}

The pose varying images are pseudo-pose-corrected by varying the polygon count in the occluded regions using the process outlined in Chapter 3. Three levels of polygon reduction were applied: $25 \%, 50 \%$ and $75 \%$. As with the non-corrected images, the match performance of pseudo pose corrected images was assessed using Cognitec and NeuroTechnology, with results represented using CMC curves in Figures 4.16, 4.17 and 4.18. 


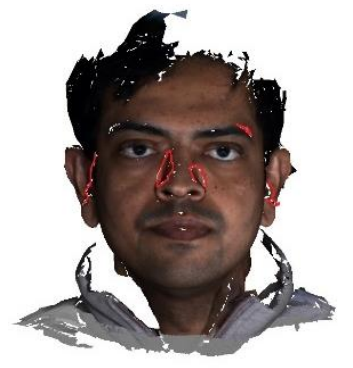

Pseudo Pose Corrected -

$40^{\circ}$ Pitch at $75 \%$ Polygon

Reduction

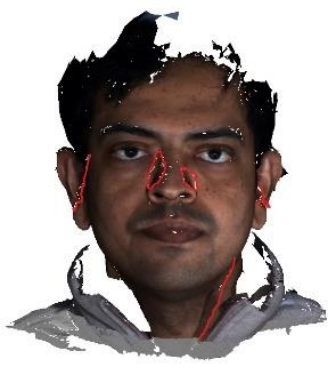

Pseudo Pose Corrected -

$40^{\circ}$ Pitch at $50 \%$ Polygon

Reduction

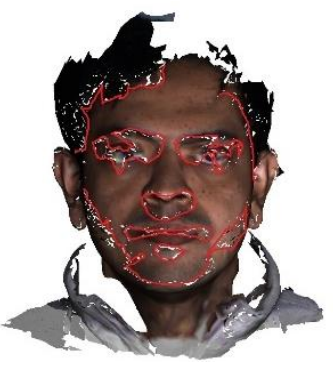

Pseudo Pose Corrected

$60^{\circ}$ Pitch at $75 \%$ Polygon

Reduction

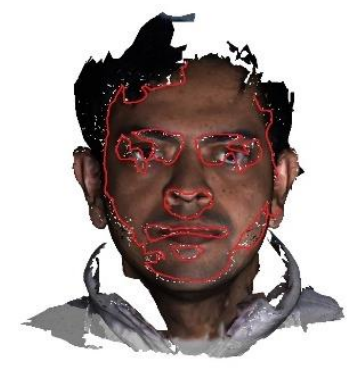

Pseudo Pose Corrected $60^{\circ}$ Pitch at $50 \%$ Polygon Reduction

Figure 4.15 Pseudo Pose Corrected Pitch at Varying Polygon Reduction

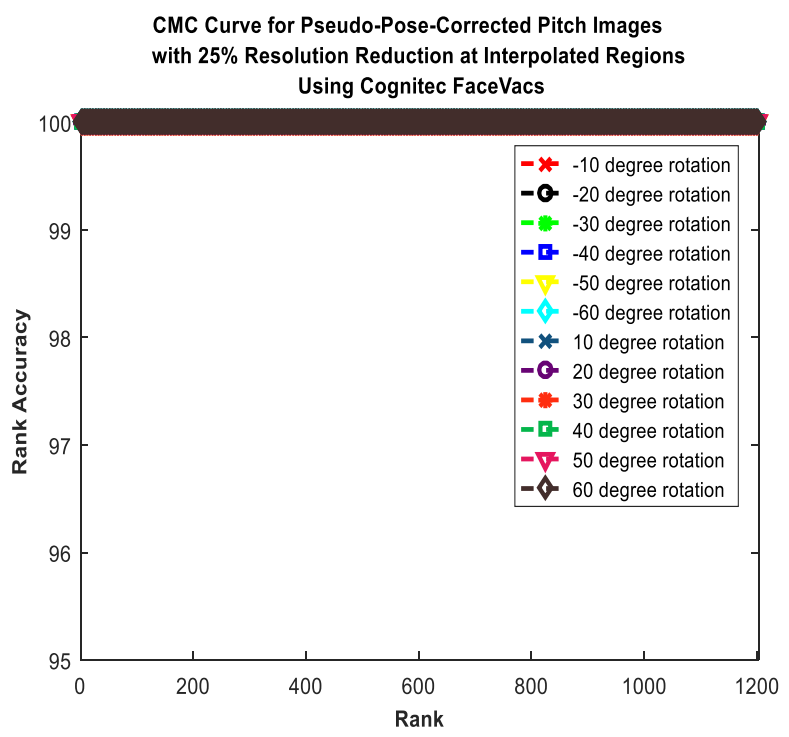

(a)

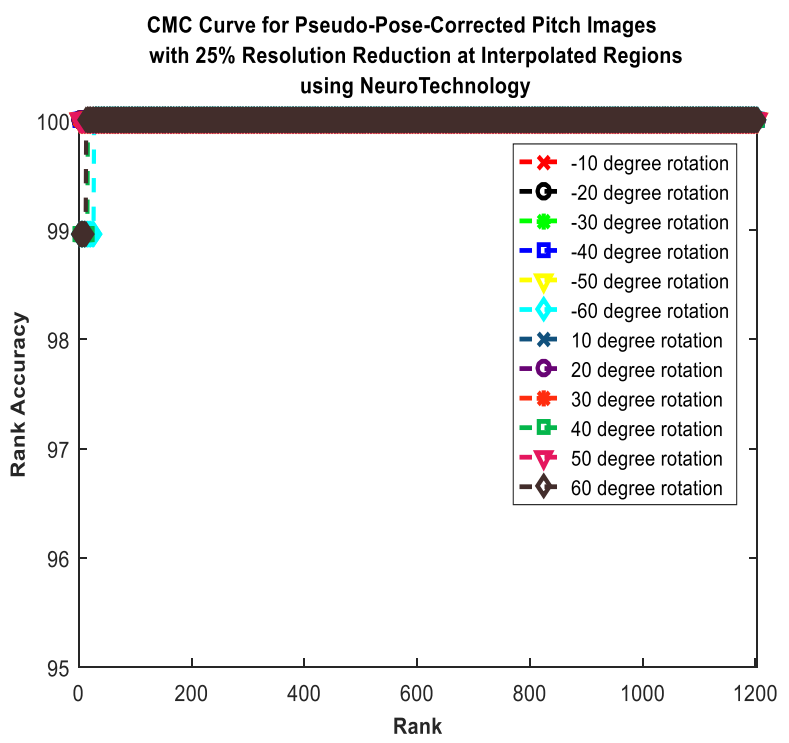

(b)

Figure 4.16 CMC of Pseudo Pose Corrected Pitch at 25\% Polygon Reduction Using Cognitec and NeuroTechnology Matchers, (a) CMC of Pseudo Pose Corrected Pitch at 25\% Polygon Reduction Using Cognitec FaceVACs, (b) CMC of Pseudo Pose Corrected Pitch at 25\% Polygon Reduction Using NeuroTechnology 


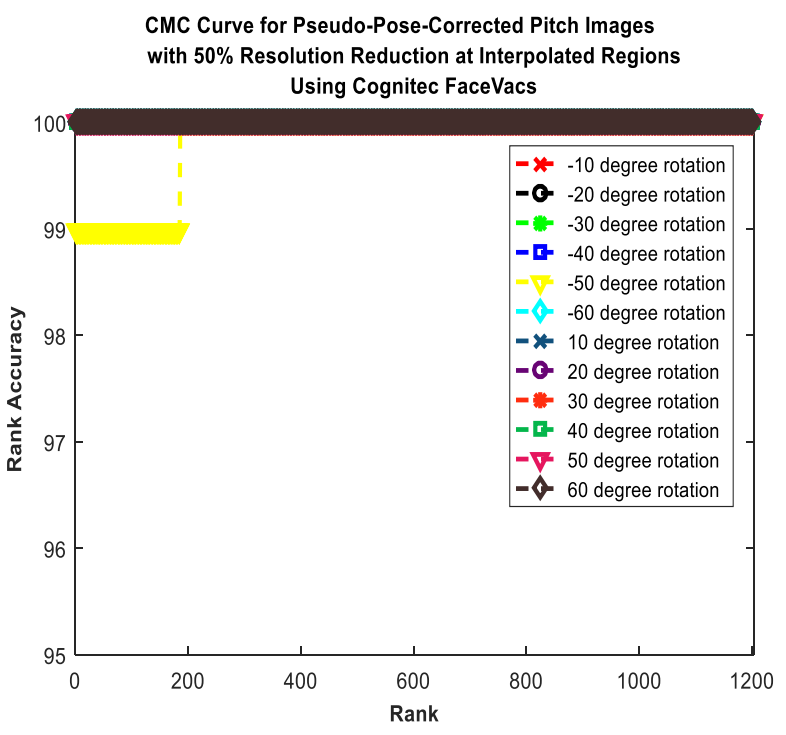

(a)

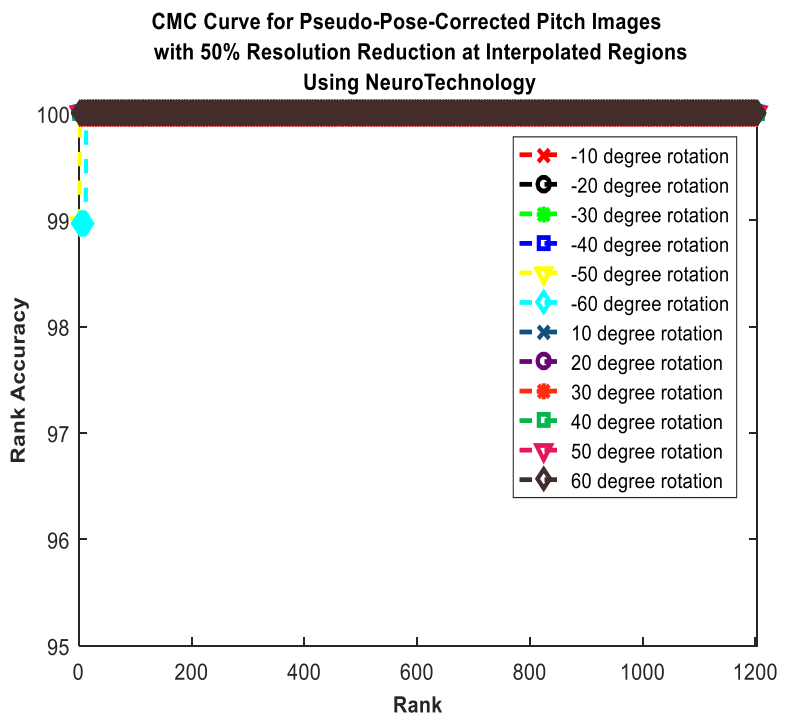

(b)

Figure 4.17 CMC of Pseudo Pose Corrected Pitch at 50\% Polygon Reduction Using Cognitec and NeuroTechnology Matchers, (a) CMC of Pseudo Pose Corrected Pitch at 50\% Polygon Reduction Using Cognitec FaceVACs, (b) CMC of Pseudo Pose Corrected Pitch at 50\% Polygon Reduction Using NeuroTechnology

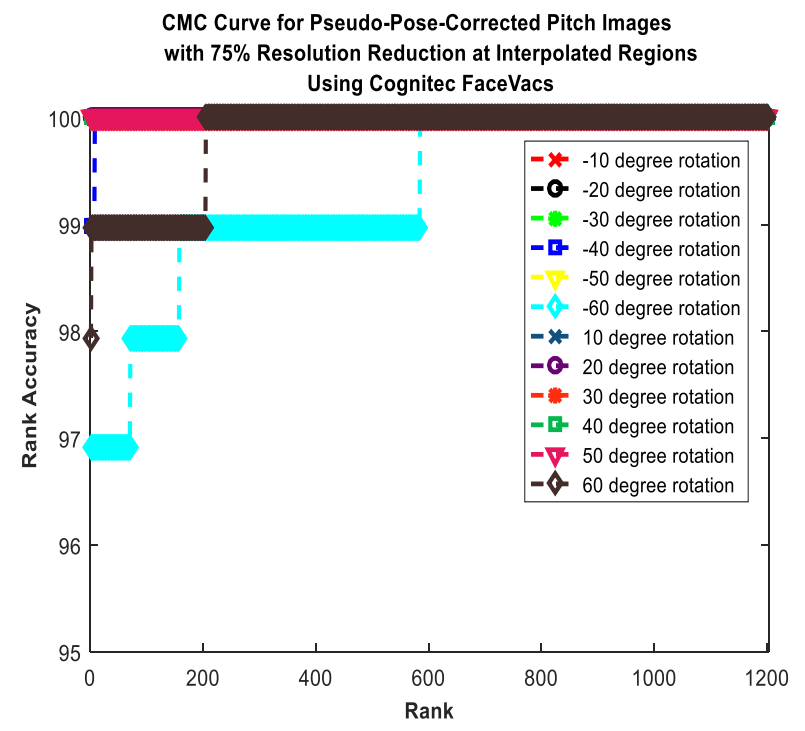

(a)

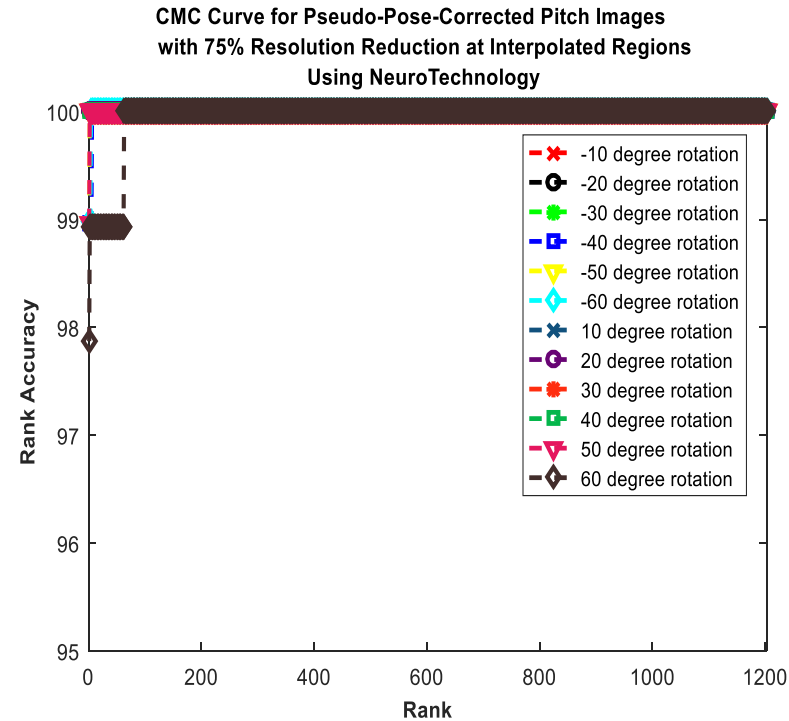

(b) 
Figure 4.18 CMC of Pseudo Pose Corrected Pitch at 75\% Polygon Reduction Using Cognitec and NeuroTechnology Matchers, (a) CMC of Pseudo Pose Corrected Pitch at 75\% Polygon Reduction Using Cognitec FaceVACs, (b) CMC of Pseudo Pose Corrected Pitch at 75\% Polygon Reduction Using NeuroTechnology

As can be observed from the curves for the pseudo-pose-corrected images, those with $75 \%$ polygon reduction there is much decrease in rank-1 accuracy compared to $50 \%$ and $25 \%$ polygon reduced images. When compared to the non-frontal and non-pose-corrected pitch images the performance of pseudo-pose corrected images has improved. As discussed earlier both Cognitec and NeuroTechnology matchers were not able to detect and enroll all the angular pitch rotations whereas in pseudo-pose-corrected images all the pitch angles with higher degree of rotation and maximum polygon reduction were also detected and enrolled. But there is variation in match score with increased polygon reduction as shown in the box plots below.

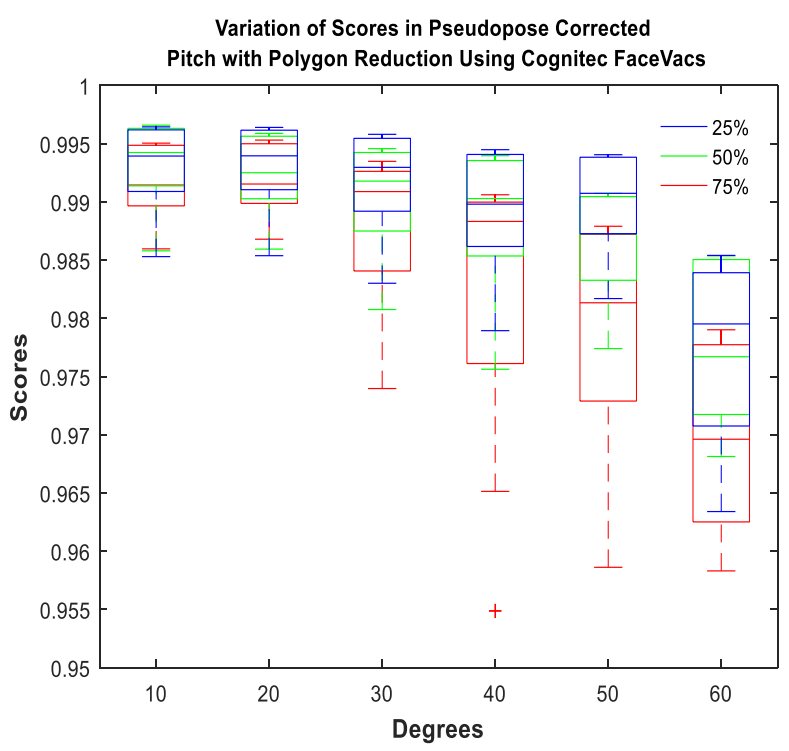

(a)

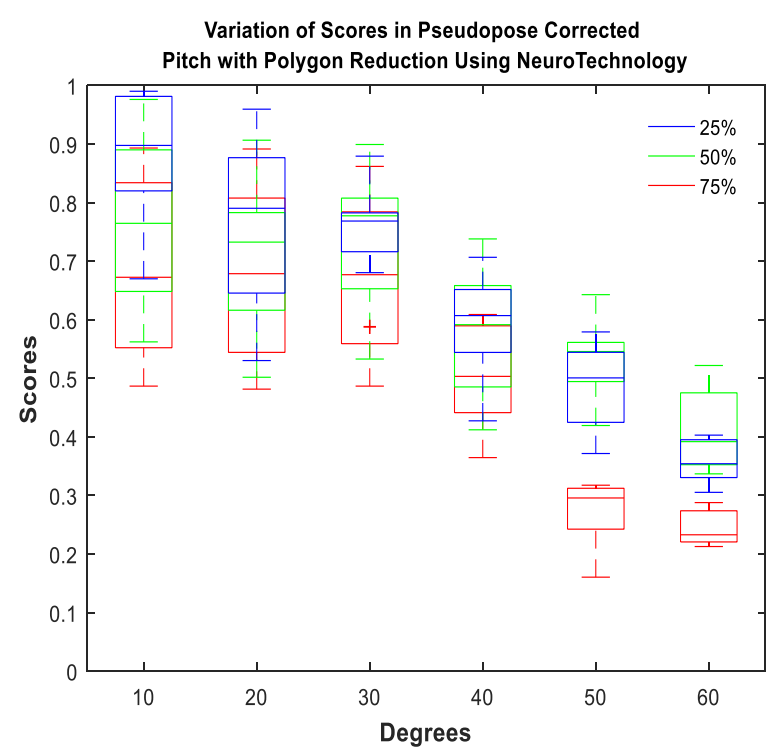

(b)

Figure 4.19 Match Score Distribution of Pseudo Pose Corrected Pitch with Varying Polygon Count, (a) Match Score Variations in Pseudo Pose Corrected Pitch Using Cognitec FaceVACs, (b) Match Score Variations in Pseudo Pose Corrected Pitch Using NeuroTechnology 
The box plost shown in Figure 4.19 (a), (b) shows the variation in match score for pseudo-posecorrection images with varying polygon count for both Cognitec and NeuroTechnology matchers. The match score distribution of $25 \%, 50 \%$ and $75 \%$ polygon reduction in the box plots is indicated in blue, green and red respectively. This data shows that an increase in angle and reduction of polygon count causes the match score to decrease, even for pose-corrected images. Thus even pseudo-pose corrected images performed better over non-pose corrected images, there is drop in performance with increase in angle and polygon reduction.

\subsubsection{Performance of Pseudo Pose Corrected Yaw Variations Images}

The pose-varying yaw images are pseudo-pose-corrected in a similar manner as pitch images with three levels of polygon reduction: $25 \%, 50 \%$ and $75 \%$. Performance of pseudo-pose-corrected yaw images with different polygon reductions at the interpolated regions is illustrated in the CMC curves shown in Figure 4.21, 4.22 and 4.23. As seen from the plots there is almost 100\% accuracy for every angle in both matchers. This can occur due to the small size of the data considered in the experiment and the images used were acquired under controlled conditions with uniform illumination and background.

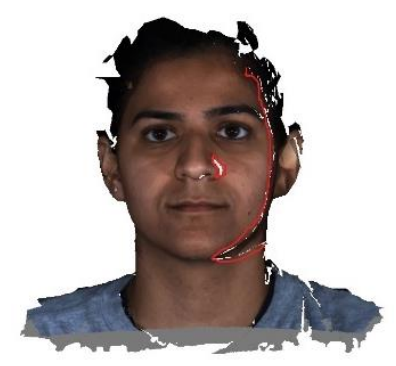

Pseudo Pose Corrected $20^{\circ}$ Yaw at $75 \%$ Polygon Reduction

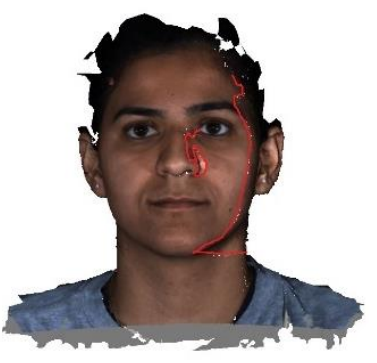

Pseudo Pose Corrected $30^{\circ}$ Yaw at $25 \%$ Polygon Reduction

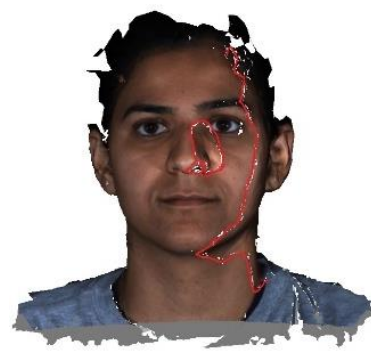

Pseudo Pose Corrected $40^{\circ}$ Yaw at $50 \%$ Polygon Reduction

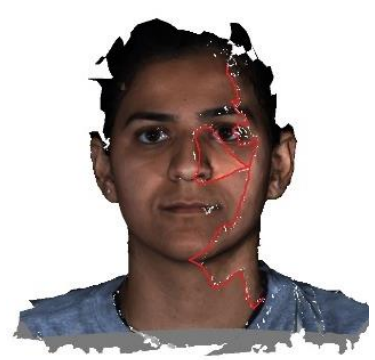

Pseudo Pose Corrected $50^{\circ}$ Yaw at $50 \%$ Polygon Reduction

Figure 4.20 Pseudo Pose Corrected Yaw at Varying Polygon Reduction 


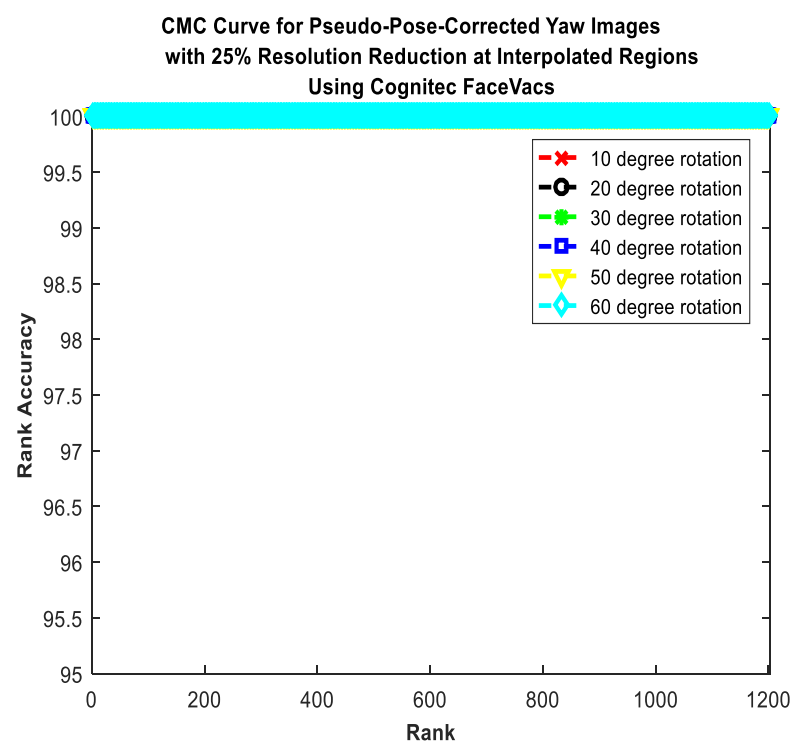

(a)

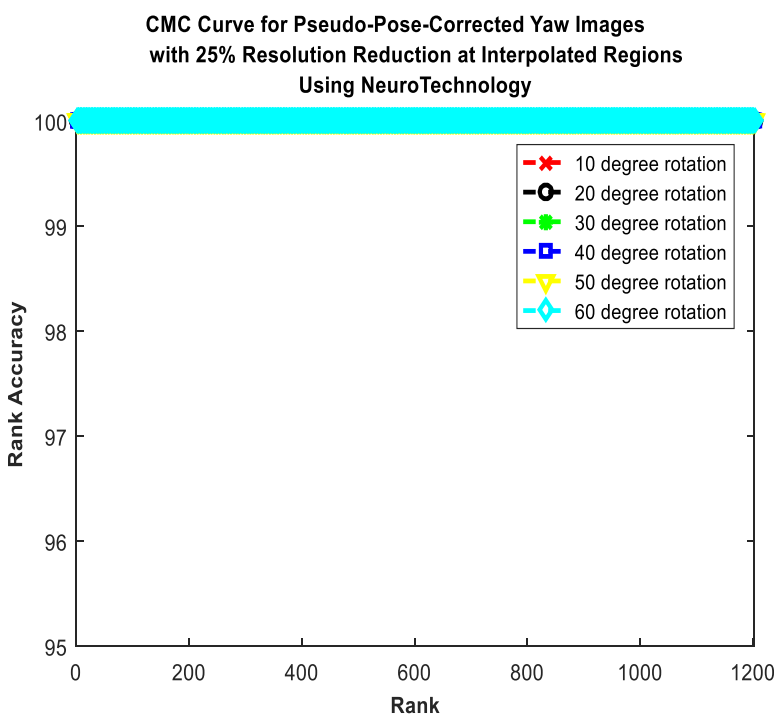

(b)

Figure 4.21 CMC of Pseudo Pose Corrected Yaw at 25\% Polygon Reduction Using Cognitec and NeuroTechnology Matchers, (a) CMC of Pseudo Pose Corrected Yaw at 25\% Polygon Reduction Using Cognitec FaceVACs, (b) CMC of Pseudo Pose Corrected Yaw at 25\% Polygon Reduction Using NeuroTechnology

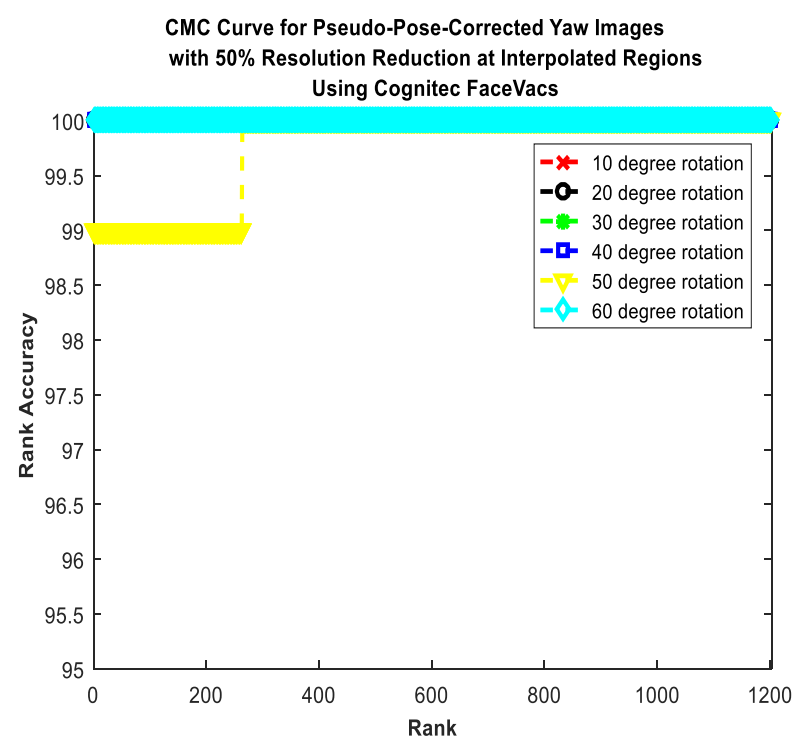

(a)

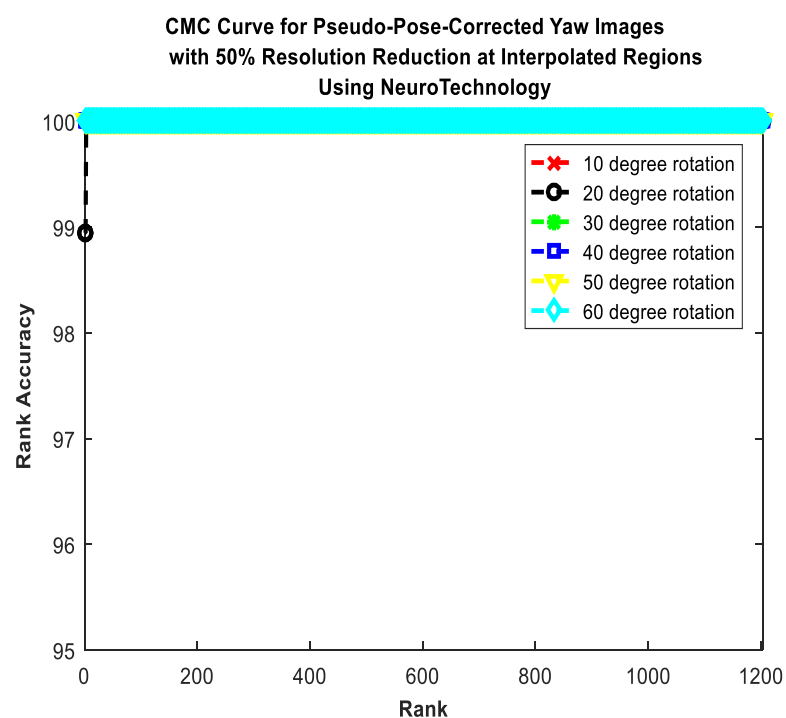

(b) 
Figure 4.22 CMC of Pseudo Pose Corrected Yaw at 50\% Polygon Reduction Using Cognitec and NeuroTechnology Matchers, (a) CMC of Pseudo Pose Corrected Yaw at 50\% Polygon Reduction Using Cognitec FaceVACs, (b) CMC of Pseudo Pose Corrected Yaw at 50\% Polygon Reduction Using NeuroTechnology

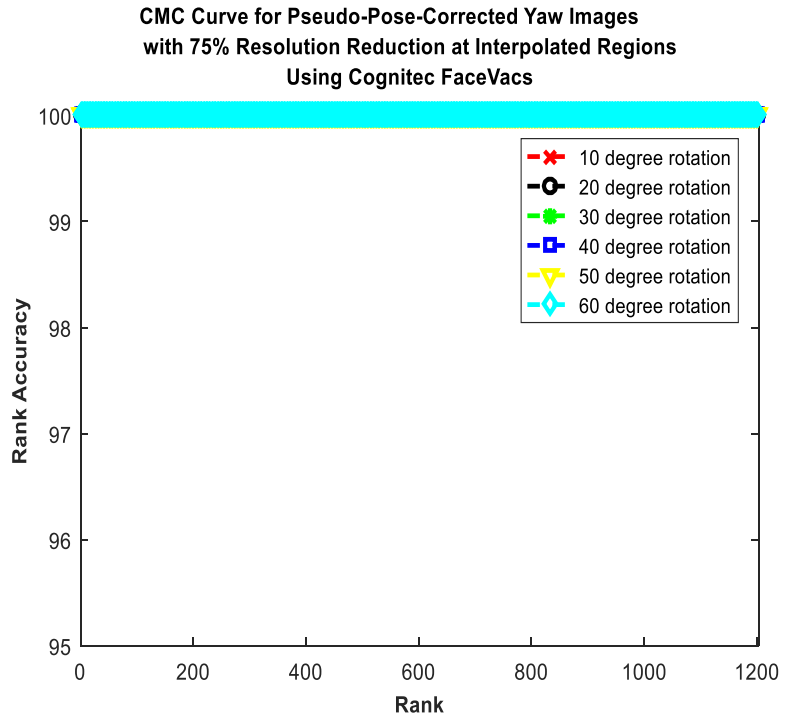

(a)

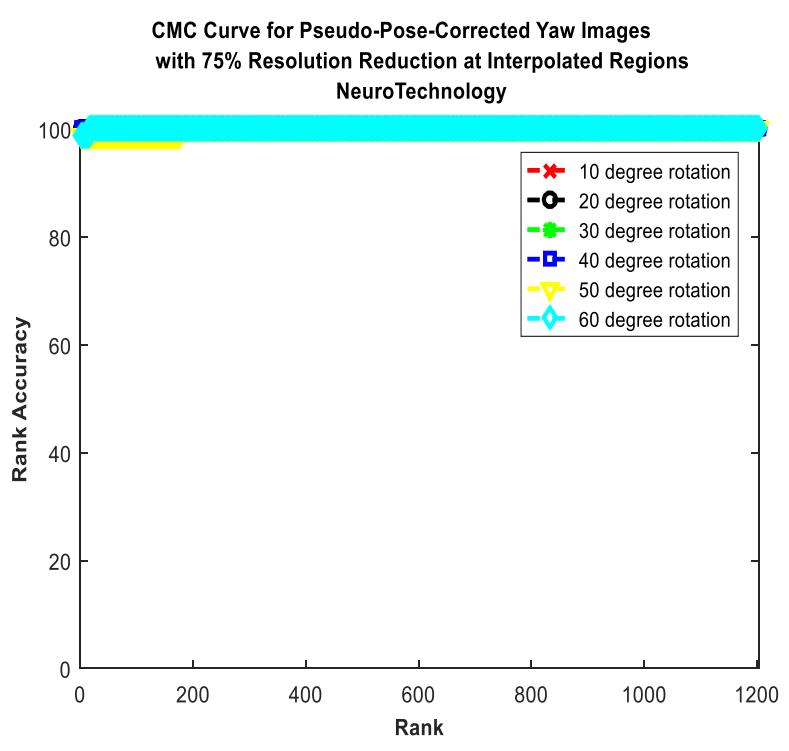

(b)

Figure 4.23 CMC of Pseudo Pose Corrected Yaw at 75\% Polygon Reduction Using Cognitec and NeuroTechnology Matchers, (a) CMC of Pseudo Pose Corrected Yaw at 75\% Polygon Reduction Using Cognitec FaceVACs, (b) CMC of Pseudo Pose Corrected Yaw at 75\% Polygon Reduction Using NeuroTechnology

When compared to the non-frontal and non-pose-corrected yaw images the performance of pseudo-pose corrected images has improved. As discussed earlier both Cognitec was able to detect and enroll only up to $50^{\circ}$ yaw rotations and NeuroTechnology was able to detect and enroll only up to $30^{\circ}$ yaw rotations whereas in pseudo-pose-corrected images all the yaw angles with higher degree of rotation and maximum polygon reduction were also detected and enrolled. But there is variation in match score with increased angle and polygon reduction as shown in the box plots below. 


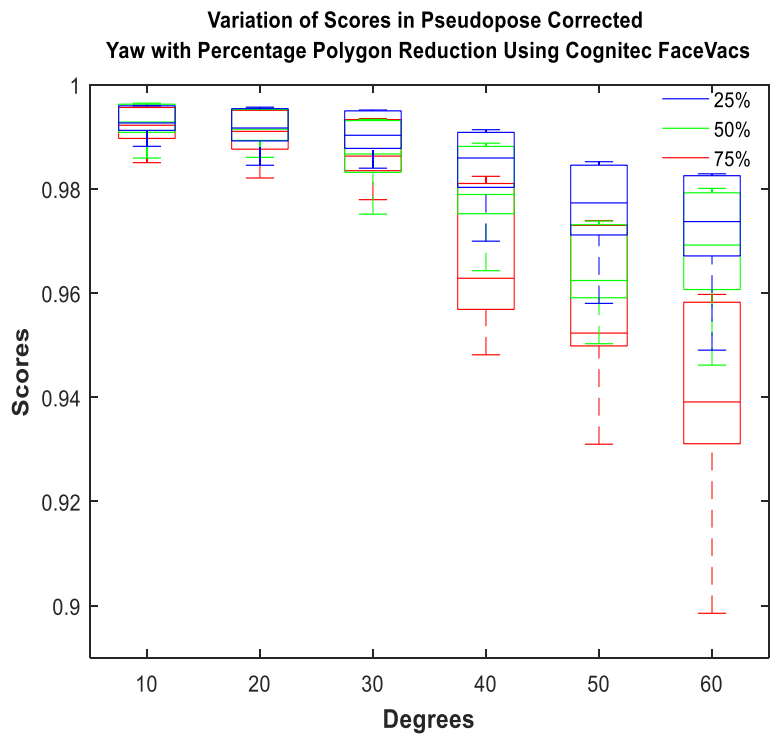

(a)

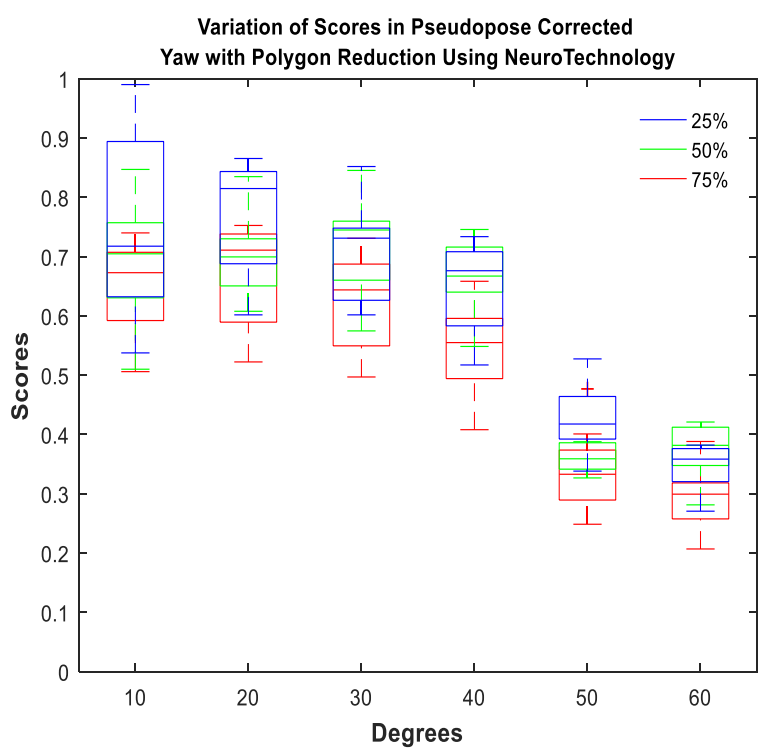

(b)

Figure 4.24 Match Score Distribution of Pseudo Pose Corrected Yaw with Varying Polygon Count, (a) Match Score Variations in Pseudo Pose Corrected Yaw Using Cognitec FaceVACs, (b) Match Score Variations in Pseudo Pose Corrected Yaw Using NeuroTechnology

The box plost shown in Figure 4.24 (a), (b) shows the variation in match score for pseudo-posecorrection images with varying polygon count for both Cognitec and NeuroTechnology matchers. The match score distribution of $25 \%, 50 \%$ and $75 \%$ polygon reduction in the box plots is indicated in blue, green and red respectively. This data shows that an increase in angle and reduction of polygon count causes the match score to decrease, even for pose-corrected images. Thus even pseudo-pose corrected images performed better over non-pose corrected images, there is drop in performance with increase in angle and polygon reduction.

\subsubsection{Performance of Pseudo Pose Corrected Pitch and Yaw Variations Images}

Performance of pseudo pose corrected pitch and yaw variations with polygons reduced at the interpolated regions is illustratively shown in the CMC curves in Figure 4.26, 4.27 and 4.28. The pseudo-pose corrected coupled pitch and yaw images has shown better performance than the nonpose-corrected which could detect and enroll only certain angular images. As discussed earlier from Table 4.5 Cognitec FaceVACs was able to detect and enroll 6 combinations of pitch and yaw 
and NeuroTechnology matcher was able to detect and enroll only 3 combinations out of 8 coupled pitch and yaw rotations. In pseudo-pose-corrected images all the coupled pitch and yaw angles with maximum polygon reduction were also detected and enrolled. But there is variation in match score with increased angle and polygon reduction as shown in the box plots in Figure 4.29 (a), (b).

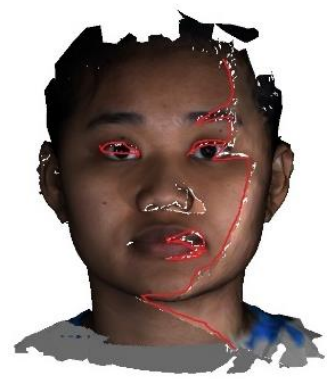

Pseudo Pose Corrected $30^{\circ}$ Pitch and $45^{\circ}$ Yaw at $75 \%$ Polygon Reduction

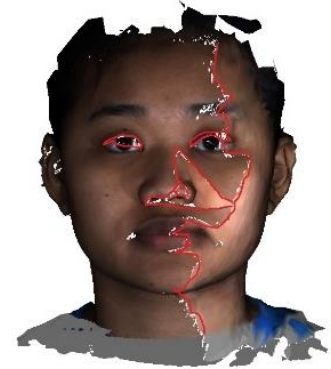

Pseudo Pose Corrected $30^{\circ}$ Pitch and $60^{\circ}$ Yaw at 75\% Polygon Reduction

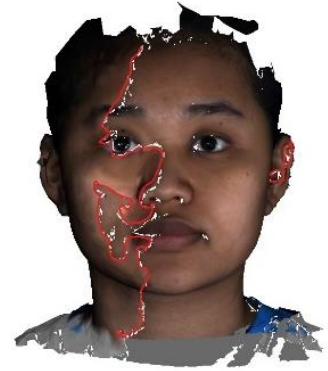

Pseudo Pose Corrected $30^{\circ}$ Pitch and $-60^{\circ}$ Yaw at 75\% Polygon Reduction

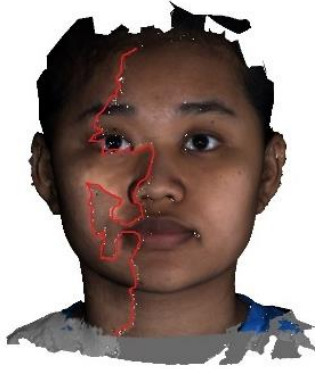

Pseudo Pose Corrected $30^{\circ}$ Pitch and $-60^{\circ}$ Yaw at $25 \%$ Polygon Reduction

Figure 4.25 Pseudo Pose Corrected Pitch and Yaw at Varying Polygon Reduction

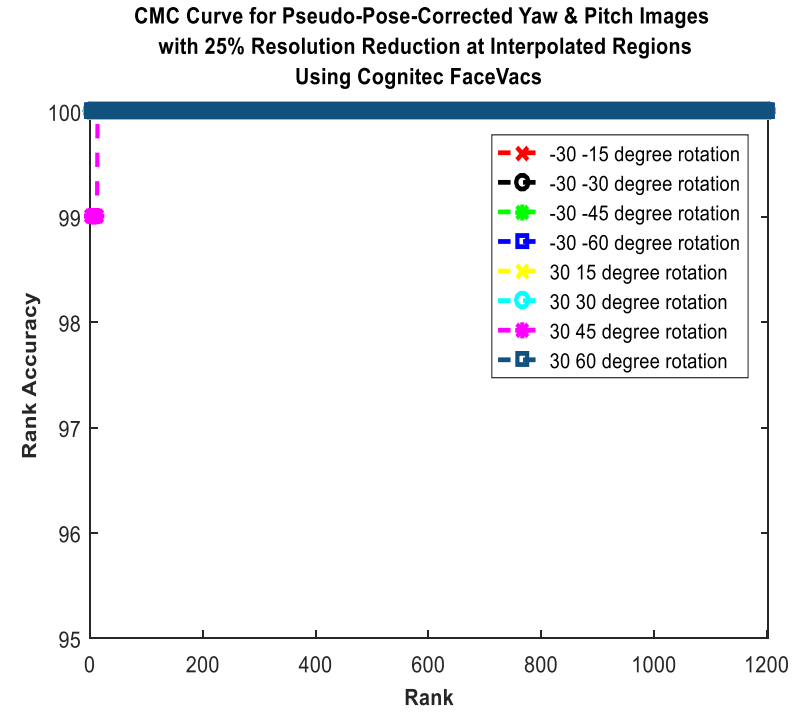

(a)

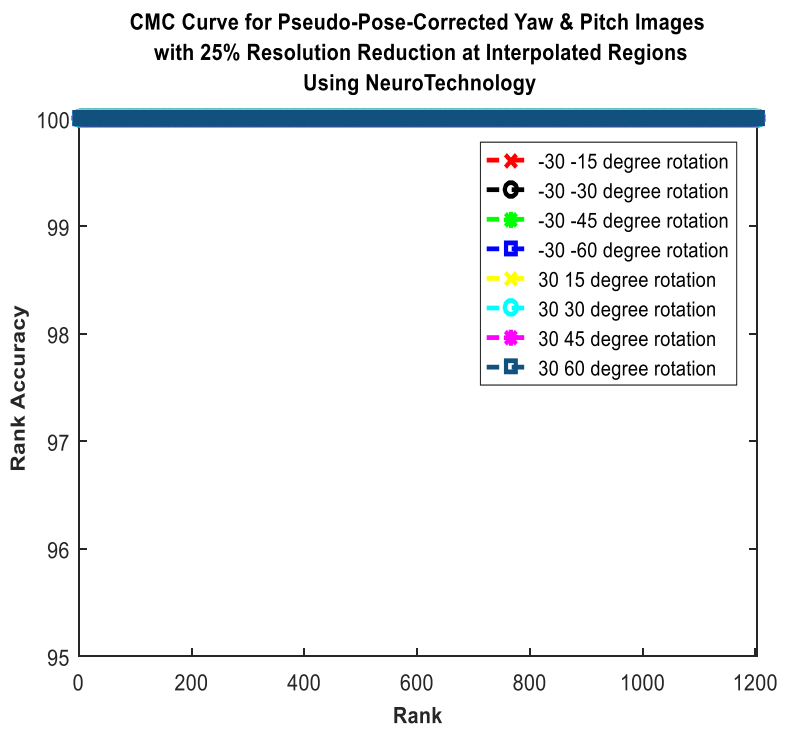

(b)

Figure 4.26 CMC of Pseudo Pose Corrected Pitch and Yaw at 25\% Polygon Reduction Using Cognitec and NeuroTechnology Matchers, (a) CMC of Pseudo Pose Corrected Pitch and Yaw at 
25\% Polygon Reduction Using Cognitec FaceVACs, (b) CMC of Pseudo Pose Corrected Pitch and Yaw at 25\% Polygon Reduction Using NeuroTechnology

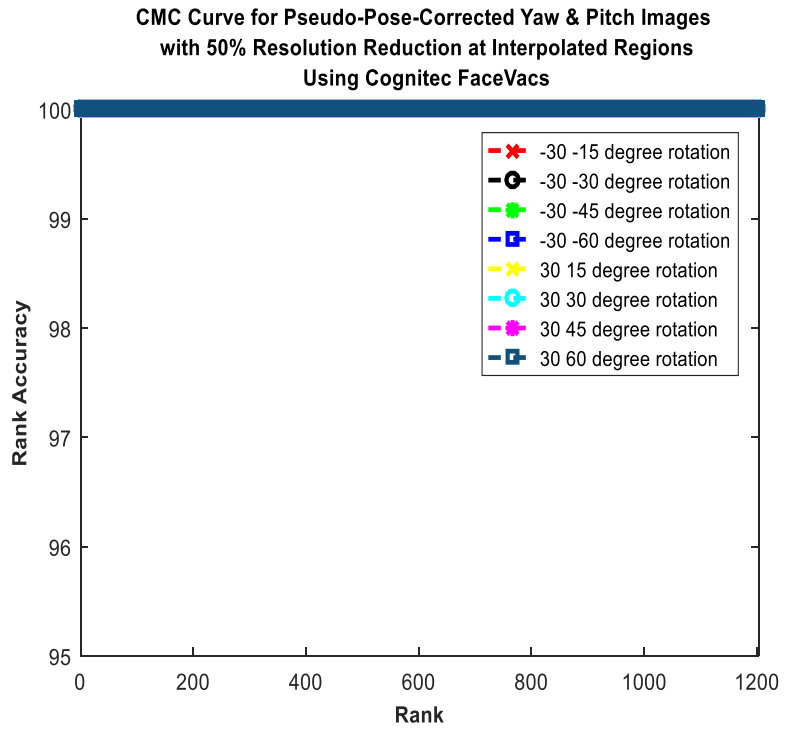

(a)

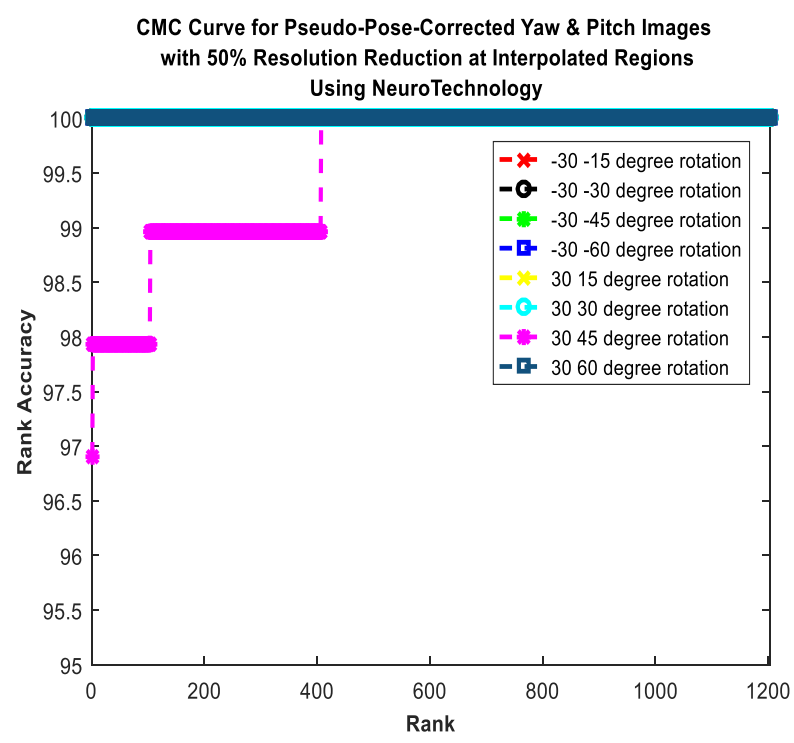

(b)

Figure 4.27 CMC of Pseudo Pose Corrected Pitch and Yaw at 50\% Polygon Reduction Using Cognitec and NeuroTechnology Matchers, (a) CMC of Pseudo Pose Corrected Pitch and Yaw at 50\% Polygon Reduction Using Cognitec FaceVACs, (b) CMC of Pseudo Pose Corrected Pitch and Yaw at 50\% Polygon Reduction Using NeuroTechnology 


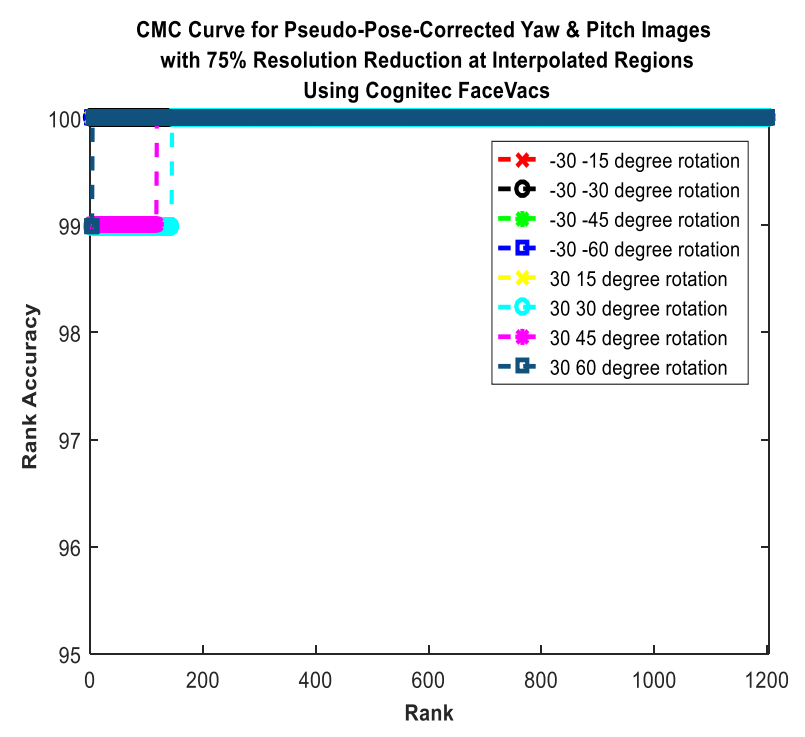

(a)

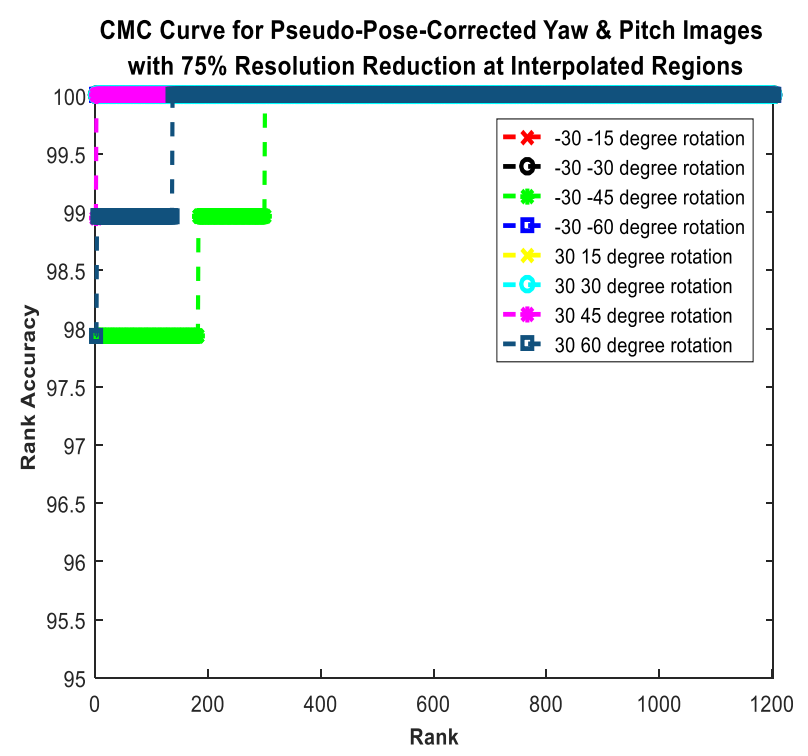

(b)

Figure 4.28 CMC of Pseudo Pose Corrected Pitch and Yaw at 75\% Polygon Reduction Using Cognitec and NeuroTechnology Matchers, (a) CMC of Pseudo Pose Corrected Pitch and Yaw at 75\% Polygon Reduction Using Cognitec FaceVACs, (b) CMC of Pseudo Pose Corrected Pitch and Yaw at 75\% Polygon Reduction Using NeuroTechnology

The box plost shown in Figure 4.29 (a), (b) shows the variation in match score for pseudo-posecorrection images with varying polygon count for both Cognitec and NeuroTechnology matchers. The match score distribution of $25 \%, 50 \%$ and $75 \%$ polygon reduction in the box plots is indicated in blue, green and red respectively. This data shows that an increase in angle and reduction of polygon count causes the match score to decrease, even for pose-corrected images. Thus even pseudo-pose corrected images performed better over non-pose corrected images, there is drop in performance with increase in angle and polygon reduction. 


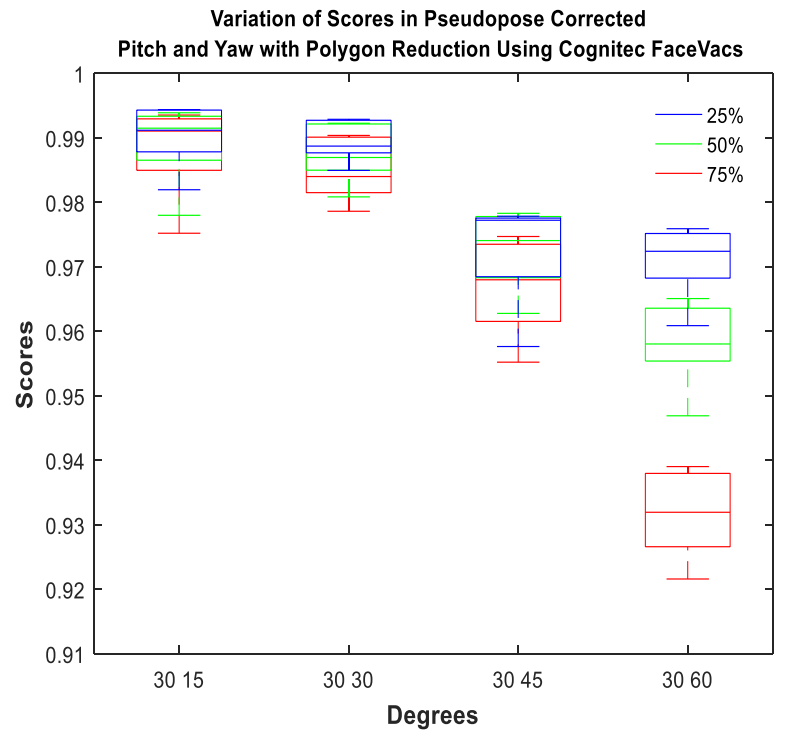

(a)

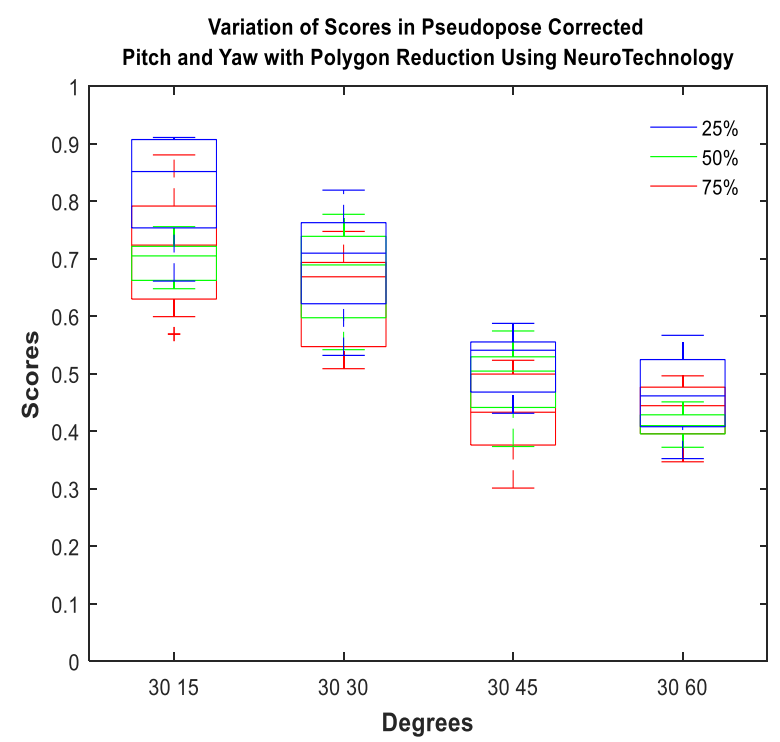

(b)

Figure 4.29 Match Score Distribution of Pseudo Pose Corrected Pitch and Yaw with Varying Polygon Count, (a) Match Score Variations in Pseudo Pose Corrected Pitch and Yaw Using Cognitec FaceVACs, (b) Match Score Variations in Pseudo Pose Corrected Pitch and Yaw Using NeuroTechnology

\subsection{Performance of Pose Corrected Images Using Cognitec DB Scan Pose Normalization}

In this project three different matchers are used Cognitec FaceVACs SDK, NeuroTechnology MegaMatcher and Cognitec DB Scan. Cognitec DB Scan has been mainly used for pose correction of non-frontal images. DB Scan has an inbuilt pose normalizing algorithm that generates a pose corrected image from a non-frontal image. The match performance of pose-corrected probe images against the gallery of 2D mug shot images has been evaluated using both Cognitec FaceVACs SDK and NeuroTechnology MegaMatcher. Results are presented in the form of CMC curves with the respective percentage of rank accuracy. 


\subsubsection{Pose Normalized Pitch Images}

Pose-varying pitch images are pose normalized using an inbuilt pose-normalization algorithm provided by Cognitec DB Scanner as explained in Chapter 3. These pose-corrected images are evaluated in a same manner as the non-pose corrected images by both the Cognitec FaceVACs and NeuroTechnology matchers and are assessed based on the match scores generated using CMC curves.

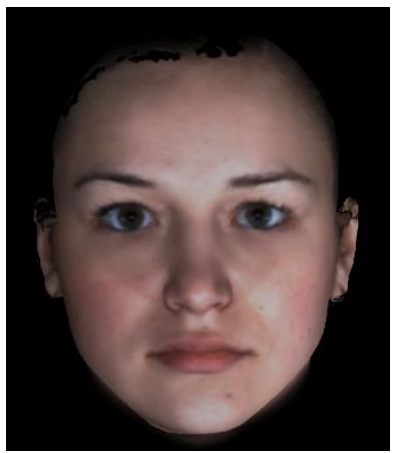

Pose Normalized $10^{\circ}$

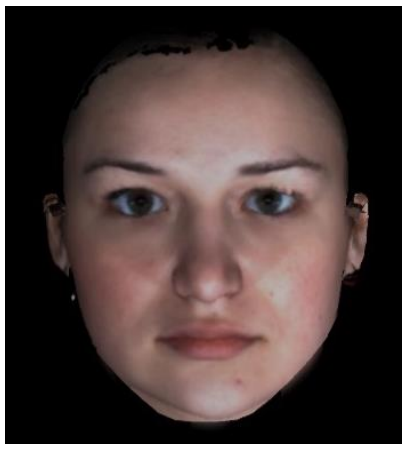

Pose Normalized $20^{\circ}$

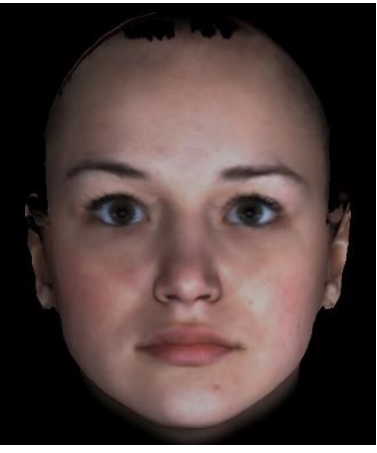

Pose Normalized $-10^{\circ}$

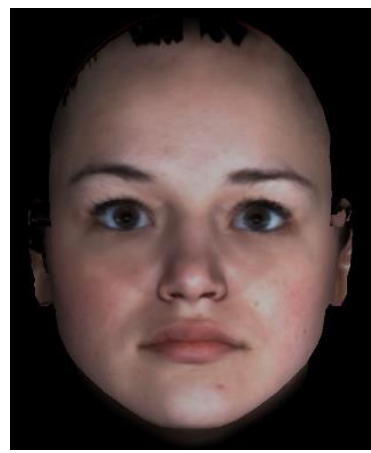

Pose Normalized $-20^{\circ}$

Pitch

Pitch Pitch Pitch

Figure 4.30 Pose Normalized Pitch Images Using Cognitec DB Scanner

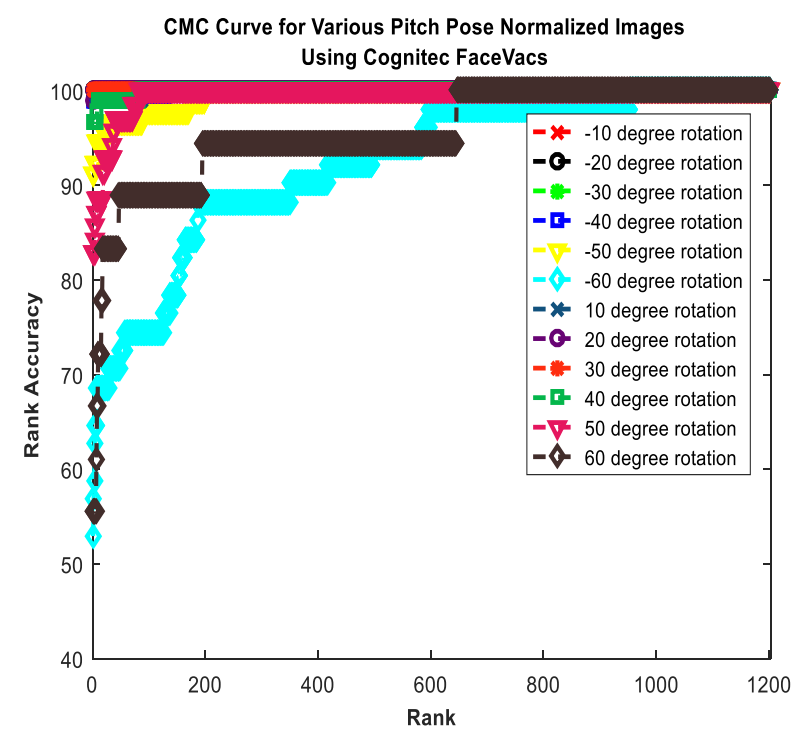

(a)

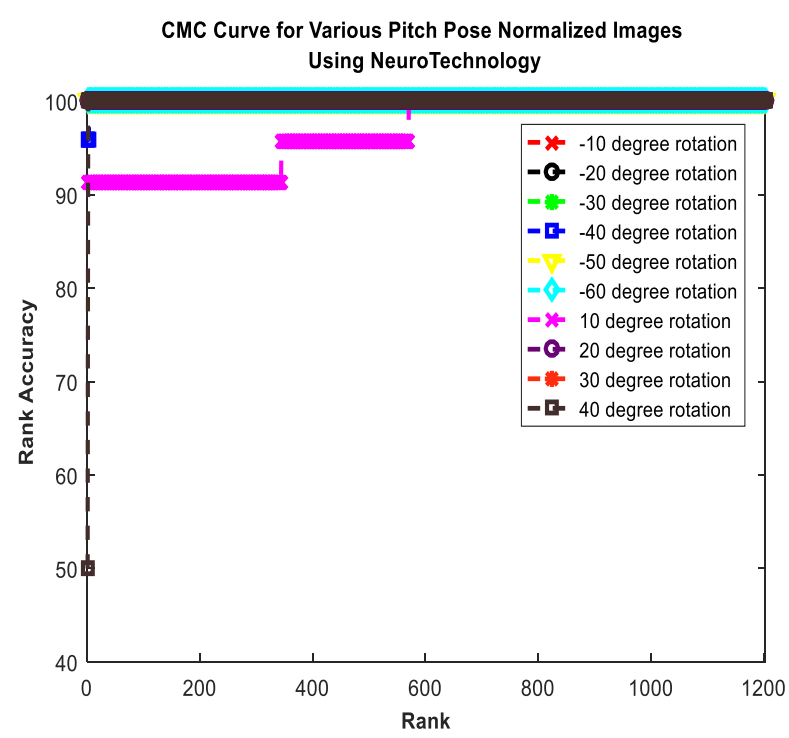

(b) 
Figure 4.31 CMC of Pose Normalized Pitch Using Cognitec and NeuroTechnology Matchers, (a) CMC of Pose Normalized Pitch Using Cognitec FaceVACs, (b) CMC of Pose Normalized Pitch Using NeuroTechnology

The CMC curves for pose-normalized pitch images are as shown in the Figure 4.31 (a), (b). In Figure 4.31 (a) as observed the Cognitec matcher was able to enroll all the angles which is a better compared to the uncorrected images and the rank-1 accuracy has decreased with the increase in degree of rotation where in the pseudo-pose-corrected case has better rank-1 accuracy for all the angles of pitch rotation. As seen in the Figure 4.31 (b) even after pose-correction the NeuroTechnology matcher was able to detect only up to $-50^{\circ}$ and $40^{\circ}$ rotation and has rank-1 accuracy values less than the accuracy of pseudo-pose-corrected images.

\subsubsection{Pose Normalized Yaw Images}

Similar method is implemented to generate pose-corrected yaw images from non-frontal yaw images using Cognitec DB Scanner. The pose-normalized yaw images using DB Scanner are shown in the Figure 4.32 .

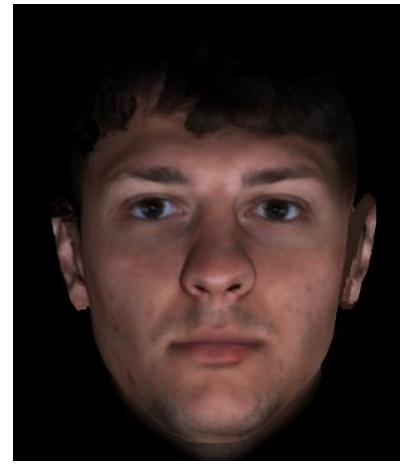

Pose Normalized $10^{\circ}$ Yaw

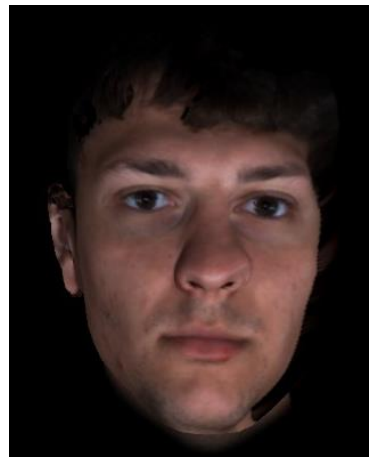

Pose Normalized $20^{\circ}$ Yaw

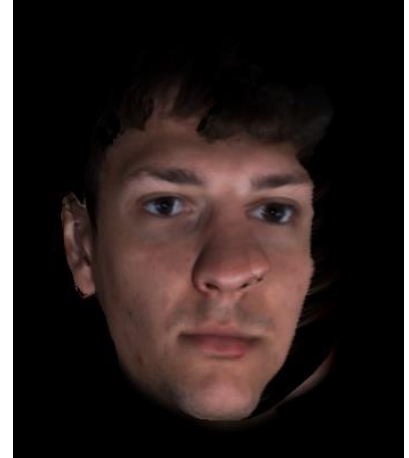

Pose Normalized $30^{\circ}$ Yaw

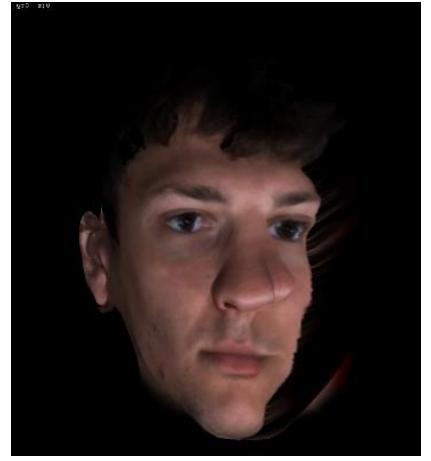

Pose Normalized $40^{\circ}$

Yaw

Figure 4.32 Pose Normalized Yaw Images Using Cognitec DB Scanner

The performance of pose-corrected images is evaluated in a similar manner as pitch images by generating the match score and are presented using CMC curves as shown in Figure 4.33 (a), (b). 


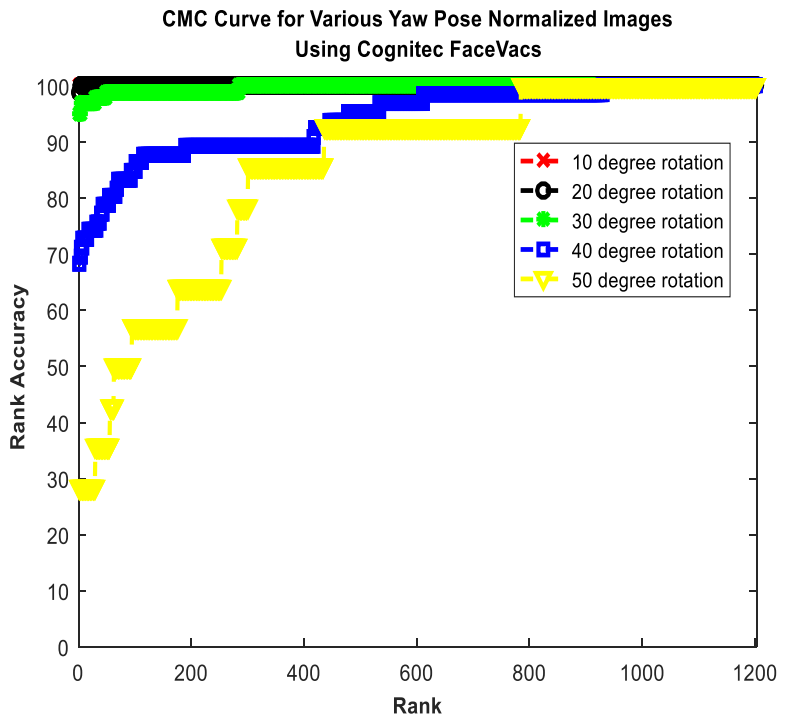

(a)

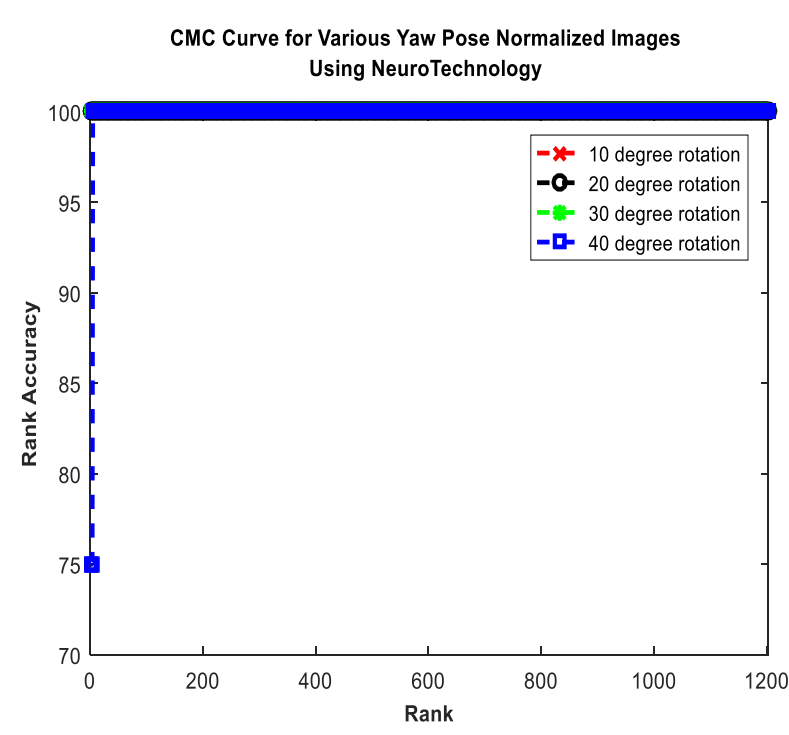

(b)

Figure 4.33 CMC of Pose Normalized Yaw Using Cognitec and NeuroTechnology Matchers, (a) CMC of Pose Normalized Yaw Using Cognitec FaceVACs, (b) CMC of Pose Normalized Yaw Using NeuroTechnology

In Figure 4.33 (a) as observed the Cognitec matcher was able to enroll only up to $50^{\circ}$ yaw rotation after pose-correction and when compared to the performance of pseudo-pose corrected images the rank-1 accuracy has considerably decreased with increase in angle. As seen in the Figure 4.33 (b) NeuroTechnology matcher was able to detect only up to $40^{\circ}$ yaw rotation after pose-correction where the pseudo-pose-correction method was able to detect and enroll all the angles and pseudopose-corrected images have better rank accuracy than the automatic pose-correction method.

\subsubsection{Pose Normalized Coupled Pitch and Yaw Images}

Pose-normalized coupled pitch and yaw angle images are generated in a similar way as pitch and yaw angles. The pose-corrected coupled pitch and yaw images are as shown in the Figure 4.32. The performance of these images is evaluated using the two commercial matchers similar to pitch and yaw images and is presented using CMC curves as shown in Figure 4.35 (a), (b). 


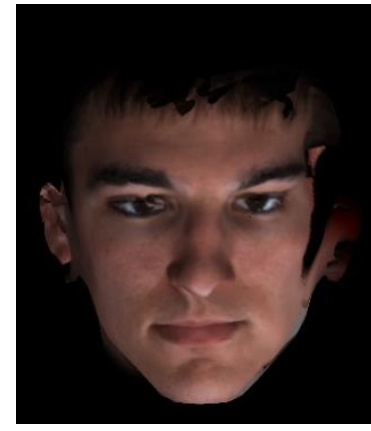

Pose Normalized $30^{\circ}$

Pitch and $15^{\circ}$ Yaw

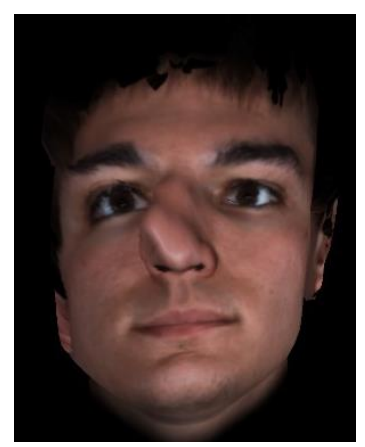

Pose Normalized $-30^{\circ}$

Pitch and $-15^{\circ}$ Yaw

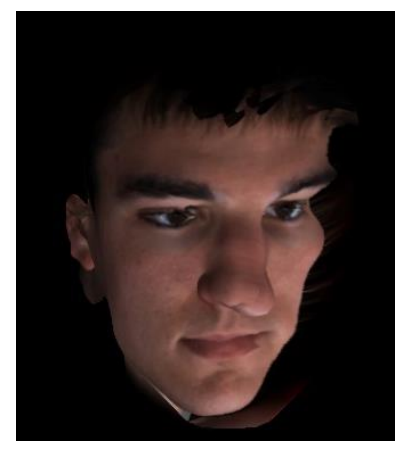

Pose Normalized $30^{\circ}$

Pitch and $30^{\circ}$ Yaw

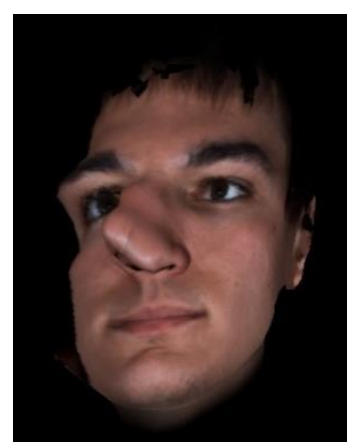

Pose Normalized $-30^{\circ}$

Pitch and $-30^{\circ}$ Yaw

Figure 4.34 Pose Normalized Pitch and Yaw Images Using Cognitec DB Scanner

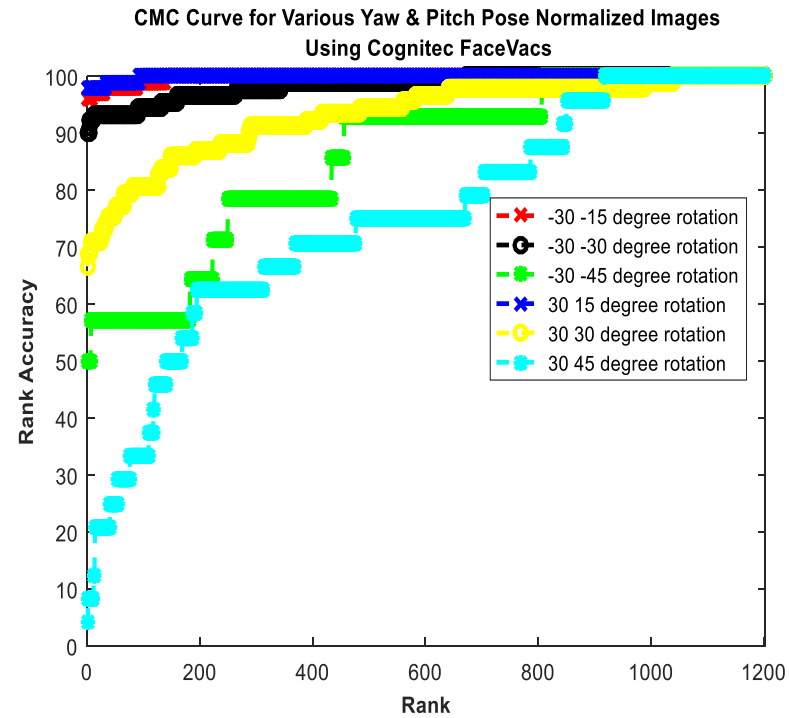

(a)

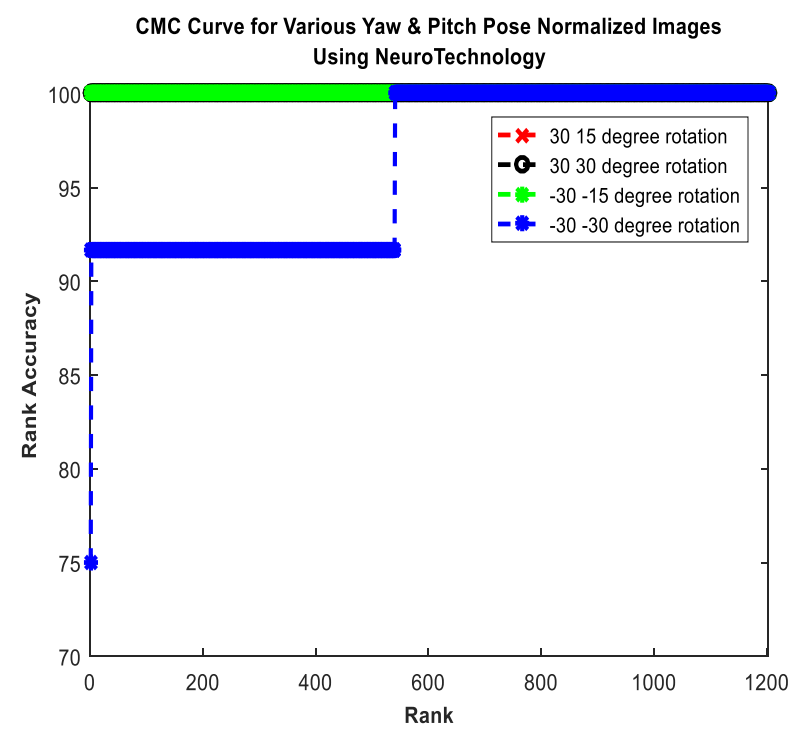

(b)

Figure 4.35 CMC of Pose Normalized Pitch and Yaw Using Cognitec and NeuroTechnology Matchers, (a) CMC of Pose Normalized Pitch and Yaw Using Cognitec FaceVACs, (b) CMC of Pose Normalized Pitch and Yaw Using NeuroTechnology

From Figure 4.35 (a), (b) Cognitec could detect and enroll 5 combination of coupled pitch and yaw angle rotations and NeuroTechnology could detect and enroll 4 combinations out of 8 after posecorrection. This is a better when compared to the uncorrected image enrollment but the pseudopose correction method has a better enrollment rate as it has could detect and enroll all 8 
combination of coupled pitch and yaw variations with three different polygon reductions. The pseudo-pose-correction method has a better rank accuracy when compared to pose-correction method as, in this method the rank-1 accuracy has decreased for greater degrees of coupled pitch and yaw. 


\section{Chapter 5 Conclusions and Future Work}

This chapter provides a summary of conclusions for this work, as well as a discussion of further advancements that can be implemented in future.

\subsection{Conclusions}

The goal of this work is to evaluate the effectiveness of commercial pose-correction software using 2D non-frontal pose images generated using 3D facial scans.

Match scores are the main metric deployed for evaluation of two commercial face matchers: NeuroTechnology MegaMatcher and Cognitec FaceVACs. For both matchers, four different matching experiments were implemented using frontal, non-frontal, and pose-corrected images. Those four experiments are:

1. Frontal (probe) to Frontal (gallery) images, to get a sense of the baseline match score distribution for ideal matching circumstances

2. Non-ideal pose-varying (probe) to frontal (gallery)

3. Pseudo-pose-corrected (probe) to frontal (gallery)

4. Auto-pose-corrected (probe) to frontal (gallery)

The generated match scores are examined using cumulative match characteristic curves (CMC) and boxplots. In addition to the pseudo-pose-correction method, an automatic pose correction method is implemented, specifically, Cognitec FaceVACs DB Scan. In DB Scan, non-frontal face images are pose-normalized to frontal faces and matched against the gallery set. As with attempts to enroll the non-frontal images, the rate of enrollment decreased with an increase in the degree of rotation, resulting in fewer images in each probe set. (Varying size of probe sets in which the number of images decreased with increase in angle also resulted in a 100 percent accuracy in which variations can be observed in the match score distribution from the box plots).

The NeuroTechnology matcher could detect (i.e. enroll) non-frontal face images with up to $40^{\circ}$ and $-50^{\circ}$ pitch rotations, up to $30^{\circ}$ yaw rotations, and coupled pitch/yaw angle rotations of $30^{\circ} 15^{\circ}$ and $-30^{\circ}-15$. Cognitec FaceVACs has detected faces with up to $50^{\circ}$ and $-60^{\circ}$ pitch rotation, up to 
$40^{\circ}$ yaw rotations, and coupled pitch/yaw angle rotations of $30^{\circ} 15^{\circ}, 30^{\circ} 30^{\circ},-30^{\circ}-15^{\circ}$ and $-30^{\circ}-$ $30^{\circ}$.

For a set of probe images comprised of pseudo-pose-corrected faces with regions of lower resolution interpolate data in occluded regions, all the images were successfully detected and enrolled by the matchers. When matching these pseudo-pose-corrected images against the gallery of frontal mugshot images, the matching performance has decreased with a decrease in the resolution of the recovered occluded areas, achieved in this work by reducing the number of polygons in occluded regions of the 3D image. For example, images with $25 \%$ polygon reduction in occluded regions have a better performance than images of the same individual with $75 \%$ polygon reduction in the same regions.

The rank-1 accuracy performance of the list of experiments conducted in this work as described above are given in the following tables. The rank-1 accuracy for the baseline performance of frontal (probe) to frontal (gallery) is $100 \%$.

Table 5.1 and 5.2 gives the rank-1 accuracy of uncorrected, pseudo-pose-corrected and posecorrected images for pitch variations in both Cognitec and NeuroTechnology matchers. As observed from the tables pseudo-pose-corrected images has an overall rank-1 accuracy of 100\% and for the uncorrected images greater angle rotations are not detected by the NeuroTechnology matcher where as Cognitec Matcher could detect and enroll all the angular variations but has a low rank-1 accuracy of $65.79 \%, 25 \%, 76.59 \%, 35.89 \%$ and $11.11 \%$ for larger angles $50^{\circ}, 60^{\circ},-40^{\circ}$, $50^{\circ}$ and $-60^{\circ}$ respectively. The pose-corrected method even after pose-correction could not detect certain angles like $50^{\circ}$ and $60^{\circ}$ using NeuroTechnology matcher. Using Cognitec could detect all the angles but has a low rank accuracy for large angles $50^{\circ}, 60^{\circ}$ and $-60^{\circ}$. 
Table 5.1 Rank Accuracies of Various Pitch angle for Pseudo-Pose Corrected and Pose-Varying for Cognitec FaceVACs

\begin{tabular}{|c|c|c|c|c|c|}
\hline \multirow[b]{2}{*}{ Pitch Angle } & \multirow[b]{2}{*}{$\begin{array}{c}\text { Rank-1 } \\
\text { Accuracy } \\
\text { Uncorrected }\end{array}$} & \multicolumn{3}{|c|}{ Pseudo-Pose Correction Cases-Pitch Angle Cognitec } & \multirow[b]{2}{*}{$\begin{array}{c}\text { Rank-1 } \\
\text { Accuracy } \\
\text { Pose-Normalized }\end{array}$} \\
\hline & & $\begin{array}{c}\text { Rank-1 } \\
\text { Accuracy } \\
\text { 25\% Polygon } \\
\text { Count Reduced }\end{array}$ & $\begin{array}{c}\text { Rank-1 } \\
\text { Accuracy } \\
\text { 50\% Polygon } \\
\text { Count Reduced }\end{array}$ & $\begin{array}{c}\text { Rank-1 } \\
\text { Accuracy } \\
\text { 75\% Polygon } \\
\text { Count Reduced }\end{array}$ & \\
\hline $10^{\circ}$ & 100 & 100 & 100 & 100 & 98.98 \\
\hline $20^{\circ}$ & 100 & 100 & 100 & 100 & 98.95 \\
\hline $30^{\circ}$ & 100 & 100 & 100 & 100 & 100 \\
\hline $40^{\circ}$ & 97.64 & 100 & 100 & 100 & 96.7 \\
\hline $50^{\circ}$ & 65.79 & 100 & 100 & 100 & 82.85 \\
\hline $60^{\circ}$ & 25 & 100 & 100 & 97.93 & 55.55 \\
\hline$-10^{\circ}$ & 100 & 100 & 100 & 100 & 98.98 \\
\hline$-20^{\circ}$ & 100 & 100 & 100 & 100 & 100 \\
\hline$-30^{\circ}$ & 96.96 & 100 & 100 & 100 & 100 \\
\hline$-40^{\circ}$ & 76.59 & 100 & 100 & 98.98 & 98.95 \\
\hline$-50^{\circ}$ & 35.89 & 100 & 98.98 & 98.09 & 91.3 \\
\hline$-60^{\circ}$ & 11.11 & 100 & 99.89 & 96.91 & 52.94 \\
\hline
\end{tabular}

Table 5.2 Rank Accuracies of Various Pitch angle for Pseudo-Pose Corrected and Pose-Varying for NeuroTechnology

\begin{tabular}{|c|c|c|c|c|c|}
\hline \multirow{2}{*}{} & \multirow{4}{*}{ Pitch Angle } & \multicolumn{2}{|c|}{ Pseudo-Pose Correction Cases-Pitch Angle } & \\
\cline { 3 - 5 } & $\begin{array}{c}\text { Rank-1 } \\
\text { Accuracy } \\
\text { Uncorrected }\end{array}$ & $\begin{array}{c}\text { Rank-1 } \\
\text { Accuracy } \\
25 \% \text { Polygon } \\
\text { Count Reduced }\end{array}$ & $\begin{array}{c}\text { Rank-1 } \\
\text { Accuracy } \\
\text { 50\% Polygon } \\
\text { Count Reduced }\end{array}$ & $\begin{array}{c}\text { Rank-1 } \\
\text { Accuracy } \\
\text { 75\% Polygon } \\
\text { Count Reduced }\end{array}$ & $\begin{array}{c}\text { Rank-1 } \\
\text { Accuracy Pose } \\
\text { Normalized }\end{array}$ \\
\hline $10^{\circ}$ & 100 & 100 & 100 & 100 & 91.3 \\
\hline $20^{\circ}$ & 100 & 100 & 100 & 100 & 100 \\
\hline $30^{\circ}$ & 92.5 & 100 & 100 & 100 & 100 \\
\hline $40^{\circ}$ & 71.42 & 98.96 & 100 & 100 & 50 \\
\hline $50^{\circ}$ & - & 98.96 & 100 & 98.96 & - \\
\hline $60^{\circ}$ & - & 98.96 & 100 & 97.87 & - \\
\hline
\end{tabular}




\begin{tabular}{|c|c|c|c|c|c|}
\hline$-10^{\circ}$ & 100 & 100 & 100 & 100 & 100 \\
\hline$-20^{\circ}$ & 93.3 & 100 & 100 & 100 & 100 \\
\hline$-30^{\circ}$ & 82.85 & 100 & 100 & 100 & 100 \\
\hline$-40^{\circ}$ & $\mathbf{2 6 . 6 6}$ & 100 & 100 & 98.96 & 95.83 \\
\hline$-50^{\circ}$ & $\mathbf{1 4 . 2 8}$ & 100 & 98.97 & 98.95 & 100 \\
\hline$-60^{\circ}$ & - & 98.96 & 98.96 & 98.90 & 100 \\
\hline
\end{tabular}

Table 5.3 and 5.4 gives the rank-1 accuracy of uncorrected, pseudo-pose-corrected and posecorrected images for yaw variations in both Cognitec and NeuroTechnology matchers. As observed from the tables pseudo-pose-corrected images has an overall rank-1 accuracy of 100\% in this case also and for the uncorrected images yaw angle greater than $30^{\circ}$ was not detected by the NeuroTechnology matcher where as Cognitec Matcher was unable to detect only $60^{\circ}$ rotation. However, for uncorrected images it has achieved 100\% rank-1 accuracy using Cognitec matcher and has shown a decreasing trend using NeuroTechnology matcher. The pose-corrected method even after pose-correction was unable to detect angles greater than $40^{\circ}$ using NeuroTechnology matcher and was unable to detect only $60^{\circ}$ rotation using Cognitec. But has a low rank-1 accuracy for greater angles.

Table 5.3 Rank Accuracies of Various Yaw angle for Pseudo-Pose Corrected and Pose-Varying for Cognitec FaceVACs

\begin{tabular}{|c|c|c|c|c|c|}
\hline \multirow{2}{*}{ Yaw } & & \multicolumn{2}{|c|}{ Pseudo-Pose Correction Cases-Yaw Angle Cognitec } & \\
\cline { 3 - 5 } & $\begin{array}{c}\text { Rank-1 } \\
\text { Accuracy } \\
\text { Uncorrected }\end{array}$ & $\begin{array}{c}\text { Rank-1 } \\
\text { Accuracy } \\
25 \% \text { Polygon } \\
\text { Count Reduced }\end{array}$ & $\begin{array}{c}\text { Rank-1 } \\
\text { Accuracy } \\
\text { 50\% Polygon } \\
\text { Count Reduced }\end{array}$ & $\begin{array}{c}\text { Rank-1 } \\
\text { Accuracy } \\
75 \% \text { Polygon } \\
\text { Count Reduced }\end{array}$ & Rank-1 Accuracy \\
Pose-Normalized \\
\hline $10^{\circ}$ & 100 & 100 & 100 & 100 & \\
\hline $20^{\circ}$ & 100 & 100 & 100 & 100 & 99 \\
\hline $30^{\circ}$ & 100 & 100 & 100 & 100 & 95 \\
\hline $40^{\circ}$ & 100 & 100 & 100 & 100 & 68.18 \\
\hline $50^{\circ}$ & 100 & 100 & 100 & 100 & $\mathbf{2 8 . 5 7}$ \\
\hline $60^{\circ}$ & - & 100 & 100 & 100 & - \\
\hline
\end{tabular}


Table 5.4 Rank Accuracies of Various Yaw angle for Pseudo-Pose Corrected and Pose-Varying for NeuroTechnology

\begin{tabular}{|c|c|c|c|c|c|}
\hline \multirow[b]{2}{*}{ Yaw } & \multirow[b]{2}{*}{$\begin{array}{c}\text { Rank-1 } \\
\text { Accuracy } \\
\text { Uncorrected }\end{array}$} & \multicolumn{3}{|c|}{$\begin{array}{c}\text { Pseudo-Pose Correction Cases-Yaw Angle } \\
\text { NeuroTechnology }\end{array}$} & \multirow[b]{2}{*}{$\begin{array}{l}\text { Rank-1 Accuracy } \\
\text { Pose Normalized }\end{array}$} \\
\hline & & $\begin{array}{c}\text { Rank-1 } \\
\text { Accuracy } \\
\text { 25\% Polygon } \\
\text { Count Reduced }\end{array}$ & $\begin{array}{c}\text { Rank-1 } \\
\text { Accuracy } \\
\text { 50\% Polygon } \\
\text { Count Reduced }\end{array}$ & $\begin{array}{c}\text { Rank-1 } \\
\text { Accuracy } \\
\text { 75\% Polygon } \\
\text { Count Reduced }\end{array}$ & \\
\hline $10^{\circ}$ & 100 & 100 & 100 & 100 & 100 \\
\hline $20^{\circ}$ & 90.62 & 100 & 98.94 & 100 & 100 \\
\hline $30^{\circ}$ & 60 & 100 & 100 & 100 & 100 \\
\hline $40^{\circ}$ & - & 100 & 100 & 100 & 75 \\
\hline $50^{\circ}$ & - & 100 & 100 & 98.95 & - \\
\hline $60^{\circ}$ & - & 100 & 100 & 98.94 & - \\
\hline
\end{tabular}

Table 5.5 and 5.5 gives the rank-1 accuracy of uncorrected, pseudo-pose-corrected and posecorrected images for coupled pitch and yaw variations in both Cognitec and NeuroTechnology matchers. As observed from the tables pseudo-pose-corrected images has an overall rank-1 accuracy of $100 \%$, which is been observed for all three polygon variations and for the uncorrected images 6 combinations $\left(30^{\circ} 15^{\circ}, 30^{\circ} 30^{\circ}, 30^{\circ} 45^{\circ},-30^{\circ}-15^{\circ},-30^{\circ}-30^{\circ},-30^{\circ}-45^{\circ}\right)$ of coupled pitch and yaw were detected and enrolled out of 8 combinations using Cognitec matcher whereas NeuroTechnology matcher could detect only 3 combinations $\left(30^{\circ} 15^{\circ},-30^{\circ}-15^{\circ},-30^{\circ}-30^{\circ}\right)$ out of 8 coupled pitch and yaw combinations. However, for uncorrected images the rank-1 accuracy using Cognitec matcher has better performance than the NeuroTechnology matcher which has very low rank-1 accuracy values for the detected angles. The pose-corrected method even after posecorrection could detect only 4 combinations using NeuroTechnology matcher and using Cognitec it could detect only 6 combinations. The rank-1 accuracy values has a decreasing trend with increase in angle whereas using NeuroTechnology matcher only $-30^{\circ}-30^{\circ}$, have a $75 \%$ rank-1 accuracy while others have a $100 \%$ rank-1 accuracy. 
Table 5.5 Rank Accuracies of Various Coupled Pitch/Yaw angle for Pseudo-Pose Corrected and Pose-Varying for Cognitec FaceVACs

\begin{tabular}{|c|c|c|c|c|c|}
\hline \multirow[b]{2}{*}{$\begin{array}{c}\text { Coupled } \\
\text { Pitch/Yaw } \\
\text { Angle }\end{array}$} & \multirow[b]{2}{*}{$\begin{array}{c}\text { Rank-1 } \\
\text { Accuracy } \\
\text { Uncorrected }\end{array}$} & \multicolumn{3}{|c|}{$\begin{array}{c}\text { Pseudo-Pose Correction Cases-Coupled Pitch/Yaw } \\
\text { Angle Cognitec }\end{array}$} & \multirow[b]{2}{*}{$\begin{array}{c}\text { Rank-1 } \\
\text { Accuracy } \\
\text { Pose-Normalized }\end{array}$} \\
\hline & & $\begin{array}{c}\text { Rank-1 } \\
\text { Accuracy } \\
\text { 25\% Polygon } \\
\text { Count Reduced }\end{array}$ & $\begin{array}{c}\text { Rank-1 } \\
\text { Accuracy } \\
\text { 50\% Polygon } \\
\text { Count Reduced }\end{array}$ & $\begin{array}{c}\text { Rank-1 } \\
\text { Accuracy } \\
\text { 75\% Polygon } \\
\text { Count Reduced }\end{array}$ & \\
\hline $30^{\circ} 15^{\circ}$ & 98.95 & 100 & 100 & 100 & 97.95 \\
\hline $30^{\circ} 30^{\circ}$ & 94.59 & 100 & 100 & 98.99 & 66.66 \\
\hline $30^{\circ} 45^{\circ}$ & 100 & 99 & 100 & 99 & 4.166 \\
\hline $30^{\circ} 60^{\circ}$ & - & 100 & 100 & 98.99 & $=$ \\
\hline$-30^{\circ}-15^{\circ}$ & 93.88 & 100 & 100 & 100 & 95.95 \\
\hline$-30^{\circ}-30^{\circ}$ & 93.4 & 100 & 100 & 100 & 90 \\
\hline$-30^{\circ}-45^{\circ}$ & 87.5 & 100 & 100 & 100 & 50 \\
\hline$-30^{\circ}-60^{\circ}$ & - & 100 & 100 & 100 & - \\
\hline
\end{tabular}

Table 5.6 Rank Accuracies of Various Coupled Pitch/Yaw angle for Pseudo-Pose Corrected and Pose-Varying for NeuroTechnology

\begin{tabular}{|c|c|c|c|c|c|}
\hline \multirow[b]{2}{*}{$\begin{array}{c}\text { Coupled } \\
\text { Pitch/Yaw } \\
\text { Angle }\end{array}$} & \multirow[b]{2}{*}{$\begin{array}{c}\text { Rank-1 } \\
\text { Accuracy } \\
\text { Uncorrected }\end{array}$} & \multicolumn{3}{|c|}{$\begin{array}{l}\text { Pseudo-Pose Correction Cases-Coupled Pitch/Yaw } \\
\text { Angle Neurotechnology }\end{array}$} & \multirow[b]{2}{*}{$\begin{array}{c}\text { Rank-1 } \\
\text { Accuracy Pose } \\
\text { Normalized }\end{array}$} \\
\hline & & $\begin{array}{c}\text { Rank-1 } \\
\text { Accuracy } \\
\text { 25\% Polygon } \\
\text { Count Reduced }\end{array}$ & $\begin{array}{c}\text { Rank-1 } \\
\text { Accuracy } \\
\text { 50\% Polygon } \\
\text { Count Reduced }\end{array}$ & $\begin{array}{c}\text { Rank-1 } \\
\text { Accuracy } \\
\text { 75\% Polygon } \\
\text { Count Reduced }\end{array}$ & \\
\hline $30^{\circ} 15^{\circ}$ & 76.19 & 100 & 100 & 100 & 100 \\
\hline $30^{\circ} 30^{\circ}$ & - & 100 & 100 & 100 & 100 \\
\hline $30^{\circ} 45^{\circ}$ & - & 100 & 96.91 & 98.94 & $=$ \\
\hline $30^{\circ} 60^{\circ}$ & - & 100 & 100 & 97.93 & - \\
\hline$-30^{\circ}-15^{\circ}$ & 60 & 100 & 100 & 100 & 100 \\
\hline$-30^{\circ}-30^{\circ}$ & 50 & 100 & 100 & 100 & 75 \\
\hline$-30^{\circ}-45^{\circ}$ & - & 100 & 100 & 97.93 & - \\
\hline$-30^{\circ}-60^{\circ}$ & - & 100 & 100 & 100 & - \\
\hline
\end{tabular}


Thus, these results can be interpreted as follows:

- The number of subjects considered in this work is relatively small, even considering that there were 8-16 image samples for each of the 100 participants.

- All the original images were acquired under controlled conditions: neutral pose, expression and uniform illumination.

Tables 5.1, 5.2, 5.3, 5.4, 5.5 and 5.6 shows the rank-1 match accuracy of pitch, yaw and coupled pitch/yaw angles for the non-corrected, pseudo-pose-corrected and pose-corrected (normalized) images. As can be observed, the match accuracy decreases with an increase of facial rotation for all cases and the rank accuracy for pseudo-pose corrected images has an overall better performance than the pose-normalized images. Observing the accuracy for different experiments the Cognitec matcher has a better efficiency over Neurotechnology matcher in case of images with pose variation. The presence of inbuild pose-correction algorithm would have promoted in a better performance of Cognitec in pose-varying images than the NeuroTechnology matcher which is not designed for pose-varying applications.

The Cognitec FaceVACs, which includes the SDK and DB Scan, costs around \$30,000 U.S dollars and NeuroTechnology Mega Matcher costs around \$5000 U.S dollars. Therefore, it depends on end-user or the client to choose the product as per the requirement of the application which would assist in a better analysis of the data.

\subsection{Future Work}

This study can be extended in several ways. In this project, a separate set of data has been generated using 3D models which can be used for further study of pose varying face images. Custom, noncommercial pose-correction algorithms can be designed to evaluate this data. As a very small uniform set of data has been used in this project, which resulted in 100\% percent match accuracy for some of the pose-correction experiments. Experimentation on a lager set of data with images acquired under different conditions could result in varied results. This experimentation would make this study pertinent to the real-world facial biometric applications. 


\section{References}

[1] A. T. P. Georgios Goudelis, "Intelligent Multimedia Analysis for Emerging," Dept. of Informatics, Aristotle University of Thessaloniki,Greece.

[2] A. A. R. K. N. Anil K. Jain, Introduction to Biomerics, Springer, 2011.

[3] "Fingerprint Recognition".

[4] P. F. A. A. R. ANil K. Jain, Handbook of Biometrics, New York: Springer, 2007.

[5] K. B. Kamal Hajari, "A Review of Issues and Challenges in Designing Iris Recognition Systems for Noisy Imaging Environment," Nagpur, India, 2015.

[6] M. A. S. Hina U. Keval, "Can we ID from CCTV: image quality in digital CCTV and face," Dept. of Computer Science, University College London, Gower St, London WC1E 6BT, UK.

[7] S. K. N. M. J. P. D. Dr. S. B. Thorat, "Facial Recognition Technology: An analysis with scope in India," vol. 8, 2010.

[8] L. D. H. A. B. L. A. Jay Goldstein, "Identification of Human Faces," vol. 59, May 1971.

[9] T. Kanade, "Picture Processing System By Computer Complex And Recognition Of Human Faces," Kyota University, November 1973.

[10] M. K. a. L. Sirovich, "Application of the Karhunen-Loeve Procedure for the Characterization of Human Faces," vol. 12, January 1990.

[11] F. R. U. Eigenfaces, "Matthew A. Turk and Alex P. Pentland," 1991.

[12] S. K. B. a. K. Rahul, "Face Recognition by Linear Discriminant Analysis," Jharkhand, India.

[13] A. H. M. P. Timo Ahonen, "Face Detection with Local Binary Patterns: Application to Face Recognition," vol. 28, December 2006.

[14] P. H. T. P. Bernd Heisele, "Face Recognition with Support Vector Machines: Global versus Component-based Approach," 2001. 
[15] B. B. M. Divyarajsinh N. Parmar, "Face Recognition Methods \& Applications," vol. 4, no. 84-86, India.

[16] P. N. Belhumeur, "Ongoing Challenges in Face Recognition," New York.

[17] S. Z. L. Y. W. Haitao Wang, "Face Recognition under Varying Lightning Conditions Using self Quotient Image," Beijing, China, 2004.

[18] K. C. Y. a. R. Cipolla, "Dtection of Human Faces under Scale, Orientation and Viewpoint Variations," Cmbridge, England.

[19] G. M. B. L. J. S. R. N. J. V. B. J. Boom, "The Effect of Image Resolution on Performance of a Face Recognition System," 2006.

[20] S. S. N. R. a. D. W. J. Haibin Ling, "A Study of Face Recognition as People Age," 2007.

[21] R. C. Narayanan Ramanathan, "Face Verification Across Age Progression," vol. 15, November 2006.

[22] H. K. E. a. R. Stiefelhagen, "Why is Facial Occlusion a Challenging Problem?," Computer Science Department, University Karlsruhe, Germany.

[23] J. P. S. a. A. D. Anuse, "Face Recognition under Pose Variations," vol. 5(3), 2014.

[24] V. B. a. T. Vetter, "Face Recognition Based on Fitting a 3D Morphable Model," IEEE Transactions on Pattern Analysis and Machine Intelligence, vol. 25, no. 9, September 2003.

[25] B. P. W. S. a. T. B. Brian Heflin, "Single Image Deblurring for a Real-Time Face Recognition System," 2010.

[26] H. H. A. K. J. Di Wen, "Face Spoof Detection with Image Distortion Analysis," 2015.

[27] A. E.-B. a. A. E.-H. A. S. Tolba, "Face Recognition: A Literature Review," vol. 2, 2006.

[28] "AT\&T Laboratories Cambridge," [Online]. Available: http://www.cl.cam.ac.uk/research/dtg/attarchive/facedatabase.html.

[29] "The Facial Recognition Technology (FERET) Database," [Online]. Available: http://www.itl.nist.gov/iad/humanid/feret/feret_master.html. 
[30] S. B. M. B. Terence Sim, "The CMU Pose, Illumination and Expression Database," vol. 25, December 2003.

[31] "Yale Face Database," [Online]. Available:

http://cvc.cs.yale.edu/cvc/projects/yalefaces/yalefaces.html.

[32] D. K. a. P. B. A. Georghiades, "From few to many: Generative models for recognition under variable pose and illumination," vol. 23, 2001.

[33] J. M. J. K. J. L. G. M. K. Messer, "XM2VTSDB: The Extended M2VTS Database," Washington D. C, 1999.

[34] T. V. Volker Blanz, "A Morphable Model for the Synthesis of 3D Faces," Germany.

[35] R. C. P. J. P. a. A. R. W. Zhao, "Face Recognition: A Literature Survey," vol. 35, December 2003.

[36] V. K. a. R. C. Shaohua Zhou, "Probabilistic recognition of human faces from video," Computer Vision and Image Understanding, vol. 91, pp. 214-245, 2003.

[37] D. S. Varsha Gupta, "A Study of Various Face Detection Methods," International Journel of Advanced Research in Computer and Communication Engineering, vol. 3, no. 5, May 2014.

[38] v. M. a. A. Ross, "A Texture-based Approach to Face Detection".

[39] M. P. a. T. M. Timo Ojala, "Multiresolution Gray Scale and Rotation Invariant Texture CLassification with Local Binary Patterns," IEEE Transactions on Pattern Analysis and Machine Intelligence, vol. 24, no. 7, pp. 971-987, 2002.

[40] M. J. J. Paul Viola, "Robust Real-Time Face Detection," vol. 57, July 112003.

[41] G. Guo, "Face Detection and Recognition," 11 September 2014. [Online]. Available: http://www.csee.wvu.edu/ gdguo/courses/fa14/biom426/Slides/Lecture9.pdf.

[42] X. H. J. D. Cai, "Semi-supervised discriminant analysis," vol. 14, 2007.

[43] K. M. G. a. P. L. Delac, "Appearance based statistical methods for face recognition," Zadar, Croatia, 2005.

[44] O. a. A. A. Toygar, "Face recognition using PCA, LDA and ICA approaches on colored images," vol. 3.1, no. 735-743, 2003. 
[45] P. Comon, "Independent component analysis, A new concept?," Vols. 36 (1994) 287-314, August 1992.

[46] H. J. S. Vaseghi, "Principal and Independent Component Analysis in Image Processing," Brunel University, Department of Electronics and Computer Engineering.

[47] S. B. e. a. Gokturk, "Model-based face tracking for view-independent facial expression recognition," 2002.

[48] G. J. E. a. C. J. T. Timothy F. Cootes, "Active Appearance Models," IEEE Transactions on Pattern Analysis and Machine Intelligence, vol. 23, no. 6, June 2001.

[49] J.-M. F. N. K. a. C. Laurenz Wiskott, "Face Recognition by Elastic Bunch Graph Matching," 1997.

[50] H. H. a. A. K. J. Koichiro Niinuma, "Automatic Multi-view Face Recognition via 3D Model Based Pose Regularization," East Lansing, MI, U.S.A.

[51] P. G. P. J. P. a. T. V. Volker Blanz, "Face Recognition Based on Frontal Views Generated from NonFrontal Images," Gaithersburg, MD, USA.

[52] M. a. A. H. Pietikainen, Texture Features in Facial Image Analysis, Springer Berlin Heidelberg, 2005.

[53] J. S. a. A. F. B. Amos Y. Johnson, "Predicting large population data cumulative match characteristic performance from small population data," Guildford, UK, 2003.

[54] C. L. a. W. Harrell, "Completing the 3-dimensional picture," American journal of orthodontics and dentofacial orthopedics, May 2008.

[55] "American National Standard for Information Systems-Data Format for the Interchange of Fingerprint Facial, \& Other Biometric Information - Part I," National Institute of Standards and Technology, 2007.

[56] R. M. D. Euclides N. Arcoverde, "Real-Time Head Pose Estimation for Mobile Devices," Integrated Computer Aided Engineering, 2014.

[57] NeuroTechnology, "NeuroTechnology MegaMatcher 5.0, VeriFinger7.0, VeriLook 5.5, VeriEye 2.8 and VeriSpeak 2.1 SDK," November 2014. [Online]. Available: http://www.neurotechnology.com/download.html. 
[58] "Cognitec- Face Recognition Company," November 2011. [Online]. Available: http://www.planetbiometrics.com/creo_files/upload/article-files/Cognitec-Brochure-1-11-2011Web.pdf.

[59] Y. V. V. Y. I. K. Vladimir A. Knyaz, "Technique for real-time frontal face image acquisition using stereo system," 2013.

[60] V. B. a. T. Vetter, "Face Recognition Based on Fittinf a 3D Morphable Model," IEEE Transactions on Pattern Analysis and Machine Intelligence, vol. 25, no. 9, September 2003.

[61] T. C. a. C. T. G.J. Edwards, "Face Recognition Using Active Appearance Models," in 5th European Conference on Computer Vision, Londaon, UK, 1998. 